\title{
The New $(g-2)$ Experiment:
}

A Proposal to Measure the Muon Anomalous

Magnetic Moment to \pm 0.14 ppm Precision

Submitted to FNAL

February 9, 2009

New $(g-2)$ Collaboration 


\section{The New $(g-2)$ Experiment:}

\section{A Proposal to Measure the Muon Anomalous Magnetic Moment to \pm 0.14 ppm Precision}

New $(g-2)$ Collaboration: R.M. Carey ${ }^{1}$, K.R. Lynch ${ }^{1}$, J.P. Miller ${ }^{1}$, B.L. Roberts ${ }^{1}$, W.M. Morse', Y.K. Semertzidis' , V.P. Druzhinin ${ }^{3}$, B.I. Khazin ${ }^{3}$, I.A. Koop ${ }^{3}$, I. Logashenko ${ }^{3}$, S.I. Redin ${ }^{3}$, Y.M. Shatunov ${ }^{3}$, Y. Orlov ${ }^{4}$, R.M. Talman ${ }^{4}$, B. Casey $^{5}$, J. Johnstone ${ }^{5}$, D. Harding ${ }^{5}$, A. Klebaner ${ }^{5}$, A. Leveling ${ }^{5}$, J-F. Ostiguy ${ }^{5}$, N. Mokhov ${ }^{5}$, D. Neuffer ${ }^{5}$, M. Popovic ${ }^{5}$, S. Strigonov ${ }^{5}$, M. Syphers ${ }^{5}$, G. Velev $^{5}$, S. Werkema ${ }^{5}$, F. Happacher ${ }^{6}$, G. Venanzoni ${ }^{6}$, P. Debevec $^{7}$, M. Grosse-Perdekamp ${ }^{7}$, D.W. Hertzog ${ }^{7}$, P. Kammel ${ }^{7}$, C. Polly ${ }^{7}$, K.L. Giovanetti ${ }^{8}$, K. Jungmann ${ }^{9}$, C.J.G. Onderwater ${ }^{9}$, N. Saito ${ }^{10}$, C. Crawford ${ }^{11}$, R. Fatemill, T.P. Gorringe ${ }^{11}$, W. Korsch ${ }^{11}$, B. Plaster ${ }^{11}$, V. Tishchenko ${ }^{11}$, D. Kawall ${ }^{12}$, T. Chupp ${ }^{13}$, C. Ankenbrandt ${ }^{14}$, M.A Cummings ${ }^{14}$, R.P. Johnson ${ }^{14}$, C. Yoshikawa ${ }^{14}$, André de Gouvêa ${ }^{15}$, T. Itahashi ${ }^{16}$, Y. Kuno ${ }^{16}$, G.D. Alkhazov ${ }^{17}$, V.L. Golovtsov' ${ }^{17}$, P.V. Neustroev ${ }^{17}$, L.N. Uvarov ${ }^{17}$, A.A. Vasilyev ${ }^{17}$, A.A. Vorobyov ${ }^{17}$, M.B. Zhalov ${ }^{17}$, F. Gray ${ }^{18}$, D. Stöckinger ${ }^{19}$, S. Baeßler ${ }^{20}$, M. Bychkov ${ }^{20}$, E. Frlež ${ }^{20}$, and D. Počanićc ${ }^{20}$

${ }^{1}$ Department of Physics, Boston University, Boston, MA 02215, USA

${ }^{2}$ Brookhaven National Laboratory, Upton, NY 11973, USA ${ }^{3}$ Budker Institute of Nuclear Physics, 630090 Novosibirsk, Russia

${ }^{4}$ Newman Laboratory, Cornell University, Ithaca, NY 14853, USA

${ }^{5}$ Fermi National Accelerator Laboratory, Batavia, IL 60510, USA

${ }^{6}$ Laboratori Nazionali di Frascati, INFN, I-00044 Frascati (Rome), Italy

${ }^{7}$ Department of Physics, University of Illinois, Urbana-Champaign, IL 61801, USA

${ }^{8}$ Department of Physics, James Madison University, Harrisonburg, VA 22807, USA

${ }^{9}$ Kernfysisch Versneller Instituut, Rijksuniversiteit, Groningen, NL 9747 AA Groningen, The Netherlands

${ }^{10}$ KEK, High Energy Accelerator Research Organization, Tsukuba, Ibaraki 305-0801, Japan

${ }^{11}$ Department of Physics, University of Kentucky, Lexington, Ky 40506, USA 
${ }^{12}$ Department of Physics, University of Massachusetts, Amherst, MA 01003, USA

${ }^{13}$ Physics Department, University of Michigan, Ann Arbor, MI 48109, USA

$$
{ }^{14} \text { Muons, Inc., Batavia, IL 60510, USA }
$$

${ }^{15}$ Physics \& Astronomy Department, Northwestern University, Evanston, IL 60208, USA

${ }^{16}$ Department of Physics, Osaka University, Toyonaka, Osaka 560-0043, Japan

${ }^{17}$ Petersburg Nuclear Physics Institute, Gatchina 188350, Russia

${ }^{18}$ Department of Physics and Computational

Science, Regis University, Denver, CO 80221, USA

${ }^{19}$ Technische Universität Dresden, Institut für Kern-

und Teilchenphysik, D-01062 Dresden, Germany

${ }^{20}$ Department of Physics, The University of Virginia, Charlottesville, VA 22904, USA

\section{Request: $4 \times 10^{20}$ protons on target in 6 of 20 Booster batches during $15 \mathrm{~Hz}$ operation \\ February 9, 2009}

Contactpersons: David W. Hertzog (hertzog@illinois.edu, 217-333-3988)

B. Lee Roberts (roberts@bu.edu, 617-353-2187) 


\begin{abstract}
We propose to measure the muon anomalous magnetic moment, $a_{\mu}$, to $0.14 \mathrm{ppm}-\mathrm{a}$ fourfold improvement over the $0.54 \mathrm{ppm}$ precision obtained in the BNL experiment E821. The muon anomaly is a fundamental quantity and its precise determination will have lasting value. The current measurement was statistics limited, suggesting that greater precision can be obtained in a higher-rate, next-generation experiment. We outline a plan to use the unique FNAL complex of proton accelerators and rings to produce high-intensity bunches of muons, which will be directed into the relocated BNL muon storage ring. The physics goal of our experiment is a precision on the muon anomaly of $16 \times 10^{-11}$, which will require 21 times the statistics of the BNL measurement, as well a factor of 3 reduction in the overall systematic error. Our goal is well matched to anticipated advances in the worldwide effort to determine the standard model (SM) value of the anomaly. The present comparison, $\Delta a_{\mu}($ Expt. $-\mathrm{SM})=(295 \pm 81) \times 10^{-11}$, is already suggestive of possible new physics contributions to the muon anomaly. Assuming that the current theory error of $51 \times 10^{-11}$ is reduced to $30 \times 10^{-11}$ on the time scale of the completion of our experiment, a future $\Delta a_{\mu}$ comparison would have a combined uncertainty of $\approx 34 \times 10^{-11}$, which will be a sensitive and complementary benchmark for proposed standard model extensions. The experimental data will also be used to improve the muon. EDM limit by up to a factor of 100 and make a higher-precision test of Lorentz and CPT violation.

We describe in this Proposal why the FNAL complex provides a uniquely ideal facility for a next-generation $(g-2)$ experiment. The experiment is compatible with the fixed-target neutrino program; indeed, it requires only the unused Booster batch cycles and can acquire the desired statistics in less than two years of running. The proton beam preparations are largely aligned with the new Mu2e experimental requirements. The $(g-2)$ experiment itself is based on the solid foundation of E821 at BNL, with modest improvements related to systematic error control. We outline the motivation, conceptual plans, and details of the tasks, anticipated budget, and timeline in this proposal.
\end{abstract}




\section{Contents}

$\begin{array}{ll}\text { I. Executive Summary } & 7\end{array}$

II. Introduction 9

A. Principle of the Experiment 11

B. Experimental Specifics 14

III. The Physics Case for a New $(g-2)$ Experiment 16

A. The Standard-Model Value of $a_{\mu} \quad 18$

1. QED and Weak Contributions 18

2. The Hadronic Contribution 19

3. The Lowest-order Hadronic Contribution 21

4. $\mathrm{BaBar} e^{+} e^{-} \rightarrow \pi^{+} \pi^{-}$Data 23

5. $a_{\mu}^{\text {had;LO }}$ from Hadronic $\tau$ decay 23

6. The Hadronic Light-by-Light Contribution 24

B. Summary of the Standard Model Value and Comparison with Experiment 24

1. $R(s)$ Measurements and the Higgs Mass, $M_{H} \quad 26$

C. Expected Improvements in the Standard Model Value 27

D. Physics Beyond the Standard Model 28

1. $a_{\mu}$ as a benchmark for models of new physics 31

2. $a_{\mu}$ is sensitive to quantities that are difficult to measure at the LHC 33

IV. A New $(g-2)$ Experiment $\quad 37$

A. Scientific Goal $\quad 37$

B. Key Elements to a New Experiment 37

C. The Expected Flash at FNAL 39

D. Event Rate and POT Request Calculation 40

V. Beam Preparation 43

A. Meeting the Experimental Requirements 44

B. Bunch Formation 46

C. Beam Delivery and Transfer 48

D. Target Station, Pion Capture and Decay $\quad 50$ 
F. Toward a Beam Rate Calculation 50

F. Experimental Facility 51

G. Environmental Assessment 52

H. Opening the Inflector Ends

I. Accelerator R\&D

J. Muon Storage Ring Magnet

K. Relocating the Storage Ring to FNAL

VI. The Precision Magnetic Field $\quad 59$

A. Methods and Techniques 59

B. Past improvements 61

C. Shimming the Storage Ring Magnetic Field 63

D. Further Improvements $\quad 66$

VII. $\omega_{a}$ Measurement $\quad 69$

$\begin{array}{ll}\text { A. Overview } & 69\end{array}$

B. Electromagnetic Calorimeters $\quad 73$

$\begin{array}{ll}\text { C. Position-Sensitive Detectors } & 74\end{array}$

D. Waveform Digitizers for Calorimeter Readout $\quad 75$

E. Clock systems 78

F. Data Acquisition $\quad 79$

VIII. Systematic uncertainties on $\omega_{a} \quad 81$

A. Gain Changes and Energy-Scale Stability 82

B. Lost Muons 83

C. Pileup 84

D. Coherent Betatron Oscillations 86

E. Electric Field and Pitch Correction 87

F. $\omega_{0}$ Systematic Uncertainty Summary 88

IX. Parasitic Measurement of the Muon Electric Dipole Moment 88

A. E821 Traceback System 90

B. Improved Traceback System 91 
X. Manpower, Cost Estimate and Schedule 93

A. Manpower 93

B. Cost Estimate 93

$\begin{array}{ll}\text { C. Schedule } & 97\end{array}$

$\begin{array}{ll}\text { XI. Planned R\&D Efforts } & 98\end{array}$

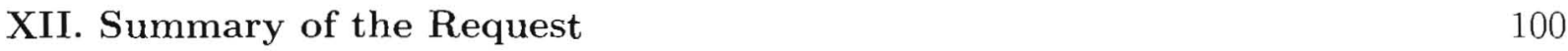

References 101

A. Muon Collection and Transport Beamline 106

1. Beamline Overview 106

2. Pion production 108

3. Transport in AP2 109

4. Performance Estimate 110

B. Beam Dynamics and Scraping 112

1. The Kicker and Quadrupoles 112

2. Beam Dynamics in the Ring 112

3. Oscillating Dipole Method of Scraping 115

4. Pulsed Octupole Method to Remove the CBO 117

$\begin{array}{ll}\text { C. New Calorimeters } & 121\end{array}$ 


\section{EXECUTIVE SUMMARY}

The muon anomalous magnetic moment $a_{\mu}$ is a low-energy observable, which can be both measured and computed to high precision. The comparison between experiment and the standard model (SM) is a sensitive test of new physics. At present, both measurement and theory cite similar sub-ppm uncertainties, and the " $(g-2)$ test" is being used to constrain standard model extensions. Indeed, the difference, $\Delta a_{\mu}($ Expt $-\mathrm{SM})=(295 \pm 81) \times 10^{-11}$, is a highly cited result, and a possible harbinger of new TeV-scale physics. The present proposal is to reduce the experimental uncertainty to create a more precise $(g-2)$ test that will sharply discriminate among models. A significant effort is ongoing worldwide to reduce the uncertainty on the SM contributions. During the time required to mount, run and analyze the New $(g-2)$ Experiment, we expect that the theory uncertainty will have improved considerably.

The experimental determination of the muon anomalous magnetic moment $a_{\mu}$ has an uncertainty of $0.54 \mathrm{ppm}$, which is dominated by the statistical error of $0.46 \mathrm{ppm}$. This suggests that a further increase in precision is possible if a higher integrated number of stored muons can be obtained. We propose to measure $a_{\mu}$ at FNAL to an uncertainty of $0.14 \mathrm{ppm}$, derived from a $0.10 \mathrm{ppm}$ statistical sample and roughly equal $0.07 \mathrm{ppm}$ systematic uncertainty contributions from measurement of the magnetic field and from measurement of the precession frequency. Twenty-one times more events are required compared to the E821 experiment, which completed its data taking in 2001. Our proposal efficiently uses the FNAL beam complex - in parasitic mode to the high-energy neutrino program-- to produce the necessary flux of muons, which will be injected and stored in the relocated muon storage ring. In less than two years of running, the statistical goal can be achieved for positive muons. A follow-up run using negative muons is possible, depending on future scientific motivation. Two additional physics results will be obtained from the same data: a new limit on the muon's electric dipole moment; and, a more stringent limit on possible CPT or Lorentz violation in muon spin precession.

The beam concept at FNAL can deliver the required flux at a higher injection frequency compared to BNL. With the long FNAL decay channel, the stored muon rate per proton rises considerably, while largely eliminating the troubling hadronic-induced beam-injection background that plagued the BNL measurement. The new experiment will require upgrades 
and maintenance on several components of the storage ring and a new suite of segmented electromagnetic calorimeters and position-sensitive detectors. A modern data acquisition system will be used to read out waveform digitizer data and store it so that both the traditional event mode and a new integrating mode of data analysis can both be used in parallel. Improvements in the field-measuring system are also required. They will both follow well-developed upgrade plans and address challenges for even further precision through a program of R\&D efforts.

A challenging aspect of the new experiment will be the disassembly, transport, and reassembly of the BNL storage ring (See Fig. 1). We have examined the tasks in consultation with the lead project engineers and can estimate the time and cost required with a fair degree of confidence. The ring will be placed in a new and relatively modest building at FNAL at the end of the AP2 line (near the AP0 blockhouse). The critical timescale to be ready for data taking is driven by the effort to relocate the ring to FNAL and to reshim it to very high field uniformity. Detector and software development will take place concurrently. The Laboratory cost for 1) the ring relocation; 2) special beamline elements; and 3) detector, electronics and DAQ systems, is estimated to be approximately $\$ 20 \mathrm{M}$. The Collaboration is built from a core group of E821 participants, together with many new domestic and international groups.

Our planning envisions a development period following scientific approval of approximately 1 year, during which time we would complete a detailed design and cost document. During this time period, we will complete the plans to move the storage ring, finalize the beam optics, site and design the building, and begin R\&D on the detectors, electronics, and field-measuring systems. These tasks mainly involve personnel, and do not incur large capital costs. We note that many of the beam developments required are also needed for the approved Mu2e experiment. 


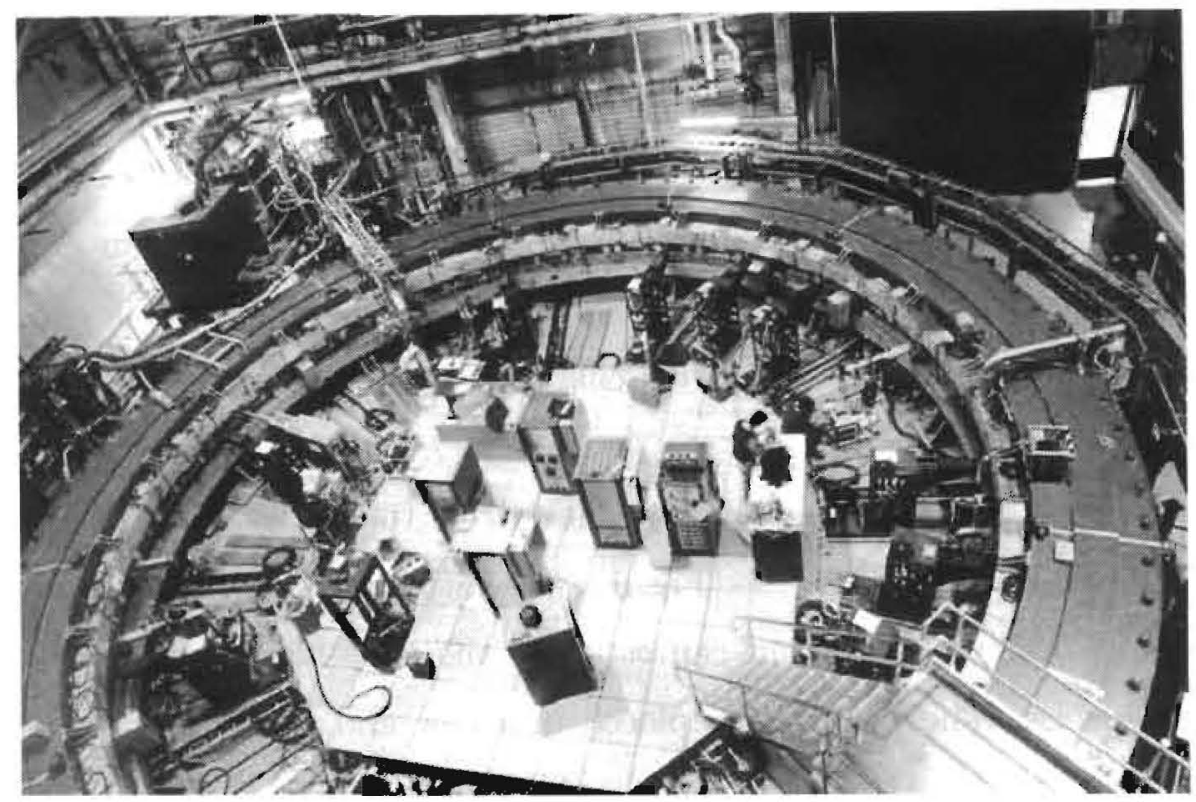

FIG. 1: The existing muon storage ring that will be relocated to FNAL for the New $(g-2)$ Experiment.

\section{INTRODUCTION}

The muon magnetic moment is related to its intrinsic spin by the gyromagnetic ratio $g_{\mu}$ :

$$
\vec{\mu}_{\mu}=g_{\mu}\left(\frac{q}{2 m}\right) \vec{S}
$$

where $g_{\mu}=2$ is expected for a structureless, spin- $\frac{1}{2}$ particle of mass $m$ and charge $q= \pm|e|$, with radiative corrections ( $\mathrm{RC}$ ), which couple the muon spin to virtual fields, introduce an anomalous magnetic moment defined by

$$
a_{\mu}=\frac{1}{2}\left(g_{\mu}-2\right)
$$

The leading $\mathrm{RC}$ is the lowest-order (LO) quantum electrodynamic process involving the exchange of a virtual photon, the "Schwinger term," [1] giving $a_{\mu}(\mathrm{QED} ; \mathrm{LO})=\alpha / 2 \pi \approx$ $1.16 \times 10^{-3}$. The complete standard model value of $a_{\mu}$, currently evaluated to a precision of approximately $0.5 \mathrm{ppm}$ (parts per million), includes this first-order term along with higherorder QED processes, electroweak loops, hadronic vacuum polarization, and other higherorder hadronic loops. The measurement of $a_{\mu}$ in Brookhaven E821 was carried out to a similar precision [2]. The difference between experimental and theoretical values for $a_{\mu}$ is a 
valuable test of the completeness of the standard model. At sub-ppm precision, such a test explores TeV-scale physics. The present difference between experiment and theory is

$$
\left.\Delta a_{\mu}(\operatorname{Expt}-\mathrm{SM})=(295 \pm 81)\right) \times 10^{-11},(3.6 \sigma)
$$

which is based on the 2008 summary of the standard model (SM) by de Rafael [3]. A contribution to the muon anomaly of this magnitude is expected in many popular standard model extensions, while other models predict smaller or negligible effects. In the LHC era, accurate and precise low-energy observables, such as $a_{\mu}$, will help distinguish between candidate theories in defining a new standard model. The motivation for a new, more precise $(g-2)$ experiment, is to contribute significantly to the determination of the expected new physics at the electroweak scale. We devote a chapter of this proposal to the present and expected future status of the standard model evaluation and to the physics probed by an improved measurement.

Precision measurements of $a_{\mu}$ have a rich history dating nearly 50 years. In Table I we give a brief summary. With improved experimental methods, the precision on the measurement of $a_{\mu}$ has increased considerably. Advances in theoretical techniques-often driven by the promise of a new more precise measurement have largely stayed at pace and we expect that the approval of a new FNAL based experiment will continue to drive improvements in the determination of the SM value in the future.

We propose to measure the muon $(g-2)$ to the limit of the present experimental technique, which will require more than 20 times the current event statistics. While we will largely follow the proven method pioneered at CERN and significantly improved at Brookhaven, the new experiment requires the unique high-intensity proton accelerator complex at Fermilab to obtain a 21-times larger statistical sample. Upgrades in detectors, electronics, and field-measuring equipment will be required as part of a comprehensive plan to reduce systematic errors. These tasks are relatively well known to us as the collaboration is quite experienced in the proposed measurement. Subsequent chapters will outline the main issues and the beam use plan that is aimed to complete the experiment in less than 2 years of running. 
TABLE I: Summary of $a_{\mu}$ results from CERN and BNL, showing the evolution of experimental precision over time. The average is obtained from the BNL 1999, 2000 and 2001 data sets only.

\begin{tabular}{ccccccc}
\hline \hline Experiment & Years & Polarity & $a_{\mu} \times 10^{10}$ & Precision [ppm] & Reference \\
\hline CERN I & 1961 & $\mu^{+}$ & $11620000(50000)$ & 4300 & {$[4]$} \\
CERN II & $1962-1968$ & $\mu^{+}$ & $11661600(3100)$ & 270 & {$[5]$} \\
CERN III & $1974-1976$ & $\mu^{+}$ & $11659100(110)$ & 10 & {$[7]$} \\
CERN III & $1975-1976$ & $\mu^{-}$ & $11659360(120)$ & 10 & {$[7]$} \\
BNL & 1997 & $\mu^{+}$ & $11659251(150)$ & 13 & {$[8]$} \\
BNL & 1998 & $\mu^{+}$ & $11659191(59)$ & 5 & {$[9]$} \\
BNL & 1999 & $\mu^{+}$ & $11659202(15)$ & 1.3 & {$[10]$} \\
BNL & 2000 & $\mu^{+}$ & $11659204(9)$ & 0.73 & {$[11]$} \\
BNL & 2001 & $\mu^{-}$ & $11659214(9)$ & 0.72 & {$[12]$} \\
\hline Average & & & $11659208.0(6.3)$ & 0.54 & {$[2]$} \\
\hline \hline
\end{tabular}

\section{A. Principle of the Experiment}

The cyclotron $\omega_{c}$ and spin precession $\omega_{s}$ frequencies for a muon moving in the horizontal plane of a magnetic storage ring are given by:

$$
\vec{\omega}_{c}=-\frac{q \vec{B}}{m \gamma}, \quad \vec{\omega}_{s}=-\frac{g q \vec{B}}{2 m}-(1-\gamma) \frac{q \vec{B}}{\gamma m} .
$$

The anomalous precession frequency $\omega_{a}$ is determined from the difference

$$
\vec{\omega}_{a}=\vec{\omega}_{s}-\vec{\omega}_{c}=-\left(\frac{g-2}{2}\right) \frac{q \vec{B}}{m}=-a_{\mu} \frac{q \vec{B}}{m} .
$$

Because electric quadrupoles are used to provide vertical focusing in the storage ring, their electric field is seen in the muon rest frame as a motional magnetic field that can affect the spin precession frequency. In the presence of both $\vec{E}$ and $\vec{B}$ fields, and in the case that $\vec{\beta}$ is perpendicular to both $\vec{E}$ and $\vec{B}$, the expression for the anomalous precession frequency becomes

$$
\vec{\omega}_{a}=-\frac{q}{m}\left[a_{\mu} \vec{B}-\left(a_{\mu}-\frac{1}{\gamma^{2}-1}\right) \frac{\vec{\beta} \times \vec{E}}{c}\right] .
$$


The coefficient of the $\vec{\beta} \times \vec{E}$ term vanishes at the "magic" momentum of $3.094 \mathrm{GeV} / c$, where $\gamma=29.3$. Thus $a_{\mu}$ can be determined by a precision measurement of $\omega_{a}$ and $B$. At this magic momentum, the electric field is used only for muon storage and the magnetic field alone determines the precession frequency. The finite spread in beam momentum and vertical betatron oscillations introduce small (sub ppm) corrections to the precession frequency. These are the only corrections made to the measurement.

The longitudinally polarized muons, which are injected into the storage ring at the magic momentum, have a time-dilated muon lifetime of $64.4 \mu \mathrm{s}$. A measurement period of typically $700 \mu$ s follows each injection or "fill." The net spin precession depends on the integrated field seen by a muon along its trajectory. The magnetic field used in Eq. 6 refers to an average over muon trajectories during the course of the experiment. The trajectories of the muons must be weighted with the magnetic field distribution. To minimize the precision with which the average particle trajectories must be known, the field should be made as uniform as possible.

Because of parity violation in the weak decay of the muon, a correlation exists between the muon spin and decay electron direction. This correlation allows the spin direction to be measured as a function of time. In the rest frame of the muon-indicated by starred quantities the differential probability for the electron to emerge with a normalized energy $y=E^{*} / E_{\max }\left(E_{\max }=52.8 \mathrm{MeV}\right)$ at an angle $\theta^{*}$ with respect to the muon spin is

$$
\begin{aligned}
\frac{d P\left(y, \theta^{*}\right)}{d y d \Omega}=(1 / 2 \pi) n^{*}(y)\left[1-\alpha^{*}(y) \cos \theta^{*}\right] & \text { with } \\
n^{*}(y) & =y^{2}(3-2 y) \text { and } \\
\alpha^{*}(y) & =\frac{q}{e} \frac{2 y-1}{3-2 y} .
\end{aligned}
$$

Figure 2a shows the quantities $n^{*}(y)$ and $\alpha^{*}(y)$. Electrons with $y<0.5$ are emitted preferentially along the (negative) muon spin direction and those with $y>0.5$ are more likely emitted opposite to the spin. Because both $n^{*}$ and $\alpha^{*}$ are larger for $y>0.5$, decay electrons tend to emerge in the direction opposite to the muon spin. Like the muon spin, the angular distribution of the electrons in the muon rest frame rotates at the angular frequency $\omega_{a}$. Figure $2 \mathrm{~b}$ shows the same differential quantities in the boosted laboratory frame $\left(n^{*} \rightarrow N, \alpha^{*} \rightarrow A\right)$. Here, $E_{\text {max }} \approx 3.1 \mathrm{GeV}$ and $A$ is the laboratory asymmetry. The statistical uncertainty on the measurement of $\omega_{a}$ is inversely proportional to the ensemble-averaged figure-of-merit (FOM) $N A^{2}$. The differential quantity $N A^{2}$, shown in the Fig. $2 \mathrm{~b}$, illustrates the relative 


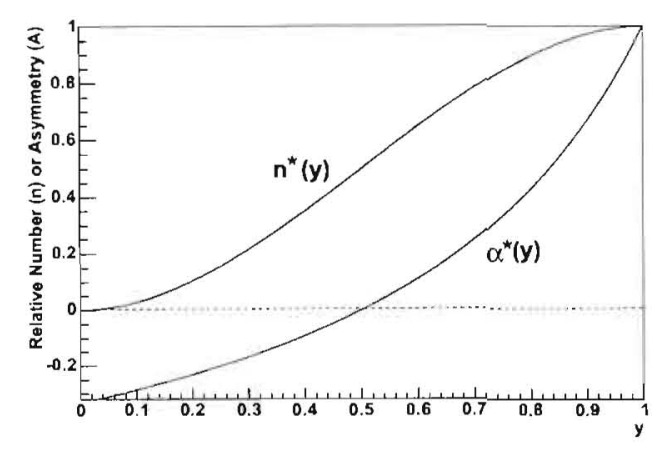

(a) Center-of-mass frame

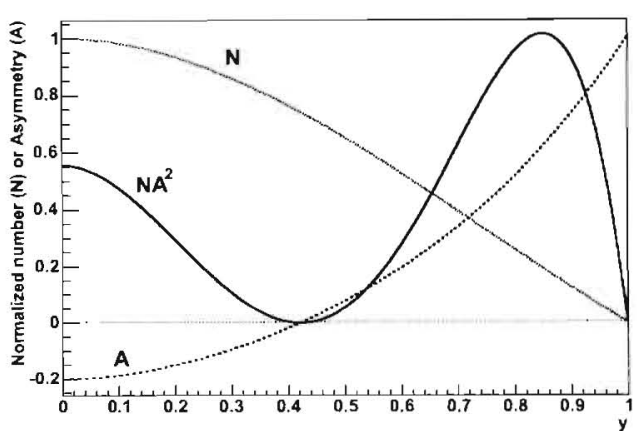

(b)Lab frame

FIG. 2: Relative number and asymmetry distributions versus electron fractional energy $y$ in the muon rest frame (left panel) and in the laboratory frame (right panel). The differential figure-ofmerit product $N A^{2}$ in the laboratory frame illustrates the importance of the higher-energy electrons in reducing the measurement statistical uncertainty.

weight by electron energy to the ensemble average FOM.

Because the stored muons are highly relativistic, the decay angles observed in the laboratory frame are greatly compressed into the direction of the muon momenta. The lab energy of the relativistic electrons is given by

$$
E_{l a b}=\gamma\left(E^{*}+\beta p^{*} c \cos \theta^{*}\right) \approx \gamma E^{*}\left(1+\cos \theta^{*}\right) .
$$

Because the laboratory energy depends strongly on the decay angle $\theta^{*}$, setting a laboratory threshold $E_{t h}$ selects a range of angles in the muon rest frame. Consequently, the integrated number of electrons above $E_{t h}$ is modulated at frequency $\omega_{a}$ with a threshold-dependent asymmetry. The integrated decay electron distribution in the lab frame has the form

$$
N_{\text {ideal }}(t)=N_{0} \exp \left(-t / \gamma \tau_{\mu}\right)\left[1-A \cos \left(\omega_{a} t+\phi\right)\right]
$$

where $N_{0}, A$ and $\phi$ are all implicitly dependent on $E_{t h}$. For a threshold energy of $1.8 \mathrm{GeV}$ $(y \approx 0.58$ in Fig. $2 \mathrm{~b}$ ), the asymmetry is $\approx 0.4$ and the average FOM is maximized. A representative electron decay time histogram is shown in Fig. 3.

To determine $a_{\mu}$, we divide $\omega_{a}$ by $\widetilde{\omega}_{p}$, where $\widetilde{\omega}_{p}$ is the measure of the average magnetic field seen by the muons. The magnetic field, measured using NMR, is proportional to the free proton precession frequency, $\omega_{p}$. 


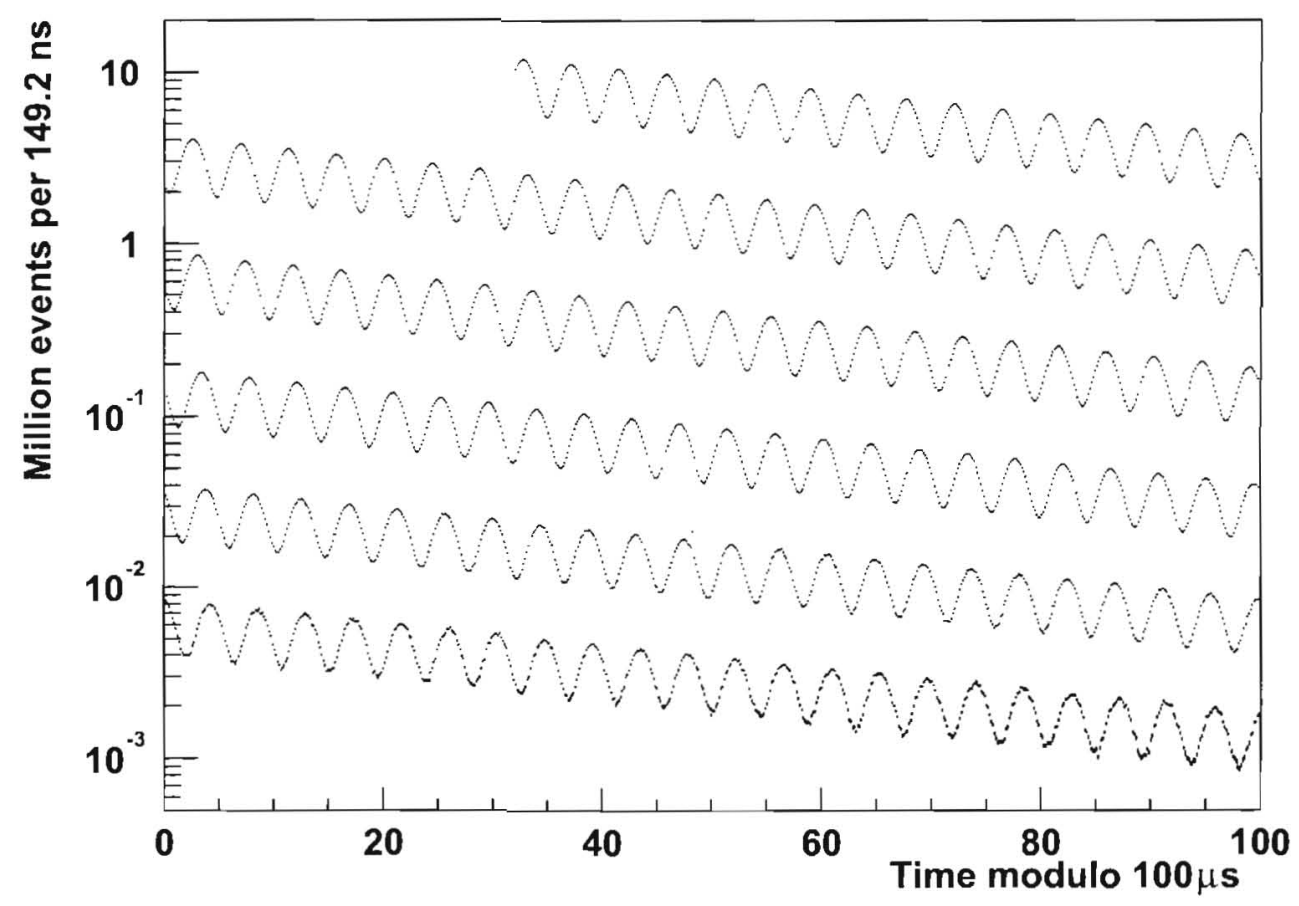

FIG. 3: Distribution of electron counts versus time for the 3.6 billion muon decays. The data is wrapped around modulo $100 \mu \mathrm{s}$.

The muon anomaly is given by:

$$
a_{\mu}=\frac{\omega_{a}}{\omega_{L}-\omega_{a}}=\frac{\omega_{a} / \tilde{\omega}_{p}}{\omega_{L} / \widetilde{\omega}_{p}-\omega_{a} / \widetilde{\omega}_{p}}=\frac{\mathcal{R}}{\lambda-\mathcal{R}}
$$

where $\omega_{L}$ is the Larmor precession frequency of the muon. The ratio $\mathcal{R}=\omega_{a} / \tilde{\omega}_{p}$ is measured in our experiment and the muon-to-proton magnetic moment ratio

$$
\lambda=\omega_{L} / \omega_{p}=3.18334539(10)
$$

is determined from muonium hyperfine level structure measurements $[13,14]$.

\section{B. Experimental Specifics}

Equation 12 demonstrates that both $\omega_{a}$ and $\tilde{\omega}_{p}$ must be known to high precision to determine $a_{\mu}$ from the experiment. The magnetic field is measured using NMR techniques. In E821, it was shimmed to an azimuthally averaged uniformity of better than $\pm 1 \mathrm{ppm}$. 
Improvements will be made in the re-shimming process with the aim of an even more uniform field. To monitor the magnetic field during data collection, 366 fixed NMR probes are placed around the ring to track the field in time. A trolley with 17 NMR probes will map the field in the storage ring, in vacuum, several times per week. The trolley probes will be calibrated with a special spherical water probe, which provides a calibration to the free proton spin precession frequency $\omega_{p}$. The details are described later.

The experiment is run using positive muons owing to the higher cross section for $\pi^{+}$ production from $8-\mathrm{GeV}$ protons. In the ring, the decay positrons are detected in new, segmented tungsten-scintillating-fiber calorimeters [15] where their energy and arrival time are measured. The number of high-energy positrons above an energy threshold $E_{t h}$ as a function of time is given by

$$
N(t)=N_{0}\left(E_{t h}\right) e^{-t / \gamma \tau}\left[1+A\left(E_{t h}\right) \sin \left(\omega_{a} t+\phi_{a}\left(E_{t h}\right)\right)\right]
$$

The uncertainty on $\omega_{a}$ is given by

$$
\frac{\delta \omega_{a}}{\omega_{a}}=\frac{\sqrt{2}}{\omega_{a} \tau_{\mu} \sqrt{N} A}
$$

where the energy threshold $E_{t h}$ is chosen to optimize the quantity $N A^{2}$.

The key to any precision measurement is the systematic errors. A summary of the realized systematic errors from BNL E821 is given in Table II. Our goal is to improve the net systematic error on both categories- $-\omega_{a}$ and $\omega_{p}$. to $\approx 0.07 \mathrm{ppm}$, each. The design of the new experiment is based on a full consideration of items in this table, which will be discussed in detail in the proposal. In some cases, $R \& D$ work will be required to develop instrumentation to achieve the stated systematic goals. 


\begin{tabular}{|l|c|c|c||l|c|c|c|}
\hline$\sigma_{\text {syst }} \omega_{p}$ & $\begin{array}{c}1999 \\
(\mathrm{ppm})\end{array}$ & $\begin{array}{c}2000 \\
(\mathrm{ppm})\end{array}$ & $\begin{array}{c}2001 \\
(\mathrm{ppm})\end{array}$ & $\begin{array}{l}\sigma_{\text {syst }} \omega_{a} \\
(\mathrm{ppm})\end{array}$ & $\begin{array}{c}1999 \\
(\mathrm{ppm})\end{array}$ & $\begin{array}{c}2000 \\
(\mathrm{ppm})\end{array}$ \\
\hline Inflector fringe field & 0.20 & - & - & Pile-Up & 0.13 & 0.13 & 0.08 \\
Calib. of trolley probes & 0.20 & 0.15 & 0.09 & AGS background & 0.10 & 0.01 & $\ddagger$ \\
Tracking $B$ with time & 0.15 & 0.10 & 0.07 & Lost muons & 0.10 & 0.10 & 0.09 \\
Measurement of $B_{0}$ & 0.10 & 0.10 & 0.05 & Timing shifts & 0.10 & 0.02 & $\ddagger$ \\
$\mu$-distribution & 0.12 & 0.03 & 0.03 & E-field/pitch & 0.08 & 0.03 & $\ddagger$ \\
Absolute calibration & 0.05 & 0.05 & 0.05 & Fitting/binning & 0.07 & 0.06 & $\ddagger$ \\
Others ${ }^{\dagger}$ & 0.15 & 0.10 & 0.07 & CBO & 0.05 & 0.21 & 0.07 \\
& & & & Beam debunching & 0.04 & 0.04 & $\ddagger$ \\
& & & & Gain changes & 0.02 & 0.13 & 0.12 \\
\hline Total for $\omega_{p}$ & 0.4 & 0.24 & 0.17 & Total for $\omega_{a}$ & 0.3 & 0.31 & 0.21 \\
\hline
\end{tabular}

TABLE II: Systematic Errors from the E821 running periods in 1999, 2000 and 2001 [10-12]. CBO stands for coherent betatron oscillations. The pitch correction comes from the vertical betatron oscillations, since $\vec{\beta} \cdot \vec{B} \neq 0$. The E-field correction is for the radial electric field seen by muons with $p_{\mu} \neq p_{\text {magic. }}$

†Higher multipoles, the trolley frequency, temperature, and voltage response, eddy currents from the kickers, and time-varying stray fields

†In 2001 AGS background, timing shifts, E field and vertical oscillations, beam debunching/randomization, binning and fitting procedure together equaled $0.11 \mathrm{ppm}$

\section{THE PHYSICS CASE FOR A NEW $(g-2)$ EXPERIMENT}

In the first part of this section we present the standard model theory of the muon anomalous magnetic moment (anomaly). Then we discuss physics beyond the standard model that could contribute to the anomaly at a measurable level. The conclusion is that muon $(g-2)$ will play a powerful role in the interpretation of new phenomena that might be discovered at the LHC. If new phenomena are not discovered there, then muon $(g-2)$ becomes even more important, since it would provide one of the few remaining ways to search for new physics at the TeV scale.

The magnetic moment of the muon (or electron), which is aligned with its spin, is given 
by

$$
\vec{\mu}=g \frac{q}{2 m_{\mu, e}} \vec{s}, \quad \underbrace{g=2}_{\text {Dirac }}\left(1+a_{\mu}\right) ;
$$

where the quantity $g$ is exactly 2 in the Dirac theory, $q= \pm e$ with $e$ a positive number. The small number $a$, the anomaly, arises from quantum fluctuations, with the largest contribution coming from the single loop diagram in Fig. 4(a). This contribution was first calculated by Schwinger[1], who obtained $a=(\alpha / 2 \pi)=0.00116 \cdots$. These calculations have been extended to higher powers in $\alpha / \pi$, with the fourth $(\alpha / \pi)^{2}$ and sixth-order $(\alpha / \pi)^{3}$ having been carried out analytically. The eight-order has been evaluated numerically, both for the electron and muon, by Kinoshita and his colleagues, and the tenth-order is presently being evaluated $[16,17]$.

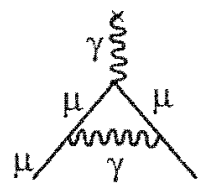

(a)

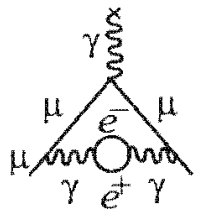

(b)

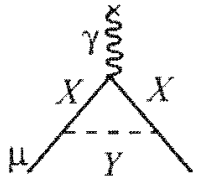

(c)

FIG. 4: The Feynman graphs for: (a) The lowest-order (Schwinger) contribution to the lepton anomaly ; (b) The vacuum polarization contribution, which is one of five fourth-order, $(\alpha / \pi)^{2}$, terms; (c) The schematic contribution of new particles $X$ and $Y$ that couple to the muon.

The electron anomaly is relatively insensitive to heavier physics, so in principle the 0.03 ppb measurement of the electron anomaly [18] should provide a test of QED, but the few ppb precision of the independent measurements of $\alpha$ prevents this comparison. Alternately, one can accept that QED is valid and use the electron anomaly to determine the most precise measurement of $\alpha$, which de Rafael uses in his evaluation[3] of the QED contribution to $a_{\mu}$.

The muon anomaly is an entirely different case. The relative contribution to the muon anomaly of heavier virtual particles goes as $\left(m_{\mu} / m_{e}\right)^{2} \simeq 43,000$, so with much less precision when compared with the electron, the muon anomaly is sensitive to mass scales in the several hundred GeV region. This not only includes the expected contribution of the $W$ and $Z$ bosons, but perhaps contributions from new, as yet undiscovered, particles such as the supersymmetric partners of the electro-weak gauge bosons (see Fig. 4(c)). The contribution from standard-model particles is discussed first, and then the implications for non-standardmodel physics are discussed. 
The standard model value of $a_{\mu}$ has three contributions from radiative processes: QED loops containing leptons $(e, \mu, \tau)$ and photons; loops containing hadrons in vacuum polarization loops where the $e^{+} e^{-}$pair in Fig 4(b) is replaced by hadrons; and weak loops involving the weak gauge bosons $W, Z$, and Higgs such as is shown in Fig. $4(c)$ where $X=W$ and $Y=\nu$, or $X=\mu$ and $Y=Z$. Thus

$$
a_{\mu}(\mathrm{SM})=a_{\mu}(\mathrm{QED})+a_{\mu}(\text { hadronic })+a_{\mu}(\text { weak })
$$

Each of these contributions is discussed below.

\section{A. The Standard-Model Value of $a_{\mu}$}

\section{QED and Weak Contributions}

The QED and electroweak contributions to $a_{\mu}$ are well understood. We take the numerical values from reviews by de Rafael[3, 19] The QED contribution to $a_{\mu}$ has been calculated through four loops, with the leading five loop contributions estimated [16, 17]. The present value is

$$
a_{\mu}^{\mathrm{QED}}=116584718.09(0.02)(0.14)(0.04) \times 10^{-11}
$$

where the uncertainties are from the 4- and 5-loop QED contributions, and the value of $\alpha$ taken from the electron $(g-2)$ value[3].

The electroweak contribution (shown in Fig. 5) is now calculated through two loops. The single loop result

$$
\begin{aligned}
a_{\mu}^{\mathrm{EW}(1)} & =\frac{G_{F}}{\sqrt{2}} \frac{m_{\mu}^{2}}{8 \pi^{2}}\{\underbrace{\frac{10}{3}}_{W}+\underbrace{\frac{1}{3}\left(1-4 \sin ^{2} \theta_{W}\right)^{2}-\frac{5}{3}}_{Z} \\
& \left.+\mathcal{O}\left(\frac{m_{\mu}^{2}}{M_{Z}^{2}} \log \frac{M_{Z}^{2}}{m_{\mu}^{2}}\right)+\frac{m_{\mu}^{2}}{M_{H}^{2}} \int_{0}^{1} d x \frac{2 x^{2}(2-x)}{1-x+\frac{m_{\mu}^{2}}{M_{H}^{2}} x^{2}}\right\} \\
& =194.8 \times 10^{-11}
\end{aligned}
$$

was calculated by five separate groups shortly after the Glashow-Salam-Weinberg theory was shown by 't Hooft to be renormalizable. With the present limit on the Higgs boson mass, only the $W$ and $Z$ contribute at a measurable level. 


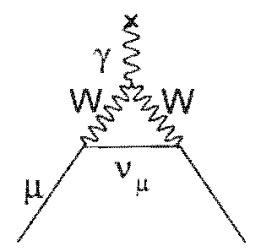

(a)

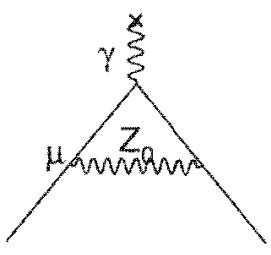

(b)

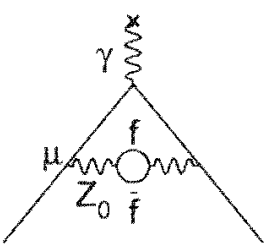

(c)

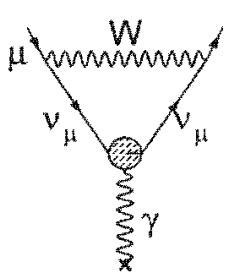

(d)

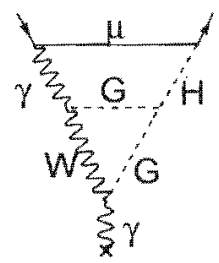

(e)

FIG. 5: Weak contributions to the muon anomalous magnetic moment. Single-loop contributions from (a) virtual $W$ and (b) virtual $Z$ gauge bosons. These two contributions enter with opposite sign, and there is a partial cancellation. The two-loop contributions fall into three categories: (c) fermionic loops which involve the coupling of the gauge bosons to quarks, (d) bosonic loops which appear as corrections to the one-loop diagrams, and (e) a new class of diagrams involving the Higgs where $G$ is the longitudinal component of the gauge bosons. See Ref. [19] for details. The $x$ indicates the virtual photon from the magnetic field.

The two-loop weak contribution, (see Figs. 5(c-e) for examples) is negative, and the total electroweak contribution is

$$
a_{\mu}^{\mathrm{EW}}=152(1)(2) \times 10^{-11}
$$

where the first error comes from hadronic effects in the second-order electroweak diagrams with quark triangle loops, and the latter comes from the uncertainty on the Higgs mass[3, 19]. The leading logs for the next-order term have been shown to be small. The weak contribution is about 1.3 ppm of the anomaly, so the experimental uncertainty on $a_{\mu}$ of \pm 0.54 ppm now probes the weak scale of the standard model.

\section{The Hadronic Contribution}

The hadronic contribution to $a_{\mu}$ is about $60 \mathrm{ppm}$ of the total value. The lowest-order diagram shown in Fig. 6(a) dominates this contribution and its error, but the hadronic light-by-light contribution Fig. 6(e) is also important.

The energy scale for the virtual hadrons is of order $m_{\mu} c^{2}$, well below the perturbative region of $\mathrm{QCD}$. Thus it must be calculated from the dispersion relation shown pictorially in 


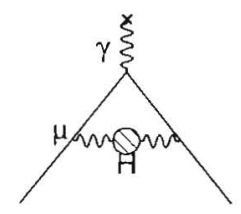

(a)

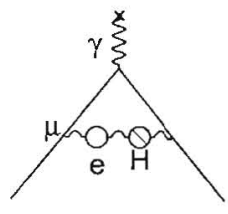

(b)

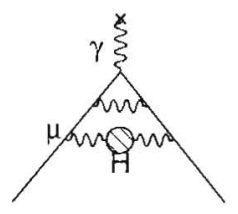

(c)

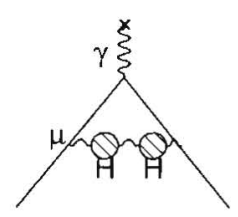

(d)

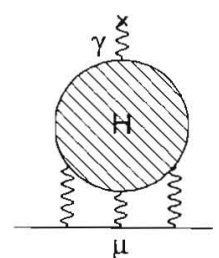

(e)

FIG. 6: The hadronic contribution to the muon anomaly, where the dominant contribution comes from the lowest-order diagram (a). The hadronic light-by-light contribution is shown in (e).

Fig. 7,

$$
a_{\mu}^{\text {had; LO }}=\left(\frac{\alpha m_{\mu}}{3 \pi}\right)^{2} \int_{4 m_{\pi}^{2}}^{\infty} \frac{d s}{s^{2}} K(s) R(s), \quad \text { where } \quad R \equiv \frac{\sigma_{\text {tot }}\left(e^{+} e^{-} \rightarrow \text { hadrons }\right)}{\sigma\left(e^{+} e^{-} \rightarrow \mu^{+} \mu^{-}\right)}
$$

using the measured cross sections for $e^{+} e^{-} \rightarrow$ hadrons as input, where $K(s)$ is a kinematic factor ranging from -0.63 at $s=4 m_{\pi}^{2}$ to 1 at $s=\infty$. This dispersion relation relates the bare cross section for $e^{+} e^{-}$annihilation into hadrons to the hadronic vacuum polarization contribution to $a_{\mu}$. Because the integrand contains a factor of $s^{-2}$, the values of $R(s)$

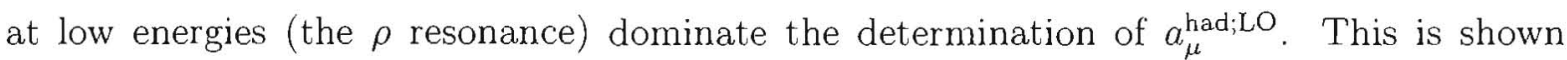
in Fig. 8, where the left-hand chart gives the relative contribution to the integral for the different energy regions, and the right-hand gives the contribution to the error squared on the integral. The contribution is dominated by the two-pion final state, but other low-energy multi-hadron cross sections are also important.

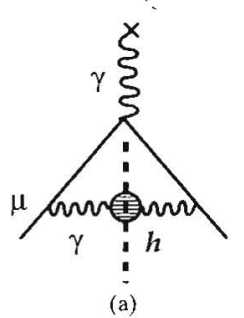

(a)

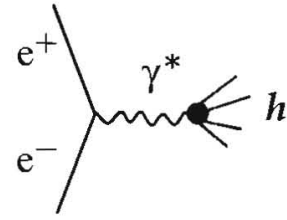

(b)

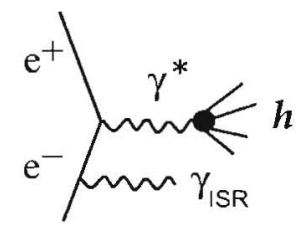

(c)

FIG. 7: (a) The "cut" hadronic vacuum polarization diagram; (b) The $e^{+} e^{-}$annihilation into hadrons; (c) Initial state radiation accompanied by the production of hadrons.

These data for $e^{+} e^{-}$annihilation to hadrons are also important as input into the determination of $\alpha_{s}\left(M_{Z}\right)$ and other electroweak precision measurements, including the limit on 


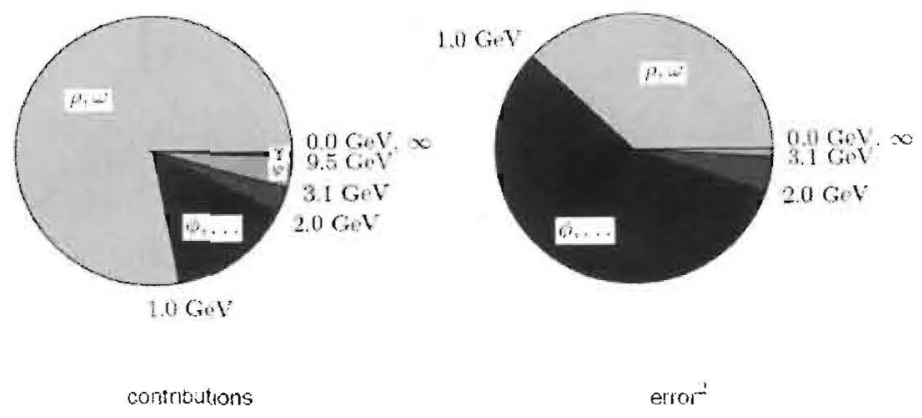

FIG. 8: Contributions to the dispersion integral, and to the error on the dispersion integral. Taken from F. Jegerlehner [20].

the Higgs mass[21]. After the discussion of the determination of the hadronic contribution, we will return to the implications on $M_{H}$.

In the 1980s when E821 was being proposed at Brookhaven, the hadronic contribution was know to about $10 \mathrm{ppm}$. It now is known to about $0.4 \mathrm{ppm}$. This improvement has come from the hard work of many experimental and theoretical physicists. The low energy $e^{+} e^{-}$ data of the 80s has been replaced by very precise data from the CMD2 and SND detectors in Novosibirsk, the KLOE collaboration at Frascati, and the BaBar collaboration at SLAC. Additional data are expected from the Belle detector at KEK. In addition to the collider experiments, significant theoretical work has been carried out in generating the radiator functions used in the initial-state radiation (ISR) experiments at Frascati and BaBar[22], as well as on the hadronic light-by-light contribution shown in Fig. 6(e). This work was carried out because E821 was being performed, and has continued well after E821 reported its final result. We emphasize that while this is a difficult subject, progress continues to be made, motivated by the present E821 result and by the possibility that a new experiment with even greater precision could be carried out at Fermilab.

\section{The Lowest-order Hadronic Contribution}

Because the cross sections at low energies dominate the dispersion relation, it is the lowenergy electron-positron storage rings in Novosibirsk, and Frascati that mostly contributed to the new measurements. At Novosibirsk, there are two experiments, the cryogenic magnetic 
detector (CMD2), and the spherical neutral detector (SND) which has no magnetic field. Both of these experiments have measured the hadronic cross section in a traditional energy scan. At Frascati, ISR (often called "radiative return") has been used, where the accelerator is operated at around $1 \mathrm{GeV}$ (mostly on the $\phi$ resonance), using hadronic events accompanied by an initial-state (ISR) photon (see Fig. 7(c)) to measure the hadronic cross-section. More recently the BaBar experiment has used ISR to measure the hadronic cross sections, and their data on multi-hadron final states have been published. The BaBar data, taken at the PEP2 collider, differ from the lower-energy experiments at KLOE, since the initial-state photon is quite energetic and easily detected. The Belle experiment at KEK is also beginning to look at ISR data, and we assume that they too will join this effort.

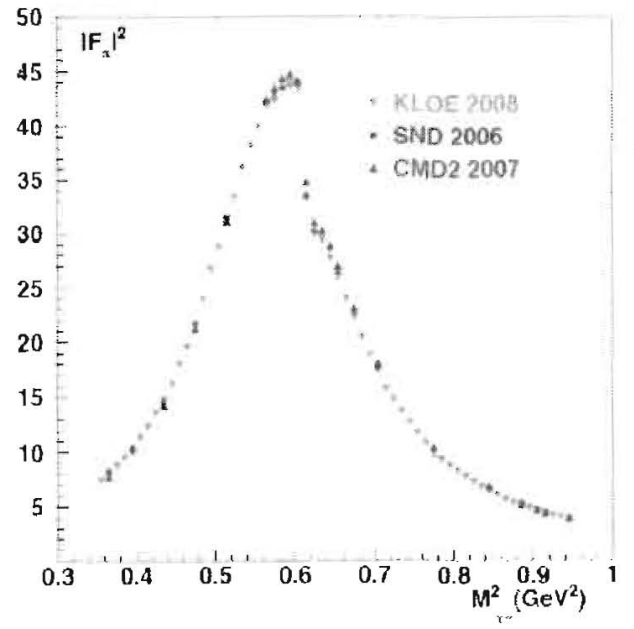

only statistical errors are shown

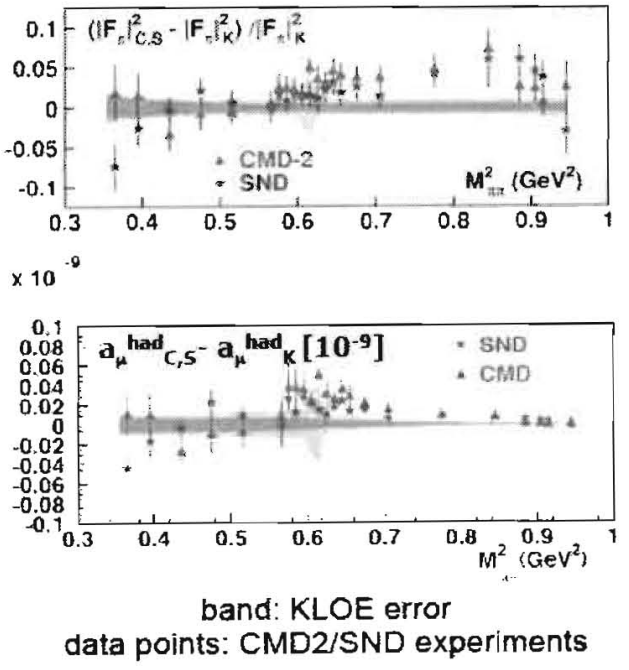

CMD-2 and SND data have been averaged over width of KLOE bin $\left(0.01 \mathrm{GeV}^{2}\right)$

FIG. 9: The pion form factor $\left|F_{\pi}\right|^{2}$ from KLOE, SND and CMD2. The upper right-hand side shows a comparison of the shape of the pion form factor from CMD2 and SND compared to that from KLOE. The bottom right shows the comparison of the (weighted) contribution to the dispersion integral. The largest differences correspond to about $1 \times 10^{-11}$ in the value of the dispersion integral.

As mentioned above, the two-pion final state is the most important contributor to the dispersion integral. Published results from the KLOE, CMD2 and SND experiments are 
shown in Fig. 9. It is traditional to report the pion form factor $F_{\pi}$, defined by

$$
\sigma_{e^{+} e^{-} \rightarrow \pi^{+} \pi^{-}}=\frac{\pi \alpha^{2}}{3 s} \beta_{\pi}^{3}\left|F_{\pi}\right|^{2}
$$

which is shown below for KLOE, CMD2 and SND.

A recent analysis [27] gives:

$$
a_{\mu}^{\text {hvp }}=\left(6908 \pm 39_{\exp } \pm 19_{\text {rad }} \pm 7_{\mathrm{QCD}}\right) \times 10^{-11} .
$$

which represents the effort of many experimental groups. Important earlier global analyses include those of HMNT [23], Davier [24], Jegerlehner[25] and Höcker and Marciano[26]. Following de Rafael, we use the recent analysis of Zhang [27], which however does not include the new results from the KLOE experiment [28] which were published after Zhang's review. The next-order hadronic contribution shown in Fig. 6(b-d) can also be determined from a dispersion relation, and the result is

$$
a_{\mu}^{\mathrm{hvp}(\text { nlo })}=\left(-97.9 \pm 0.9_{\exp } \pm 0.3_{\mathrm{rad}}\right) \times 10^{-11}
$$

\section{BaBar $e^{+} e^{-} \rightarrow \pi^{+} \pi^{-}$Data}

In September 2008, preliminary results from the $\pi \pi$ final state using the radiative return method were reported by the BaBar collaboration [29]. At the time of this proposal, they remain preliminary and cannot yet be included in the dispersion relation. Davier, in his 30 January, 2009 seminar at Fermilab, announced that a systematic problem was discovered in the threshold region, which affects the previously announced cross section measurements in the higher-mass region. The $\mathrm{BaBar}$ Collaboration is completing their work now and will make final results available in the next few months. Davier pointed out that the uncertainty on the lowest-order hadronic contribution from "existing $e^{+} e^{-}$data (all experiments) can reach a precision $\delta_{a_{\mu}}=25 \times 10^{-11 "}$ and he expressed optimism that "remaining discrepancies will be brought close to quoted systematic uncertainties."

5. $a_{\mu}^{\text {had; } \mathrm{LO}}$ from Hadronic $\tau$ decay

The value of $a_{\mu}^{\text {had; LO }}$ from threshold up to $m_{\tau}$ could in principle be obtained from hadronic $\tau^{-}$decays (See Fig. 6), provided that the necessary isospin corrections are known. This 
was first demonstrated by Almany, Davier and Höcker [30]. Hadronic $\tau$ decays to an even number of pions such as $\tau^{-} \rightarrow \pi^{-} \pi^{0} \nu_{\tau}$, which in the absence of second-class currents goes through the vector part of the weak current, can be related to $e^{+} c$ annihilation into $\pi^{+} \pi^{-}$ through the CVC hypothesis and isospin conservation (see Fig. 10) [30-32]. The $\tau$-data only contain an isovector piece, and the isoscalar piece present in $e^{-t} e^{-}$annihilation has to be put in "by hand" to evaluate $a_{\mu}^{\text {had:LO }}$. At present, most authors $[3,20,23]$ conclude that there are unresolved issues such as the isospin breaking corrections, which make it difficult to use the $\tau$ data on an equal footing with the $e^{+} e^{-}$data.

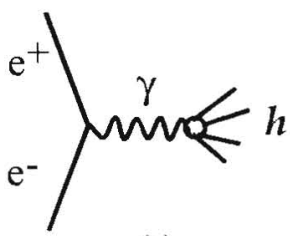

(a)

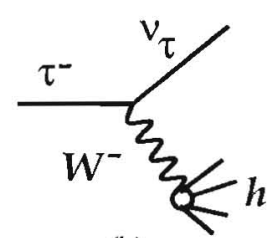

(b)

FIG. 10: $e^{+} e^{-}$annihilation into hadrons (a), and hadronic $\tau$ decay (b).

\section{The Hadronic Light-by-Light Contribution}

The hadronic light-by-light contribution, (Fig. 6(e)) cannot at present be determined from data, but rather must be calculated using hadronic models that correctly reproduce the properties of QCD. A number of authors have calculated portions of this contribution, and recently a compilation of all contributions has become available from Prades, de Rafael and Vainshtein [33], which has been agreed to by authors from each of the leading groups working in this field. They obtain

$$
a_{\mu}^{\text {HLbL }}=(105 \pm 26) \times 10^{-11}
$$

Additional work on this contribution is underway at Minnesota [34].

\section{B. Summary of the Standard Model Value and Comparison with Experiment}

Following de Rafael [3], the standard-model value obtained from the published $e^{+} e^{-}$data from KLOE, CMD2 and SND, and published data from BaBar for the multi-pion final states is used to determine $a_{\mu}^{\text {hvp }}$ and $a_{\mu}^{\text {hvp(nlo) }}$. A summary of these values is given in Table III. 
TABLE III: Standard model contributions to the muon anomaly. Taken from de Rafael[3].

\begin{tabular}{lr}
\hline Contribution & Result IN $10^{-11}$ Units \\
\hline QED (leptons) & $116584718.09 \pm 0.14 \pm 0.04_{\alpha}$ \\
HVP(lo) & $6908 \pm 39_{\text {exp }} \pm 19_{\text {rad }} \pm 7_{\mathrm{pQCD}}$ \\
HVP(ho) & $-97.9 \pm 0.9_{\text {exp }} \pm 0.3_{\mathrm{rad}}$ \\
$\mathrm{HLxL}$ & $105 \pm 26$ \\
$\mathrm{EW}$ & $152 \pm 2 \pm 1$ \\
\hline Total SM & $116591785 \pm 51$ \\
\hline
\end{tabular}

This standard-model value is to be compared with the combined $a_{\mu}^{+}$and $a_{\mu}^{-}$values from E821 [2]:

$$
\begin{aligned}
a_{\mu}^{\mathrm{E} 821} & =(116592080 \pm 63) \times 10^{-11} \quad(0.54 \mathrm{ppm}) \\
a_{\mu}^{\mathrm{SM}} & =(116591785 \pm 51) \times 10^{-11}(0.44 \mathrm{ppm})
\end{aligned}
$$

which give a difference of

$$
\Delta a_{\mu}(\mathrm{E} 821-\mathrm{SM})=(295 \pm 81) \times 10^{-11}
$$

This comparison is shown graphically in Fig. 11.

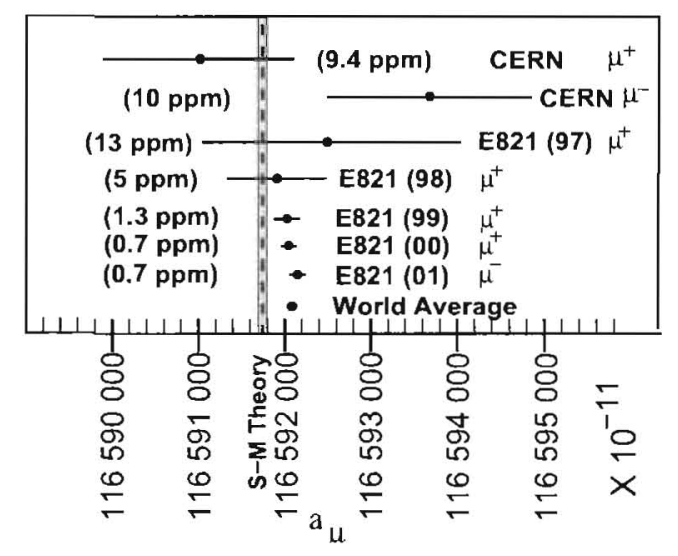

FIG. 11: Measurements of $a_{\mu}$ along with the standard-model value given above. 
This difference of 3.6 standard deviations is tantalizing, but we emphasize that whatever the final agreement between the measured and standard-model value turns out to be, it will have significant implications on the interpretation of new phenomena that might be found at the LHC and elsewhere. This point is discussed in detail below.

The present theoretical error [3] of $\pm 51 \times 10^{-11}(0.44 \mathrm{ppm})$ is dominated by the $\pm 39 \times 10^{-11}$ uncertainty on the lowest-order hadronic contribution and the $\pm 26 \times 10^{-11}$ uncertainty on the hadronic light-by-light contribution. As mentioned above, Davier suggested that the uncertainty on the lowest-order hadronic contribution could be reduced to $25 \times 10^{-11}$ with "potentially existing data". Future work described below could lower this further, and along with future theoretical progress on the hadronic light-by-light contribution, the total standard-model error could reach $30 \times 10^{-11}$.

With the proposed experimental error of $\pm 16 \times 10^{-11}$, the combined uncertainty for the difference between theory and experiment would be $\pm 34 \times 10^{-11}$, which is to be compared with the $\pm 81 \times 10^{-11}$ in Eq. 28 .

\section{1. $R(s)$ Measurements and the Higgs Mass, $M_{H}$}

If the hadronic cross section that enters into the dispersion relation of $\mathrm{Eq}$. 21 were to increase significantly from the value obtained in the published papers of CMD2, SND and KLOE, then as pointed out by Passera, Marciano and Sirlin [21], it would have significant implications for the limit on the mass of the Higgs boson. The value of $\Delta \alpha_{\text {had }}^{(5)}\left(M_{Z}\right)$ depends on the same measured cross-sections that enter into Eq. 21,

$$
\Delta \alpha_{\text {had }}^{(5)}\left(M_{Z}\right)=\frac{M_{Z}^{2}}{4 \alpha \pi^{2}} P \int_{4 m_{\pi}^{2}}^{\infty} d s \frac{\sigma(s)}{M_{Z}^{2}-s} .
$$

The present bound of $M_{H} \leq 150 \mathrm{GeV}\left(95 \%\right.$ C.L.) changes if $\Delta \alpha_{\text {had }}\left(M_{Z}\right)$ changes. Assuming that the hadronic contribution to $a_{\mu}$ is increased by the amount necessary to remove the difference between the experimental and theoretical values of $a_{\mu}$, the effect on $M_{H}$ is to move the upper bound down to $\simeq 130 \mathrm{GeV}$. Given the experimental limit $M_{H}>114.4 \mathrm{GeV}$ $(95 \%$ C.L.), this significantly narrows the window for the Higgs mass: The details depend on the s-region assumed to be incorrect in the hadronic cross section. A much more complete discussion is given in Ref. [21]. 


\section{Expected Improvements in the Standard Model Value}

Much experimental and theoretical work is going on worldwide to refine the hadronic contribution. One reflection of this effort is the workshop held in Glasgow [35], which brought together 27 participants who are actively working on parts of this problem, including the beyond the standard model implications of $a_{\mu}$. These participants represented many additional collaborators.

In the near term there will be two additional results from KLOE, in addition to the final $\mathrm{BaBar}$ result. An upgrade at Novosibirsk is now underway.

- Novosibirsk: The CMD2 collaboration has upgraded their detector to CMD3, and the VEPP2M machine has been upgraded to VEPP-2000. The maximum energy has been increased from $\sqrt{s}=1.4 \mathrm{GeV}$ to $2.0 \mathrm{GeV}$. These upgrades will permit the cross section to be measured from threshold to $2.0 \mathrm{GeV}$ using an energy scan, filling in the energy region between $1.4 \mathrm{GeV}$, where the CMD2 scan ended, up to $2.0 \mathrm{GeV}$, the lowest energy point reached by the BES collaboration in their measurements. See Fig. 8 for the present contribution to the overall error from this region. The SND detector has also been upgraded. Engineering runs will take place in 2009, with data collection beginning in late 2009 or 2010 . They will also take data at $2 \mathrm{GeV}$, using ISR, which will provide data between the PEP2 energy at the $\Upsilon(4 s)$ and the $1 \mathrm{GeV} \phi$ energy at the DA $\phi N E$ facility in Frascati.

- KLOE: The KLOE collaboration has measured the hadronic cross section using initial-state radiation (ISR) to lower the CM energy from the $\phi$ where DA $\phi \mathrm{NE}$ operates. Significant data have now been published. Several additional analyses are in progress: a measurement of the pion form factor from the bin by bin ratio of $\pi^{+} \pi^{-} \gamma$ to $\mu^{+} \mu^{-} \gamma$ spectrum, (as is being done with the BaBar analysis) large-angle ISR data; and ISR data taken off of the $\phi$ resonance, with a different data set, analysis selection, and background conditions.

- BaBar: As discussed above, the BaBar collaboration is several months from reporting the final analysis of their $\pi^{+} \pi^{-}$ISR data. They have significantly more data, but at present, no member of the collaboration has taken on the leadership of that analysis effort. 
- Belle: Some work on ISR measurements of $R(s)$ is going on but at present they are not nearly as far along as BaBar.

- Calculations on the Lattice - Lowest-Order: With the increased computer power available for lattice calculations, it may be possible for lattice calculations to contribute to our knowledge of the lowest order hadronic contribution. Blum has performed a proof-of-principle quenched calculation on the lattice.[36, 37] Several groups, UKQCD (Edinburg), DESY-Zeuthen (Renner and Jansen), and the LSD (lattice strong dynamics) group in the US are all working on the lowest-order contribution.

- Calculations on the Lattice - Hadronic light-by-light: The hadronic lightby-light contribution has a magnitude of $(105 \pm 26) \times 10^{-11}, \sim 1$ ppm of $a_{\mu}$. A modest calculation on the lattice would have a large impact. There are two separate efforts to formulate the hadronic light-by-light calculation on the lattice. Blum and his collaborators at BNL and RIKEN (RBC collaboration) are working on the theoretical framework for a lattice calculation of this contribution, and are calculating the QED light-by-light contribution as a test of the program.[38]

\section{Physics Beyond the Standard Model}

For many years, the muon anomaly has played an important role in constraining physics beyond the standard model [39 42]. The 1260 citations to the major E821 papers [2, 10-12], with 164 in 2008 , demonstrates that this role continues.

In this section, we discuss how the muon anomaly provides a unique window to search for physics beyond the standard model. If new physics is discovered at the LHC, then $a_{\mu}$ will play an important role in sorting out the interpretation of those discoveries. In the sections below, examples of constraints placed on various models that have been proposed as extensions of the standard model are discussed. However, perhaps the ultimate value of an improved limit on $a_{\mu}$, will come from its ability to constrain the models that we have yet invented.

If the LHC produces the standard model Higgs and nothing else, then the only tools available to probe the high-energy frontier will be precision measurements of $a_{\mu}$ or other weak processes, together with searches for charged lepton flavor violation, electric dipole 
moments, and rare decays.

The role of $(g-2)$ as a discriminator between very different standard model extensions is well illustrated by a relation discussed by Czarnecki and Marciano [40] that holds in a wide range of models: If a new physics model with a mass scale $\Lambda$ contributes to the muon mass $\delta m_{\mu}$ (N.P.), it also contributes to $a_{\mu}$, and the two contributions are related as

$$
a_{\mu}(\text { N.P. })=\mathcal{O}(1) \times\left(\frac{m_{\mu}}{\Lambda}\right)^{2} \times\left(\frac{\delta m_{\mu}(\mathrm{N} . \mathrm{P} .)}{m_{\mu}}\right)
$$

The ratio $C$ (N.P.) $\equiv \delta m_{\mu}$ (N.P.) $/ m_{\mu}$ is typically between $\mathcal{O}(\alpha / 4 \pi)$ (for perturbative contributions to the muon mass) and $\mathcal{O}(1)$ (if the muon mass is essentially due to radiative corrections). Hence the contributions to $a_{\mu}$ are highly model dependent.

A variety of models with radiative muon mass generation at some scale $\Lambda$ have been discussed in [40], including extended technicolor or generic models with naturally vanishing bare muon mass. In these models the ratio $C$ (N.P.) $\simeq 1$ and the new physics contribution to $a_{\mu}$ can be very large,

$$
a_{\mu}(\Lambda) \simeq \frac{m_{\mu}^{2}}{\Lambda^{2}} \simeq 1100 \times 10^{-11}\left(\frac{1 \mathrm{TeV}}{\Lambda}\right)^{2}
$$

and the difference Eq. 3 can be used to place a lower limit on the new physics mass scale, which is in the few $\mathrm{TeV}$ range [43]. In models with extra weakly interacting gauge bosons $Z^{\prime}, W^{\prime}$, e.g. certain models with extra dimensions, $C($ N.P. $)=\mathcal{O}(\alpha / 4 \pi)$, and a difference as large as Eq. 3 is very hard to accommodate unless the mass scale is very small, of the order of $M_{Z}$. In a model with $\delta=1$ (or 2) universal extra dimensions, other measurements already imply a lower bound of 300 (or 500) GeV on the masses of the extra states, and the one-loop contributions to $a_{\mu}$ are correspondingly small,

$$
a_{\mu}(\mathrm{UED}) \simeq-5.8 \times 10^{-11}(1+1.2 \delta) S_{\mathrm{KK}}
$$

with $\left|S_{K K}\right|_{\sim}^{<1}$ [44]. Many other models with extra weakly interacting particles give similar results [45]. If any of these are realized in Nature, the new measurement of $a_{\mu}$ would be expected to agree with the standard model value within approximately $\pm 34 \times 10^{-11}$, the projected sensitivity of the combined standard model plus experiment sensitivity.

Supersymmetric models lie in between these two extremes. Were they to exist, muon $(g-2)$ would have substantial sensitivity to the supersymmetric particles. Compared to generic perturbative models, supersymmetry provides an enhancement to $C$ (SUSY) $=$ 
$\mathcal{O}(\tan \beta \alpha / 4 \pi)$ and to $a_{\mu}(\mathrm{SUSY})$ by a factor $\tan \beta$ (the ratio of the vacuum expectation values of the two Higgs fields). The SUSY diagrams for the magnetic dipole moment, the electric dipole moment, and the lepton-number violating conversion process $\mu \rightarrow e$ in the field of a nucleus are shown pictorially in Fig. 12. In a model with SUSY masses equal to $\Lambda$ the supersymmetric contribution to $a_{\mu}$ is given by [40]

$$
a_{\mu}(\mathrm{SUSY}) \simeq \operatorname{sgn}(\mu) 130 \times 10^{-11} \tan \beta\left(\frac{100 \mathrm{GeV}}{\Lambda}\right)^{2}
$$

which indicates the dependence on $\tan \beta$, and the SUSY mass scale, as well as the sign of the SUSY $\mu$-parameter. Thus muon $(g-2)$ is sensitive to any SUSY model with large $\tan \beta$. Conversely, SUSY models with $\Lambda$ in the few hundred GeV range could provide an explanation of the deviation in Eq. 3.

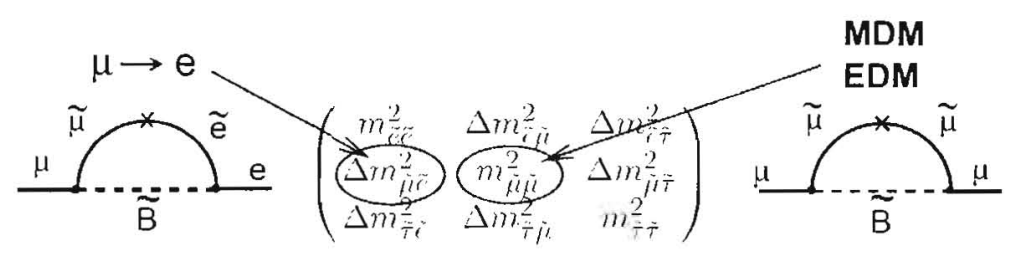

FIG. 12: The supersymmetric contributions to the anomaly, and to $\mu \rightarrow e$ conversion, showing the relevant slepton mixing matrix elements. The MDM and EDM give the real and imaginary parts of the matrix element, respectively. The $x$ indicates a chirality flip.

In the next decade, LHC experiments will for the first time directly probe physics at the $\mathrm{TeV}$ scale. This scale appears to be a crucial scale in particle physics. It is linked to electroweak symmetry breaking, and many arguments indicate that radically new concepts such as supersymmetry, extra dimensions, technicolor, or other new interactions, could be realized at this scale. Furthermore, cold dark matter particles could have weak-scale/TeV-scale masses, and if it exists, models of Grand Unification prefer the existence of supersymmetry at the TeV scale. TeV-scale physics could be very rich, and the LHC is designed to discover physics beyond the standard model. This will make the precision experiments such as muon $(g-2)$ and searches for charged lepton flavor violation complementary partners

In the quest to identify the nature of $\mathrm{TeV}$-scale physics and to answer questions related to e.g. electroweak symmetry breaking and Grand Unification, we need to combine and crosscheck information from the LHC with information from as many complementary experiments 
as possible. This need is highlighted by the unprecedented complexity of the LHC accelerator and experiments, the involved initial and final states, and the huge backgrounds at the LHC. In all these respects, an improved muon $(g-2)$ measurement is needed to provide an indispensable complement.

In the following we discuss in more detail how $a_{\mu}$ will be useful in understanding TeVscale physics in the event that the LHC established the existence of physics beyond the standard model [46].

\section{1. $a_{\mu}$ as a benchmark for models of new physics}

It has been established that the LHC is sensitive to virtually all proposed weak-scale extensions of the standard model, ranging from supersymmetry (SUSY) to extra dimensions, little Higgs models and others. However, even if the existence of physics beyond the standard model is established, it will be far from easy for the LHC alone to identify which of the possible alternatives is realized. The measurement of $a_{\mu}$ to $16 \times 10^{-11}$ will be highly valuable in this respect since it will provide a benchmark and stringent selection criterion that can be imposed on any model that is tested at the LHC.

For example, a situation is possible where the LHC finds many new heavy particles which are compatible with both minimal-supersymmetric and universal-extra-dimension model predictions [47]. The muon $(g-2)$ would especially aid in the selection since UED models predict a tiny effect to $a_{\mu}$, while SUSY effects are usually much larger.

Likewise, within SUSY itself there are many different well-motivated scenarios that are not always easy to distinguish at the LHC. Fig. 13 shows a graphical distribution of the 10 Snowmass Points and Slopes model benchmark predictions [48] for $a_{\mu}$ (SUSY). They range considerably and can be positive and negative, due to the factor $\operatorname{sgn}(\mu)$ in $\mathrm{Eq} .33$, where this sign would be particularly difficult to determine at LHC, even if SUSY were to be discovered. The discriminating power of an improved $(g-2)$ measurement even if the actual value of $\Delta a_{\mu}$ turned out to be smaller-is evident from Fig. 13.

A final example concerns the restriction of special, highly constrained models of new physics such as the constrained MSSM (CMSSM). The CMSSM has only four free continuous parameters. One precise measurement such as the future determination of $\Delta a_{\mu}$ effectively fixes one parameter as a function of the others and thus reduces the number of 


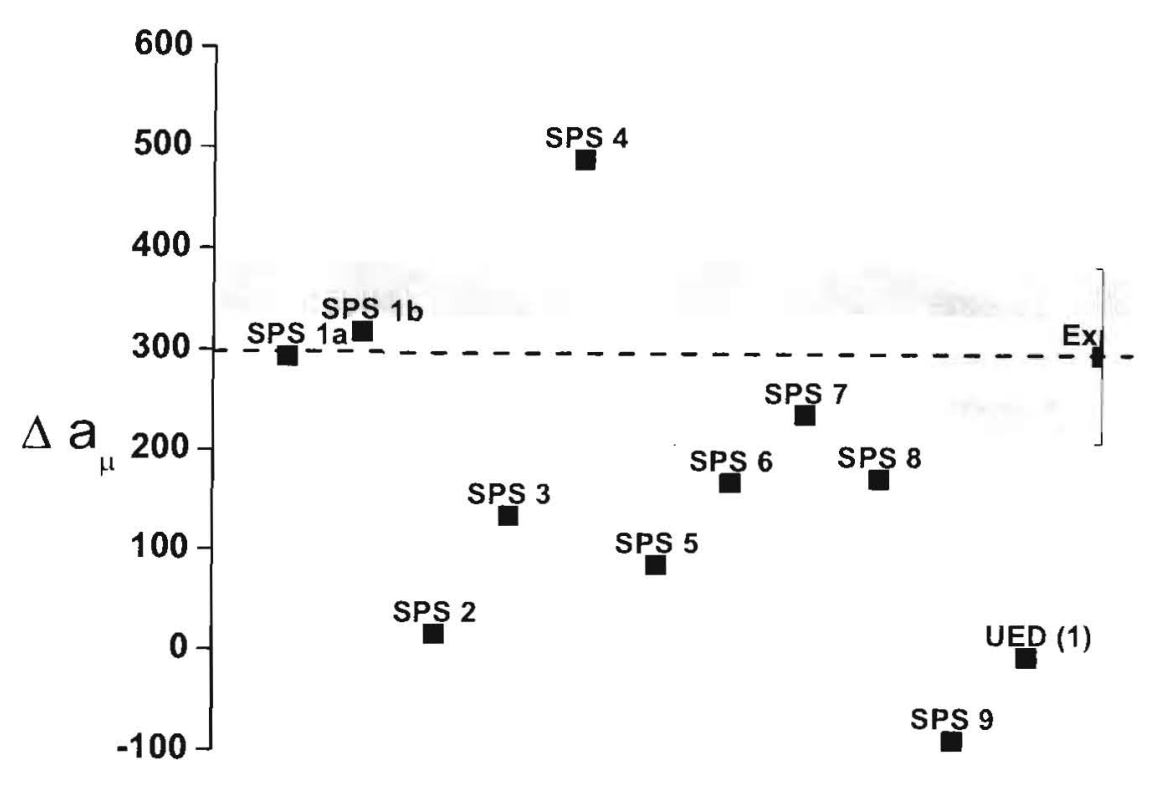

(a)

FIG. 13: The Snowmass Points and Slopes predictions for $a_{\mu}$ (SUSY) (in units of $10^{-11}$ ) for various scenarios [48], and the UED prediction for one extra dimension [44]. (The horizontal axis has no meaning except to make all points visible.) The wide blue band is the present $1 \sigma$ difference between experiment and theory. The narrow yellow band represents the proposed improved precision, given the same central value.

free parameters by one. A large number of recent analyses have made use of this feature, see e.g. Refs. [49]. In fact, the CMSSM is very sensitive not only to the $a_{\mu}$ but also to the dark matter (which in this model is assumed to consist of neutralinos) relic density. As shown in Fig. 14, both observables lead to orthogonal constraints in CMSSM parameter space, and therefore imposing both constraints leaves only two free parameters and thus allows for very stringent tests of the CMSSM at the LHC. From Fig. 14(a) we see that in this model, there is little room left for $\tan \beta=10$. 


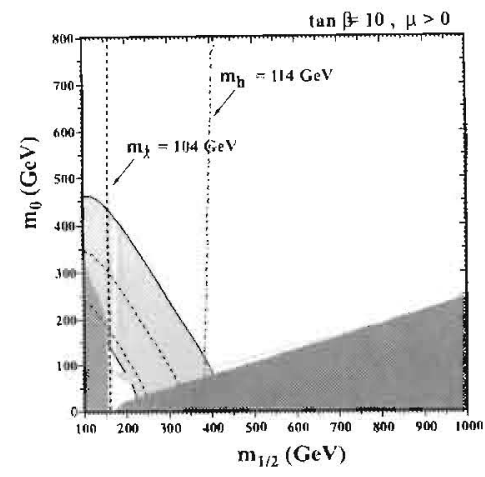

(a)

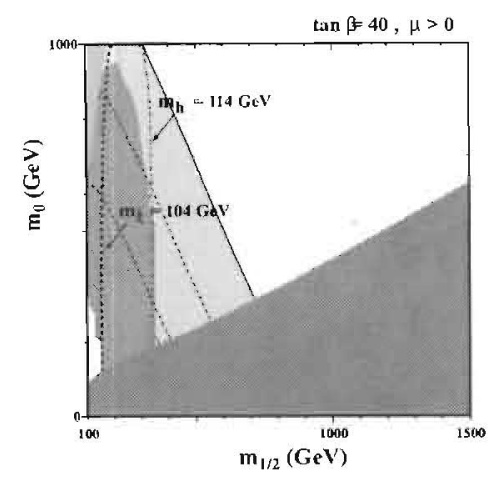

(d)

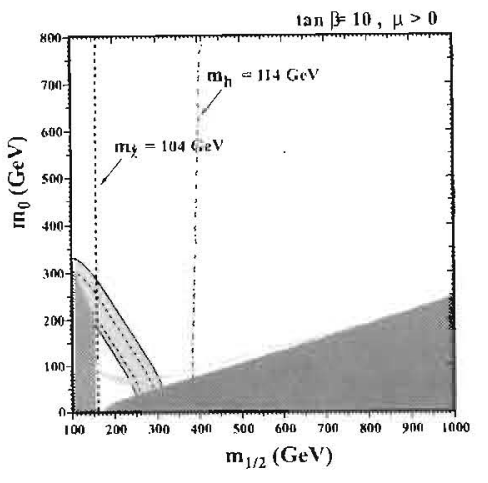

(b)

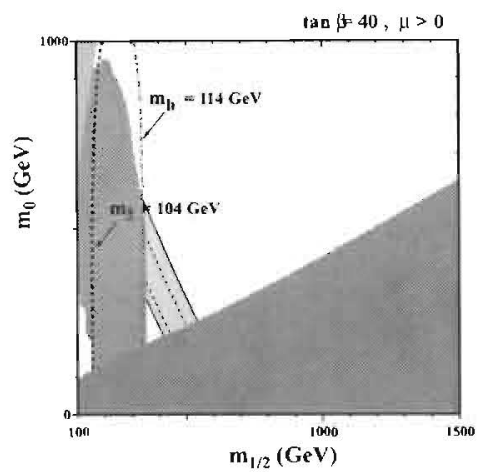

(e)

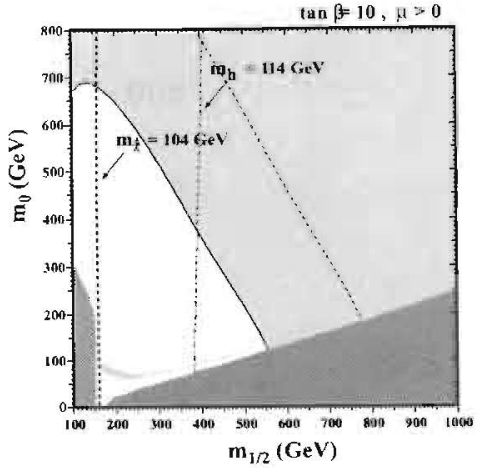

(c)

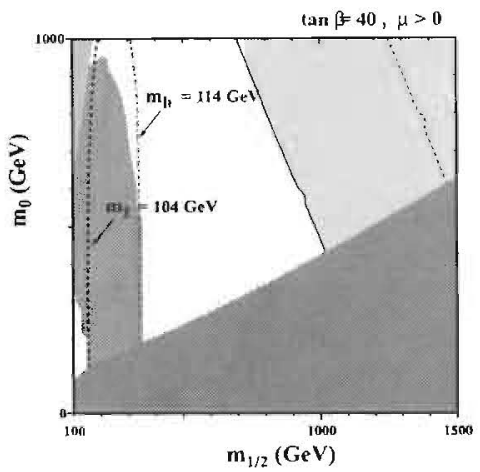

(f)

FIG. 14: The $m_{0}$ (scalar mass) $-m_{1 / 2}$ (gaugino mass) plane of the CMSSM parameter space for $\tan \beta=(10 ; 40), A_{0}=0, \operatorname{sgn}(\mu)=+$ :

(a;d) The $\Delta a_{\mu}^{\text {(today) }}=295(81) \times 10^{-11}$ between experiment and standard-model theory is from Ref. [3]. The brown wedge on the lower right is excluded by the requirement the dark matter be neutral. Direct limits on the Higgs and chargino $\chi^{ \pm}$masses are indicated by vertical lines, with the region to the left excluded. Restrictions from the WMAP satellite data are shown as a light-blue line. The $(g-2) 1$ and 2-standard deviation boundaries are shown in purple. The green region is excluded by $b \rightarrow s \gamma$. (b;e) The plot with $\Delta a_{\mu}=295(39) \times 10^{-11}$. (c;f) The same errors as (b), but $\Delta a_{\mu}=0$. (Figures courtesy of K. Olive, following Ref. [50])

\section{2. $a_{\mu}$ is sensitive to quantities that are difficult to measure at the $L H C$}

As a hadron collider, the LHC is particularly sensitive to colored particles. In contrast, $a_{\mu}$ is particularly sensitive to weakly interacting particles that couple to the muon and to 
new physics effects on the muon mass, see Eq. 30.

For unraveling the mysteries of $\mathrm{TeV}$-scale physics it is not sufficient to determine which type of new physics is realized, but it is necessary to determine model parameters as precisely as possible. Here the complementarity between the LHC and precision experiments such as $a_{\mu}$ becomes particularly important. A difficulty at the LHC is the very indirect relation between LHC observables (cross sections, mass spectra, edges, etc) and model parameters such as masses and couplings, let alone more underlying parameters such as supersymmetry-breaking parameters or the $\mu$-parameter in the MSSM. Generally, the LHC Inverse problem [51] states that several different points in the supersymmetry parameter space can give rise to indistinguishable LHC signatures. It has been shown that a promising strategy is to determine the model parameters by performing a global fit of a model such as the MSSM to all available LHC data. However, recent investigations have revealed that in this way typically a multitude of almost degenerate local minima of $\chi^{2}$ as a function of the model parameters results [52]. Independent observables such as the ones available at the proposed International Linear Collider [53] or $a_{\mu}$ will be highly valuable to break such degeneracies, and in this way to unambiguously determine the model parameters.

In the following we provide further examples for the complementarity of LHC and $a_{\mu}$ for the well-studied case of the MSSM. The LHC has only a weak sensitivity to two central parameters: the sign of the $\mu$-parameter and $\tan \beta$, the ratio of the two Higgs vacuum expectation values. According to Eq. 33 the MSSM contributions to $a_{\mu}$ are highly sensitive to both of these parameters. Therefore, a future improved $a_{\mu}$ measurement has the potential to establish a definite positive or negative sign of the $\mu$-parameter in the MSSM, which would be a crucial piece of information.

In the event that SUSY is discovered, we give an illustration of a $\tan \beta$ measurement and reconsider the case discussed in Ref. [52], assuming that the MSSM reference point SPS1a [48] is realized at LHC. Using the comprehensive LHC-analysis of [52], $\tan \beta$ can be determined only rather poorly to $\tan \beta^{\mathrm{LHC}}$ fit $=10.0 \pm 4.5$. In such a situation one can study the MSSM prediction for $a_{\mu}$ as a function of $\tan \beta$ (all other parameters are known from the global fit to LHC data) and compare it to the measured value, in particular after an improved measurement. As can be seen from Fig. 15, using today's value for $a_{\mu}$ would improve the determination of $\tan \beta$, but the improvement will be even more impressive after a future more precise $a_{\mu}$ measurement. The limits on $\tan \beta$ are: $\mathrm{E} 821 \tan \beta=9.8_{-3.4}^{+3.9}$; FNAL 
$\tan \beta=9.8_{-2.0}^{+2.1}$. Should such a scenario unfold, as the SUSY masses become better measured, the measure of $\tan \beta$ from $a_{\mu}$ would improve further. A similar but more comprehensive study in [54], where $a_{\mu}$ has been incorporated into the global fit, confirms this role of $a_{\mu}$ as an excellent observable to measure $\tan \beta$.

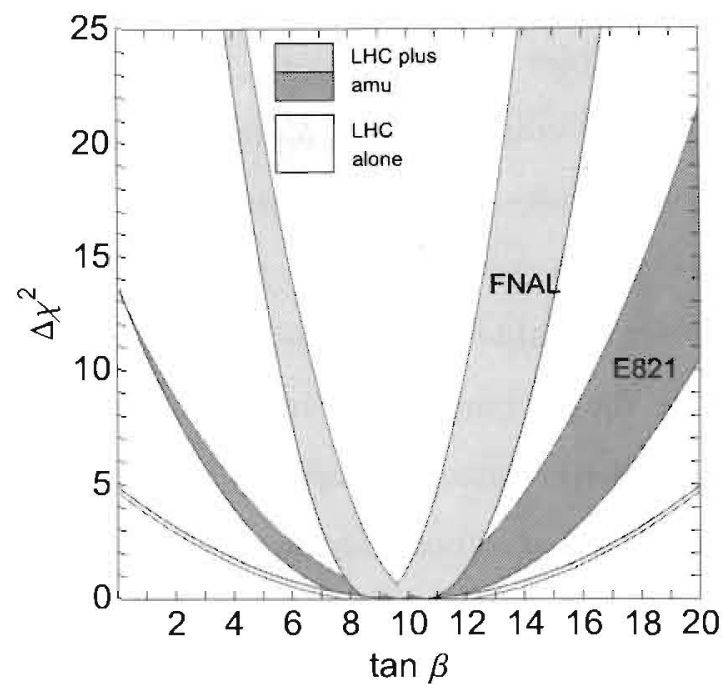

FIG. 15: Possible future $\tan \beta$ determination from the measurement of $a_{\mu}$, assuming that the MSSM reference point SPS1a is realized. The yellow band is from LHC alone which gives $\tan \beta^{\mathrm{LHC}}$ fit $=$ $10.0 \pm 4.5[52,54]$. The darker blue band labelled E821 assumes $\Delta a_{\mu}=(295 \pm 81) \times 10^{-11}$, which comes from the present values for $a_{\mu}$ and the Standard-Model contribution, the lighter blue band labelled FNAL corresponds to $\Delta a_{\mu}^{\text {futurc }}=295(34) \times 10^{-11}$. The blue bands show $\Delta \chi^{2}=$ $\left(\frac{a_{\mu}^{M S S M}(\tan \beta)-a_{\mu}^{\exp }}{\{81 ; 34\} \times 10^{-11}}\right)^{2}$ as a function of $\tan \beta$, where in $a_{\mu}^{\mathrm{MSSM}}(\tan \beta)$ all parameters except $\tan \beta$ have been set to the values determined at the LHC. The width of the blue curves results from the expected LHC-uncertainty of the parameters (mainly smuon masses and $M_{2}, \mu$ ) [54]. The plot shows that the precision for $\tan \beta$ that can be obtained using $a_{\mu}$ is limited by the precision of the other input parameters but is still much better than the determination using LHC data alone.

One should note that even if better ways to determine $\tan \beta$ at the LHC alone might be found, an independent determination using $a_{\mu}$ will still be highly valuable, as $\tan \beta$ is one of the central MSSM parameters; it appears in all sectors and in almost all observables. Therefore, measuring $\tan \beta$ in two different ways, e.g. using certain Higgs- or $b$-decays at the LHC and using $a_{\mu}$, would constitute a non-trivial and indispensable test of the universality 
of $\tan \beta$ and thus of the structure of the MSSM.

At the 2007 Glasgow $(g-2)$ Workshop [35], Martin and Wells presented an update of their so-called "superconservative analysis" [56], where a very conservative $5 \sigma$ band around the observed difference Eq. 3 and the general supersymmetric standard model are considered. Surprisingly, it could be shown that even this mild assumption leads to regions of parameter space which are excluded by $(g-2)$ and nothing else. Hence, $(g-2)$ provides complementary information to collider, dark matter, or other low-energy observables. An improved $(g-2)$ measurement will be very useful-independent of the actual numerical result.

In a similar spirit, Berger, Gainer, Hewett and Rizzo [57] discussed "supersymmetry without prejudice." First a large set of supersymmetry parameter points ("models") in a 19-dimensional parameter space was identified, which was in agreement with many important existing experimental and theoretical constraints. Then the implications for observables such as $(g-2)$ were studied. The result for $(g-2)$ was rather similar to Fig. 13, although the context was far more general: the entire range $a_{\mu}^{\text {SUSY }} \sim(-100 \ldots+300) \times 10^{-11}$ was populated by a reasonable number of "models." Therefore, a precise measurement of $(g-2)$ to $\pm 16 \times 10^{-11}$ will be a crucial way to rule out a large fraction of models and thus determine supersymmetry parameters.

The anomalous magnetic moment of the muon is sensitive to contributions from a wide range of physics beyond the standard model. It will continue to place stringent restrictions on all of the models, both present and yet to be written down. Assuming that we will be so fortunate as to discover new phenomena in the LHC era, $a_{\mu}$ will constitute an indispensable tool to discriminate between very different types of new physics, especially since it is highly sensitive to parameters which are difficult to measure at the LHC. If we are unfortunate, then it represents one of the few ways to probe beyond the standard model. In either case, it will play an essential and complementary role in the quest to understand physics beyond the standard model at the TeV scale. This prospect is what motivates our collaboration to push forward with a new measurement. 


\section{A NEW $(g-2)$ EXPERIMENT}

\section{A. Scientific Goal}

The E821 results were based on three $\sim 2$-month-long running periods in 1999, 2000, and 2001. A total of $8.55 \times 10^{9}$ events were included in the final fitted samples. Combined, this leads to a relative statistical uncertainty of $0.46 \mathrm{ppm}$. The systematic uncertainties were, in general, reduced in each running year, and a final combined averages for the magnetic field measurement $(0.17 \mathrm{ppm})$ and spin-precession analysis $(0.21 \mathrm{ppm})$ were combined in quadrature with the statistical error to obtain the final overall relative uncertainty of $0.54 \mathrm{ppm}$. In absolute units, the experimental uncertainty on $a_{\mu}$ is $63 \times 10^{-11}$.

The goal of the New $(g-2)$ Experiment is a precision improvement on $a_{\mu}$ by a factor of 4 to $\delta a_{\mu}=16 \times 10^{-11},(0.14 \mathrm{ppm})$. This is an arrived at by assuming roughly equal statistical and systematic uncertainty goals. At $\delta a_{\mu}=16 \times 10^{-11}$, the uncertainty will be well below the theoretical error for the seeable future. In consultation with theorists who evaluate the SM contributions, we estimate that improved and larger HVP input data sets will reduce the uncertainty from 51 to 30 in $10^{-11}$ units. Our experimental precision will be well below theory, barring unforseen breakthroughs. The uncertainty on the comparison between experiment and theory, $\Delta a_{\mu k}$, will be reduced to $34 \times 10^{-11}$. This low-energy precision SM test will provide a powerful discriminator of new physics models.

An improvement by a factor of 4 is both scientifically compelling and technically achievable. To do so in less than 2 years of running, will require use of $6 / 20$ of the Booster batches, each subdivided fourfold leading to $18 \mathrm{~Hz}$ of storage ring fills (4 times the fill frequency at BNL). The use of a long decay beamline, with true-forward decay kinematics and an open inflector magnet, will serve to improve the muon storage efficiency per proton by a factor of more than 6 . A significant reduction in background will result from the long beamline. The design of the experiment aims at a systematic error reduction by an overall factor of 3 . The plan described below will achieve these stated goals.

\section{B. Key Elements to a New Experiment}

The New $(g-2)$ Experiment relies on the following improvements compared to the BNL E821 Experiment: 
1. Increasing the stored muon flux per incident proton,

2. Increasing the fill frequency (lowers the instantaneous rate),

3. Decreasing the hadron-induced flash at injection,

4. Improving the stored muon beam dynamics with a better kick into the ring and with a damping scheme to reduce coherent betatron oscillations,

5. Improving the storage ring field uniformity and the measurement and calibration system,

6. Increasing the detector segmentation to reduce the instantaneous rate.

Items $1-3$ will be realized by a clever use of the existing FNAL accelerator complex. A single 8-GeV Booster batch will be injected into the Recycler, where it will be subdivided into 4 bunches; 6 of the 20 batches per $15 \mathrm{~Hz}$ Booster cycle provide 24 individual bunches of $10^{12}$ protons. Each bunch is directed to the existing antiproton target to produce $3.1 \mathrm{GeV} / \mathrm{c}$ positive pions, which will be focussed by the existing lithium lens system and a new pulsed dipole magnet into the AP2 transfer line. The quadrupole density in the 270-m long AP2 line will be increased to reduce the beta function and consequently capture and transport a high fraction of forward-decay muons. The muon beam will go around the Debuncher ring for nearly one full turn, then be extracted into the existing AP3 channel back toward the AP0 building. A new, short, transfer line will direct the beam into the storage ring through a new open-ended inflector magnet. The storage ring will be located in a new building near AP0 where ample cryo and power services are available nearby. In subsection IV C we discuss how the background from the injection flash will be reduced.

Item 4 will be approached by optimization of the storage ring kicker pulse and possible implementation of a damping scheme to reduce muon betatron oscillations. We discuss this complex subject in Appendix B.

Item 5 involves the magnetic field. As the ring is rebuilt for FNAL, an extra degree of effort will be required to shim the field to a higher level of uniformity. To meet the stringent demands of the systematic error goal for this measurement, some R\&D projects will be required, as detailed in the Field section. Additionally, an improved positron traceback system of detectors will be required to image the beam for folding together with the field maps to obtain the muon-averaged magnetic field. 
Finally, for item 6 , we detail a plan to segment the positron detector system to reduce pileup. Further, a new electronics and DAQ system will be capable of storing events having lower electron energies.

\section{The Expected Flash at FNAL}

At BNL, one key limitation to simply increasing the rate was the hadronic flash that was induced by pions entering the ring at injection. These pions crashed into the detectors and the magnet steel, producing neutrons, which thermalized with a time constant close to the time-dilated muon lifetime. In the calorimeters, neutron-capture gamma rays led to a slowly decaying baseline shift in the light reaching the PMTs. Positron signals then had to be extracted from above a time-shifting baseline. The baseline shift affected a number of systematic errors, which we expect to be largely absent in the new experiment. The prompt flash-the short burst of direct background in the detectors at injection-required us to gate off all detectors during injection and turn them back on some $5-15 \mu$ s later. The slow neutron capture produced a baseline shift that was evident for 10 's of $\mu$ s afterward. To estimate the flash for FNAL, we consider four differences in operation between BNL and FNAL.

- At BNL, the proton intensity per storage ring fill was $\sim 4 \times 10^{12}$. At FNAL, it will be $1 \times 10^{12}$ (intensity factor $=4$ ).

- At BNL, the proton beam energy was $24 \mathrm{GeV}$; at FNAL it is $8 \mathrm{GeV}$. The pion production yield is approximately 2.5 times higher at BNL. (pion-yield factor $=2.5$ ).

- The FNAL pion decay beamline will be $\sim 800 \mathrm{~m}$ longer compared to BNL. With a decay length for $3.1 \mathrm{GeV} / \mathrm{c}$ pions of $173 \mathrm{~m}$, the difference represents a reduction by a factor of 100 in undecayed pions at FNAL compared to at BNL (decay factor $=100$ ).

- To improve the ratio of injected muons to pions at BNL, the ratio of upstream pionselection momentum to final muon magic momentum, $\left(P_{\pi /} / P_{\mu}\right)$, was set to 1.017. This reduced the pion flux by 50 (and the muon flux by $\sim 3-4$ ). For FNAL the ideal ratio of $P_{\pi} / P_{\mu}=1.005$ will be used. The asymmetric $B N L$ setting reduced the transmitted undecayed pions compared to anticipated FNAL equivalent settings $\left(P_{\pi} / P_{\mu}\right.$ factor $=$ $0.02)$. 
The flash is based on the pion flux entering the ring per storage ring fill (not on the stored muon rate). The four factors above intensity, pion yield, decay, $P_{\pi} / P_{\mu}-$ multiply: $4 \times$ $2.5 \times 100 \times 0.02=20$. This implies that the flash will be 20 times smaller at FNAL in the new experiment compared to BNL.

\section{Event Rate and POT Request Calculation}

A preliminary estimate of the event rate and therefore total proton-on-target (POT) request required for acquiring the $1.8 \times 10^{11}$ events is outlined in Table IV. Up to the target, we used known factors for proton beam delivery as outlined in this proposal. A pion production calculation using MARS was made to estimate the number of $3.1 \mathrm{GeV} / \mathrm{c}$ pions emitted into the accepted phase space of the AP2 line. From this point, a conservative approach was to compare known factors between the muon capture and transmission at FNAL to those same factors at BNL. Many of the factors are relatively trivial to compute, while others rely on our detailed Decay Turtle simulations of the BNL lattice and modifications of this lattice for FNAL. We are in the process of a complete end-to-end calculation of the beamline, but this work will take additional time. In the comparison to BNL approach, we find the important increase of stored muons per incident proton of 11.5; whereas, we require a factor of at least 6 for an experiment that can be done in less than 2 years. We use the factor of 6 in our beam estimates, thus introducing a "beam-time contingency" factor of nearly $100 \%$ from the beginning. Experience from E821 suggests that $4-6$ weeks of setup time with beam will be required before "good" data are obtained. We also assume that $66 \%$ of the data taking period will result in the final statistics - this allows for regular magnet mapping intervals, systematic runs, calibration runs, and normal experimental downtime. The origin of each factor in Table IV, is explained in a series of notes following the Table. 
TABLE IV: Event rate calculation using known factors and a comparison to the realized stored muon fraction at BNL.

\begin{tabular}{|l|r|r|r|}
\hline Item & Factor & Net & Note \\
\hline Booster cycle $-15 \mathrm{~Hz}$ operation & $1.33 \mathrm{~s} / \mathrm{cycle}$ & $0.75 \mathrm{~Hz}$ & 1 \\
Batches to $(g-2)$ & 6 & $4.51 \mathrm{~Hz}$ & 2 \\
Protons on target & $4 \times 10^{12} p /$ batch & $1.80 \times 10^{13} \mathrm{p} / \mathrm{s}$ & 3 \\
Bunches (each bunch provides 1 fill of the ring) & $4 /$ batch & $18 \mathrm{fils} / \mathrm{s}$ & 4 \\
BNL stored muons per proton & $1 \times 10^{-9} \mu / p$ & $1000 \mu / \mathrm{Tp}$ & 5 \\
Minimum stored $\mu / p$ improvement FNAL vs. BNL & 6.0 & $6000 \mu / \mathrm{Tp}$ & 6 \\
Positrons with $t>30 \mu$ and $E>1.8 \mathrm{GeV}$ & $10 \%$ & $603 \mathrm{e}^{+} / \mathrm{fill}$ & 7 \\
DAQ / Expt. production and uptime & $66 \%$ & & 8 \\
Time to collect $1.8 \times 10^{11}$ events $\left(2 \times 10^{7} \mathrm{~s} / \mathrm{y}\right)$ & & 1.25 years & 9 \\
Commissioning time & & 0.1 years & 10 \\
FNAL running years & & 1.35 years & 11 \\
\hline Total Protons on Target & $4 \times 10^{20}$ POT & 12 \\
\hline
\end{tabular}

Notes explaining entries in Table IV:

1. $15 \mathrm{~Hz}$ Booster operation is assumed.

2. Neutrino program uses 12 out of 20 batches; 8 out of 20 are in principle available, but 6 should be clean for use by the muon program. Batches are injected into the Recycler with $66 \mathrm{~ms}$ spacing.

3. Standard expected proton intensity per batch.

4. Subdivision in Recycler of each batch into 4 "bunches" with roughly equal intensity. Each is extracted separately with $\sim 12 \mathrm{~ms}$ spacing and each initiates a storage ring "fill."

5. Measured stored muon fraction per 24 -GeV proton on target at BNL per $10^{12} p$ (Tp). This number folds up individual factors including the inflector transmission and the storage ring kicker efficiency.

6. The improvement is done comparing to the known situation at BNL. We arrive at the following factors: $\times 0.4$ for the reduced pion yield; $\times 1.8$ for the AP2 line with smaller 
beta function; $\times 2$ for the longer decay channel; $\times 3$ for the forward decay optimal muon tune; $\times 1.33$ for opening up the pion momentum acceptance; $\times 2$ for the open inflector and improved kicker $=11.5$. We use a factor of 6 to be very conservative. See details in Section $V E$.

7. Product of $16 \%$ acceptance from GEANT Monte Carlo for decay positrons having energy greater than $1.8 \mathrm{GeV}$ and muon population reduction from injection to the expected fit start time at $t=30 \mu \mathrm{s}$.

8. Expected global uptime fraction, which includes time for magnetic field mapping, systematic error studies, calibration runs, and normal equipment malfunction.

9. With above factors, and using $2 \times 10^{7} \mathrm{~s}$ of delivered beam per FNAL year.

10. Estimate of setup time with beam on.

11. Total running.

12. Request POT under above conditions. Actual needs will depend on completed beam design. 


\section{BEAM PREPARATION}

The Proton Plan and the NOvA Project at Fermilab will allow the Main Injector to run with a 1.333 s cycle time for its neutrino program (NuMI), with twelve batches of beam from the Booster being accumulated in the Recycler and single-turn injected at the beginning of the MI cycle. Thus, there remain eight Booster cycles during each MI period that could in principle be used for an $8 \mathrm{GeV}$ (kinetic energy)[103] beam experimental program. Under the Proton Plan[58], the maximum average Booster repetition rate has been increased from roughly $2.5 \mathrm{~Hz}$ to $9 \mathrm{~Hz}$. While not required for the NuMI program, a further upgrade to the Booster RF system remains necessary to allow the Booster to run at its maximum rate of 15 $\mathrm{Hz}$. This upgrade is required for any use of the Booster for programs complementary to the neutrino program and in subsequent subsections we will assume this has been performed. Additionally the per cycle intensity may be greater with these upgrades, but for purposes of this discussion we will use a typical $4 \times 10^{12}$ protons ( $4 \mathrm{Tp}$ ) per Booster batch.

Beam for the $\mathrm{g}-2$ experiment is to be transferred directly into the Recycler ring from the Booster and out of the Recycler into the P1 transport line. At the moment these functions are performed directly to and from the Main Injector. However, the NOvA project also requires injection into the Recycler from the Booster, and so it will be assumed for our discussion that this functionality has been achieved at the end of that project. Extraction from the Recycler and delivery to the P1 beam line is required, with costs similar to the aforementioned injection system.

The Debuncher, Accumulator, and Recycler rings all have equipment installed to perform stochastic cooling (and, in the Recycler, electron cooling) which can and should be removed to generate less aperture restrictions for the high intensity operations of any $8 \mathrm{GeV}$ experimental program.

Particle losses in the Booster are currently observed over a 100 s running average as detected by the beam loss monitor system and limit the beam delivered by the synchrotron to about $1.6 \times 10^{17}$ protons/hour. Comparatively, $15 \mathrm{~Hz}$ operation at $4 \mathrm{Tp}$ per batch would produce roughly $2.2 \times 10^{17}$ protons per hour. It is expected that the new magnetic corrector system, the installation of which will be completed in 2009 under the Proton Plan, will allow for this increased intensity under $15 \mathrm{~Hz}$ operation.

Table $\mathrm{V}$ outlines the scope of the work to be performed for implementation of the New 
$(g-2)$ Experiment at Fermilab.

TABLE V: Scope of accelerator system modifications required of accelerator systems.

\begin{tabular}{|c|c|l|}
\hline Accel/BmL & System & Note \\
\hline Booster & RF & upgrade to 15 Hz operation \\
Recycler & inj line & from MI-8 to RR \\
Recycler & ext line & from RR to P-1 line \\
Recycler & cooling & remove stoch/e- cooling systems \\
Recycler & ext line & extraction kicker \\
Recycler & RF & system move from MI, upgrades \\
AP0 & target station & possible new optics, lens upgrades \\
Expt Hall & building & new construction \\
Expt Hall & cryo & tie in with Tevatron system \\
transf. lines & Rad. Safety & mitigation near new building \\
Rings, transf. lines & Instr/Controls & possible BPM upgrade \\
\hline
\end{tabular}

\section{A. Meeting the Experimental Requirements}

The $(g-2)$ experiment requires $3.09 \mathrm{GeV} / \mathrm{c}$ muons injected into an existing muon storage ring that would be relocated from Brookhaven National Laboratory to Fermilab. The muon storage ring is $7.1 \mathrm{~m}$ in radius, giving a revolution time of $149 \mathrm{~ns}$. To account for the injection kicker, the beam pulses need to have RMS lengths of about 50 ns or less. These pulses should be separated on the scale of about $10 \mathrm{~ms}$ for the muons to decay in the ring and data to be recorded prior to the next injection. To obtain as pure a muon beam as possible entering the storage ring, the experiment would like a decay channel corresponding to several pion decay lengths, where $c \beta \gamma \tau_{\pi}=173 \mathrm{~m}$. Present understanding of the pion yield, the transfer line acceptance, and the muon storage fraction support the idea that the $21 \times$ more statistics can be obtained in less then 2 years of running. 
To meet the above requirements it is envisioned that six Booster batches every MI cycle can be sent to the experiment for an average rate of $6 / 20 \times 4 \mathrm{Tp} \times 15 / \mathrm{s}=18 \mathrm{Tp} / \mathrm{s}$. This yields the required total protons on target. Each batch of $53 \mathrm{Mhz}$ bunches from the Booster would be sent to the Recycler and formed into four bunches for delivery to the experiment. Using existing RF systems, possibly supplemented with like-kind components, the four bunches can be formed to meet the demands of the $(g-2)$ ring. The re-bunching process takes approximately $30 \mathrm{~ms}$, and the four bunches would then be delivered to the experiment one at a time spaced by $12 \mathrm{~ms}$. Thus, the last bunch is extracted just within the $66.7 \mathrm{~ms}$ Booster cycle. The remaining two Booster cycles, before and after this process, allow for pre-pulsing of fast devices prior to the change between NuMI and "muon" cycles. (If this is deemed unnecessary, then eight rather than six Booster cycles could feed the experiment during each MI cycle.) Figure 16 shows the proposed time line of events during MI operation.

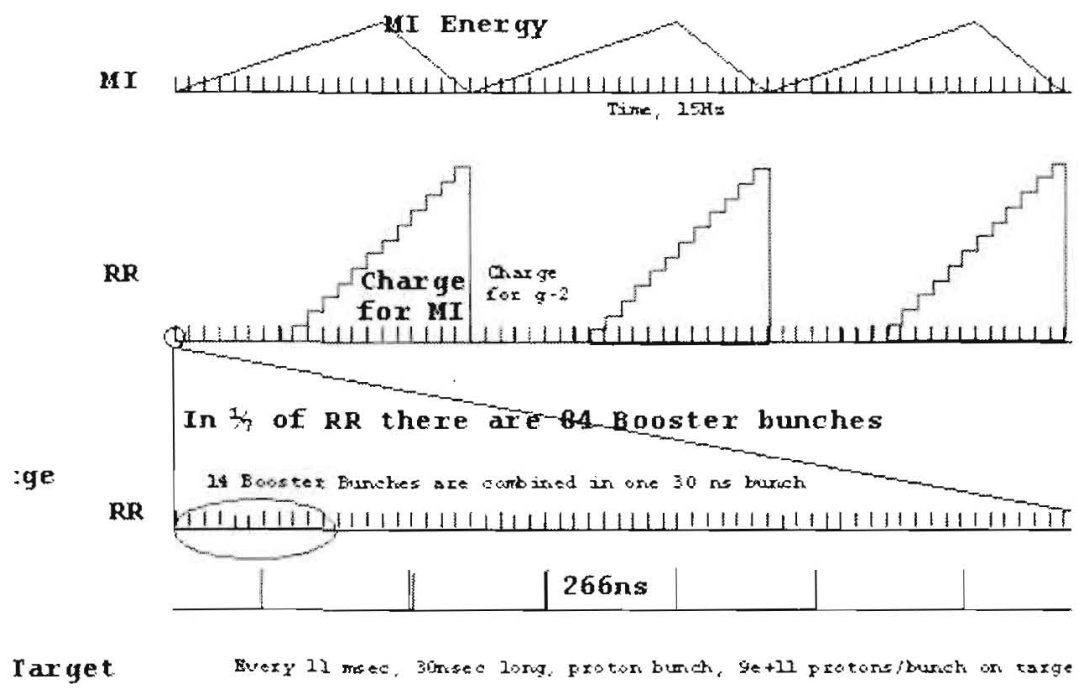

FIG. 16: Timing diagram for the proposed g-2 operation.

Once extracted from the Recycler a bunch is sent toward the existing, though possibly modified, antiproton target station for $\sim 3.11 \mathrm{GeV} / \mathrm{c}$ pion production. A "boomerang" approach utilizing the Debuncher and Accumulator rings can be used as a delay line allowing for pion to muon decay, assuming a final location of the g-2 ring in the vicinity of the 
production target. A schematic of the beam line system is presented in Figure 17. The total

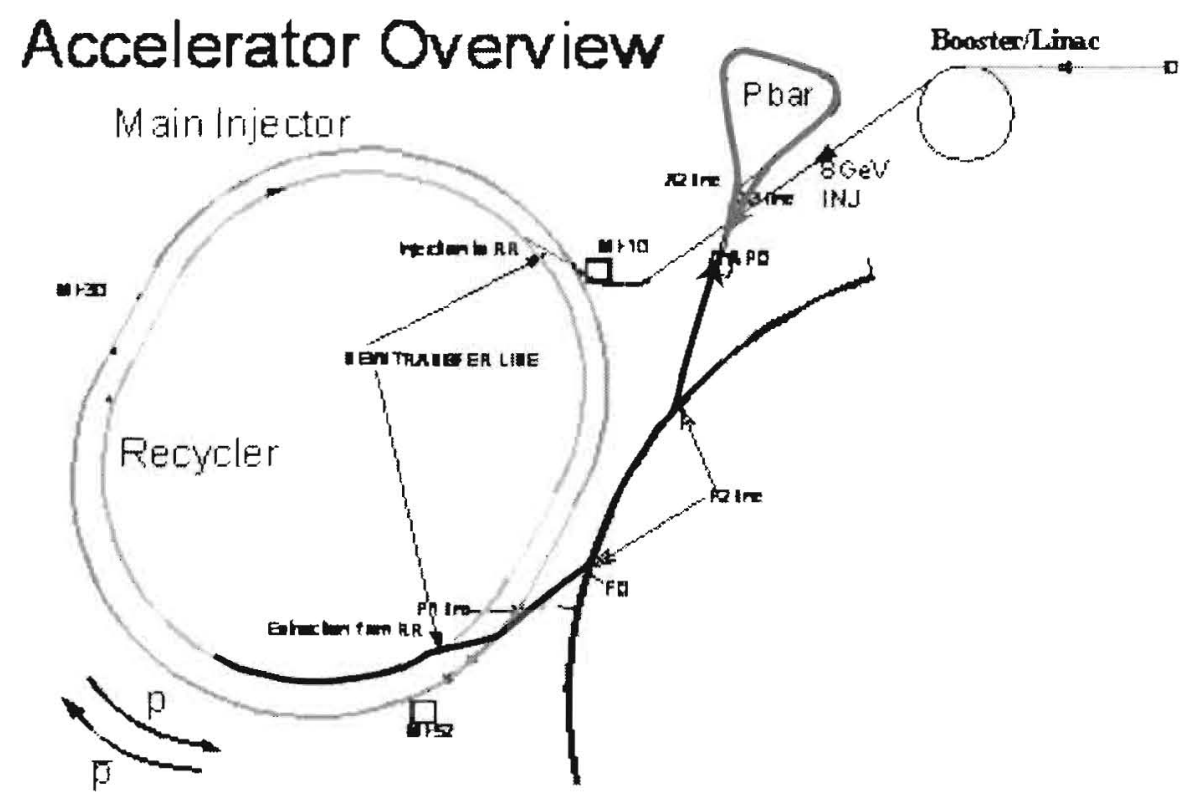

FIG. 17: Bearn transport scheme for g-2 operation. Beam is prepared in the Recycler, exits via the P1 line, passes through the Tevatron tunnel into the AP1 beam line, and to the AP0 target area. (Blue curve.) Pions, decaying to muons, are transported frorn the target through the AP2 line, once around the "pbar" rings (Debuncher/Accumulator) and back toward the experimental hall near AP0 via the AP3 beam line. (Thick red curve.)

length of the decay line would be $\sim 900 \mathrm{~m}$. To obtain even further purity of the muon beam, multiple revolutions in the Debuncher or Accumulator rings could be considered, perhaps as an upgrade to the program. This upgrade would require the development of an appropriate kicker system and is not included in this first design iteration. The $900 \mathrm{~m}$ decay length, however, is already a large improvement over the original layout at BNL.

\section{B. Bunch Formation}

The major proton beam preparation will be performed in the Recycler ring. A broadband RF system like that already installed in the Recycler would be used, except twice the voltage may be required. The $2.5 \mathrm{MHz}\left(\max . V_{r f}=60 \mathrm{kV}\right)$ and $5 \mathrm{MHz}\left(\max . V_{r f}=15 \mathrm{kV}\right) \mathrm{RF}$ 
systems that presently reside in the MI would be relocated to the Recycler. Upgrades to increase their maximum voltages by roughly 10-30\% may be required. All of these upgrades are assumed for the cost estimate.

As described in [59], the bunching scheme is to use a four period sawtooth wave form across the Booster batch produced by the broadband RF system to break the batch into four segments and rotate them in phase space sufficiently that they can be captured cleanly in a linearized bucket provided by the resonant RF. Each of the four resulting bunches has an RMS length of $\sim 50 \mathrm{~ns}$. The first bunch is extracted immediately and the latter three are extracted sequentially at half periods of the synchrotron oscillation. The beam loading of the resonant cavities will be considerable, and further details need to be considered. It is plausible to expect that a feedforward system can be developed without serious difficulty. A combination of feedback with feed forward is potentially better yet, but feedforward will be required with or without feedback. Figure 18 shows the resulting beam structure in the

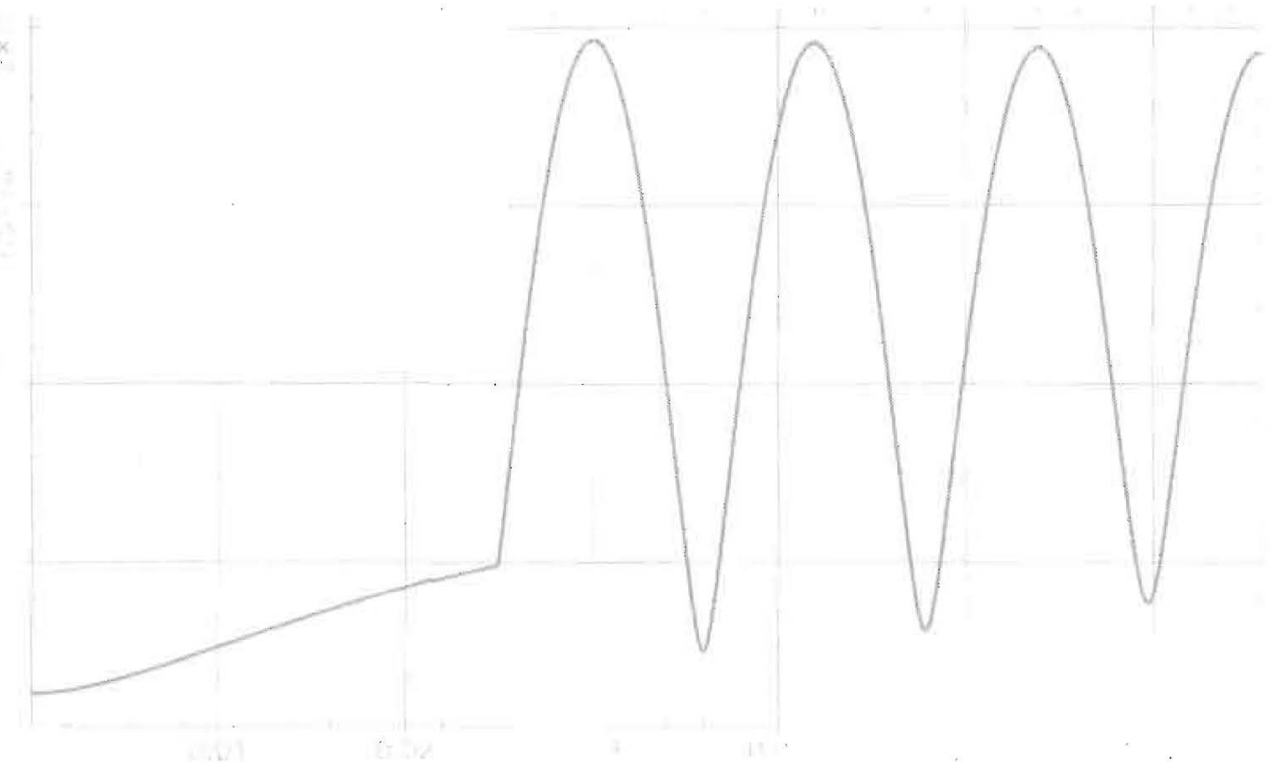

FIG. 18: Resulting relative momentum spread $(\Delta p / p)$ vs. time in seconds following injection into the Recycler. After an initial phase using the broadband RF system, beam is captured into four buckes. The beam rotates within the four buckets with period $12 \mathrm{~ms}$ and is extracted one-by-one as the momentum spread reaches its peak (pulse length is at its shortest). 
Recycler if the beam were not extracted. The plan would be to extract one pulse at a time, every $12 \mathrm{~ms}$, when the bunches are at their narrowest time extent ( $4 \sigma$ widths of 38-58 ns). The four bunches would be separated by roughly $400 \mathrm{~ns}$ center-to-center. For the sequence shown, the RF systems require voltages of $4 \mathrm{kV}$ (broadband), $80 \mathrm{kV}(2.5 \mathrm{MHz}$ ), and $16 \mathrm{kV}$ (5.0 MHz). A longitudinal emittance of $0.07 \mathrm{eV}-\mathrm{s}$ per $53 \mathrm{MHz}$ Booster bunch was assumed.

\section{Beam Delivery and Transfer}

Following the beam trajectory starting with extraction from the Booster, we see that the proton beam needs to be injected into the Recycler from the MI- 8 beam line at the MI-10 region of the Main Injector tunnel. This maneuver will be facilitated through the NOvA project, which requires the same injection procedure. Once prepared with the RF systems as described above, the beam will need to be extracted from the Recycler and injected into the P1 beam line. The extraction location is at the MI-52 tunnel location, where the Main Injector ties into this same beam line. (See Figure 17.) The P1 beam line is used to deliver $8 \mathrm{GeV}$ antiprotons from the Accumulator into the Main Injector (and on into the Recycler) in the reverse direction. During the g-2 operation, however, the Main Injector will contain beam destined for NuMI and so this region will need to be modified in almost exactly the same way as MI-10 to transport protons directly into the P1 line from the Recycler.

An appropriate kicker system will also be required for this region to extract one-byone the four proton bunches from the Recycler. The four bunches will be separated by approximately $200 \mathrm{~ns}$, so the kicker must rise in $\sim 180 \mathrm{~ns}$ and have a flat top of $\sim 50 \mathrm{~ns}$. The Recycler has a circumference seven times that of the Booster, and only one Booster batch will be injected at a time. Thus, the last proton bunch of the four will be separated from the first by about $8.6 \mu$ s or more. The kicker can then have a fall time on the order of $5 \mu s$, and must be pulsed 4 times separated by $10 \mathrm{~ms}$ within a Booster cycle. This operation is repeated 6 times every $1.33 \mathrm{~s}$ MI cycle.

From the entrance of the P1 line through the Tevatron injection Lambertson (which is kept off during this operation) the beam is directed through the P2 line (physically located in the Tevatron tunnel) and into the AP1 line toward the AP0 target hall. Again, since this system is run at $8 \mathrm{GeV}$ for antiproton operations, no modifications are required for beam transport in g-2 operations. After targeting, which is discussed in the next subsection, 
$3.1 \mathrm{GeV} / \mathrm{c}$ pions are collected into the AP2 line which is "retuned" to operate at $3.1 \mathrm{GeV} / \mathrm{c}$ rather than today's $8.89 \mathrm{GeV} / \mathrm{c}$ antiproton operation.

To obtain a long decay channel for the pions off the target, the beam is transported through the AP2 line, into the Debuncher ring, and, through a new short transfer line, into the AP3 line, and directed back toward AP0. (See Figure 17 again.) An alternative option being considered is to transfer the beam from the Debuncher into the Accumulator using existing lines; however, the present aperture restrictions in this simpler mode require further study. As this will be the only use of these rings, kicker magnets will not be required in this configuration, and the rings will be "partially powered" using only those magnet strings required to perform the "boomerang." Either corrector magnets or DC powered trim magnets will be used in place of kickers to perform the injection/extraction between the partially powered rings and associated beam lines. It is currently envisioned that the g- 2 ring will be located on the surface near the AP0 service building as indicated in Figure 19. The AP3 beam line will be modified to "punch through" the ceiling of the tunnel enclosure

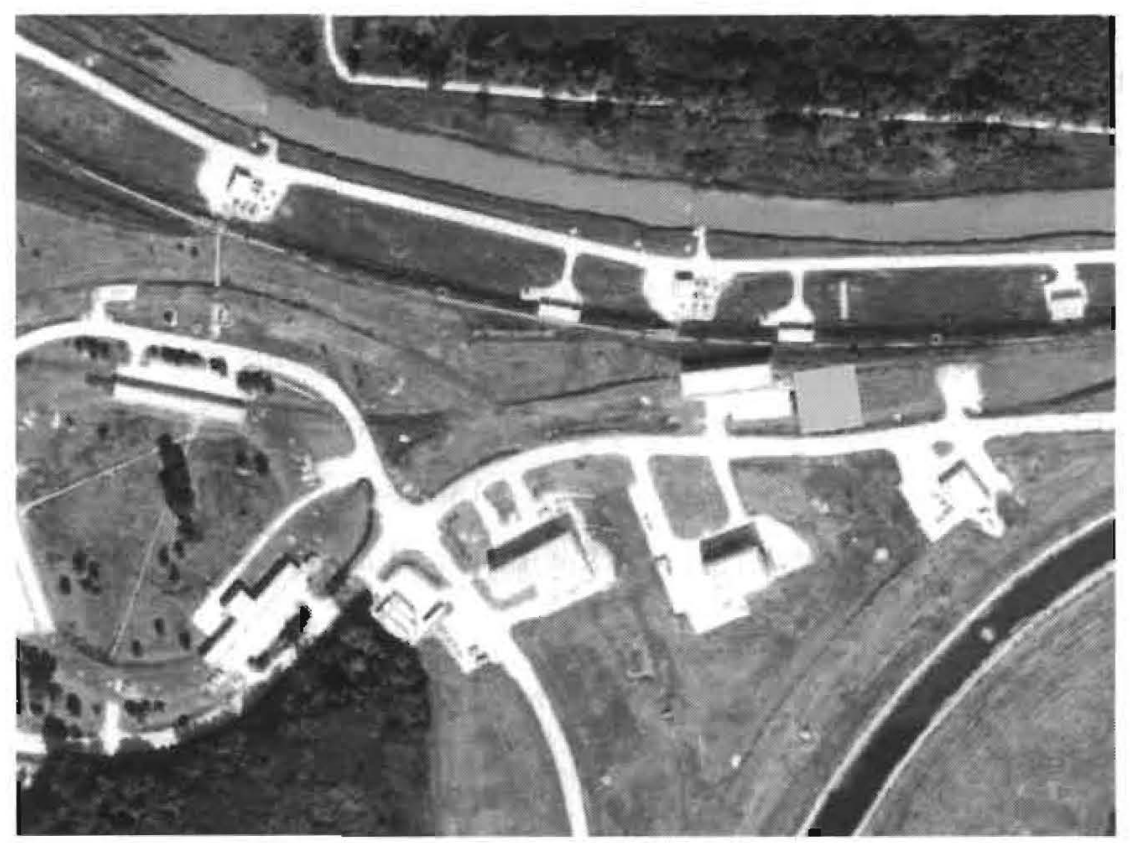

FIG. 19: Proposed location of the new $(g-2)$ experimental hall (yellow).

and up into the g-2 ring.

As can be seen, little modifications are required of existing beam lines to perform the beam transport all the way from the Booster to the $\mathrm{g}-2$ ring. The end of the line and connection 
to the experimental ring requires design, but should be straightforward. It should also be reiterated that the Debuncher, Accumulator, and Recycler rings all have stochastic cooling (and, in the Recycler, electron cooling) equipment which should be removed to mitigate aperture restrictions.

\section{Target Station, Pion Capture and Decay}

Various options are being explored at this time for meeting the targeting requirements of the experiment, and much headway is being made. Using the existing APO target moreor-less "as is" seems to be the most straightforward approach. The present system is used for selecting $8.9 \mathrm{GeV} / \mathrm{c}$ antiprotons from a $120 \mathrm{GeV} / \mathrm{c}$ primary proton beam. For $\mathrm{g}-2$ one would select $\sim 3.1 \mathrm{GeV} / \mathrm{c}$ pions from $8.9 \mathrm{GeV} / \mathrm{c}$ primary protons by re-tuning the beam lines upstream and downstream of the target. The major issues with this particular scenario is the use of the existing Lithium lens, critical for antiproton production at $120 \mathrm{GeV} / \mathrm{c}$, and the use of the existing pulsed bending magnet just downstream of the lens. Both of these devices are pulsed once every $2.2 \mathrm{~s}$ during antiproton operation. Options for re-configuring these devices and their power supplies into a useful operational mode, such as to pulse every $12 \mathrm{~ms}$ or to generate a single pulse with a flat top of about $40 \mathrm{~ms}$, with appropriate reduced currents, are being investigated. Use of the existing Lithium lens may be feasible, but other optical solutions could be considered if necessary, such as a quadrupole triplet system. It is likely, however, that the existing pulsed dipole magnet system will need to be upgraded to match the increased repetition rate. The APO target vault area allows for modular reconfiguration of such devices, and thus various options can be explored should the existing systems need renovation.

\section{E. Toward a Beam Rate Calculation}

The experiment at FNAL requires at least a 6 fold increase of the number of stored muons per $8 \mathrm{GeV}$ proton compared to that obtained by $\mathrm{E} 821$ for $24 \mathrm{GeV}$ protons. Table VI summarizes the main gain factors of the New $(g-2)$ Experiment relative to E821 and their origins. More details of the beam concept are addressed in Appendix $A$. These estimates are preliminary and the proposed R\&D plan foresees detailed end-to-end simulations as well 
as beam tests to corroborate these numbers. Additional improvements, due to a faster ring injection kicker or tighter proton focusing on the production target, are under investigation. Thus, the required gain factor of 6 can likely be exceeded, but we conservatively assume this factor as a planning baseline for this proposal.

\begin{tabular}{lccc}
\hline parameter & BNL & FNAL & gain factor FNAL/BNL \\
\hline$Y_{\pi}$ pion/p into channel acceptance & $\approx 2.7 \mathrm{E}-5$ & $\approx 1.1 \mathrm{E}-5$ & 0.4 \\
$\mathrm{~L}$ decay channel length & $88 \mathrm{~m}$ & $900 \mathrm{~m}$ & 2 \\
decay angle in lab system & $3.8 \pm 0.5 \mathrm{mr}$ & forward & 3 \\
$\delta p_{\pi} / p_{\pi}$ pion momentum band & $\pm 0.5 \%$ & $\pm 2 \%$ & 1.33 \\
FODO lattice spacing & $6.2 \mathrm{~m}$ & $3.25 \mathrm{~m}$ & 1.8 \\
inflector & closed end & open end & 2 \\
\hline total & & & 11.5 \\
\hline
\end{tabular}

TABLE VI: Parameters for E821 and the New $(g-2)$ Experiment beamline and their relative effect on the stored muons per proton fraction. Pion yield $Y_{\pi}$ given for pion momentum bin $p_{\pi}=$ $3.11 \mathrm{GeV} / \mathrm{c} \pm 0.5 \%$

\section{F. Experimental Facility}

A first pass look at an experimental building was performed at Fermilab. [60] The building would be approximately $80 \mathrm{ft} \times 80 \mathrm{ft}$ and includes a full-span 40 ton bridge crane. Other details of the cost exercise may be found in [60]. The building is large enough to enclose the g-2 ring as well as associated electronics and counting room. A schematic of the building location and adjacent parking area are found in Figure 20.

The cryogenic needs of the experiment can be met by the Tevatron accelerator cryogenics system with some modifications and additional transfer line work. The Tevatron is located only about $50 \mathrm{ft}$ away from the APO service building; and is expected to be in $80^{\circ} \mathrm{K}$ standby mode during the time span of the experiment. Additionally, it is assumed that the Tevatron F2 magnet string is allowed to be warmed up to room temperature during this time, freeing up the refrigeration system at $\mathrm{F} 2$ to be used for $\mathrm{g}-2$. A cost estimate of the required modifications has been performed and documented.[61] 


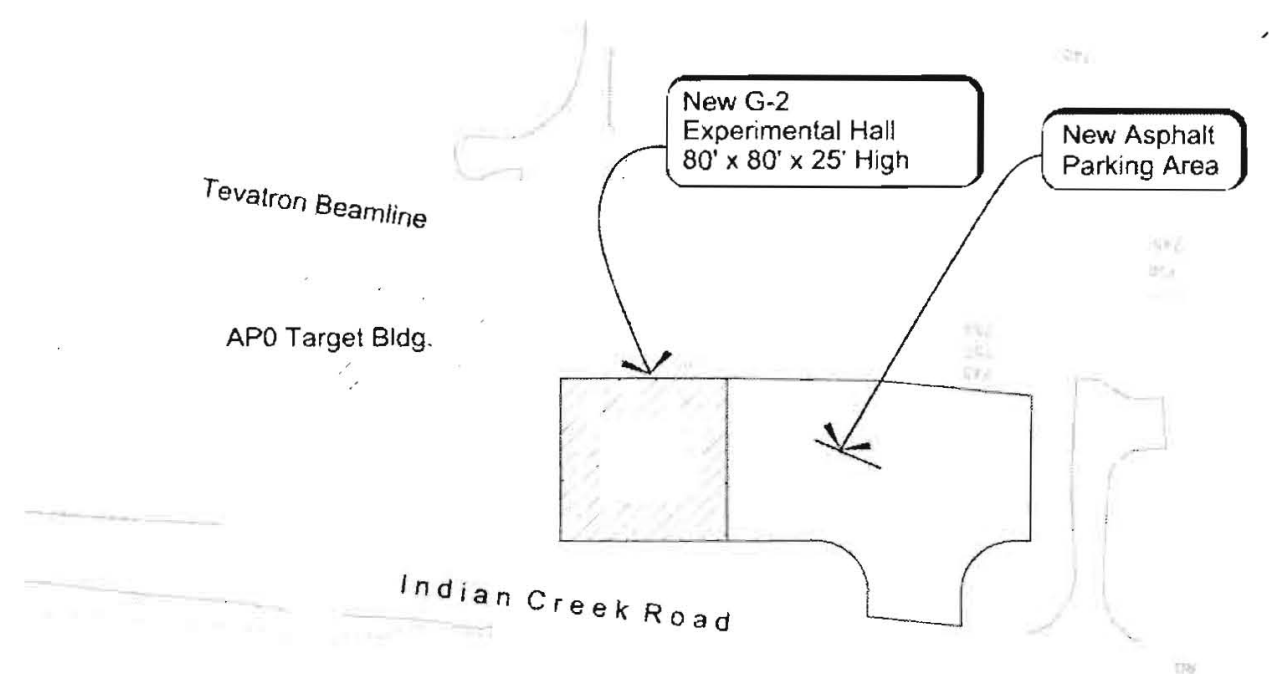

FIG. 20: Location plan of the new g-2 experimental hall.

The exact location of the building south of APO will be determined by the final design of its connection with the beam transport line. The beam line will need to emerge vertically from the tunnel containing the AP3 line and make a roughly $90^{\circ}$ horizontal bend into the experiment building.

\section{G. Environmental Assessment}

As noted previously, the average particle delivery rate to the g-2 target would be $18 \mathrm{Tp} / \mathrm{sec}$. At $8 \mathrm{GeV}$ kinetic energy per proton, this translates to approximately $27 \mathrm{~kW}$ beam power onto the target station. Present day antiproton production operation utilizes two Booster batches of $4 \mathrm{Tp}$ every $2.2 \mathrm{sec}$ at a particle energy of $120 \mathrm{GeV}$, which corresponds to approximately $67 \mathrm{~kW}$ beam power onto target. Thus, the activation of the target hall and beam lines leading up to it is expected to be well below present day levels. This should also be expected of the beam delivery from the target into and out of the rings and back to the AP0 region through the existing beam lines since this will be performed as a single-pass beam transport using DC magnetic elements. Once designed, the final layout of the connecting region between the AP3 beam line and a new g-2 experimental hall will need 
to be assessed for appropriate shielding. While further work will be needed to validate the environmental impact of the new use of these facilities for $\mathrm{g}-2$, as well as for the experimental building itself, this is seen as a straightforward effort.

\section{H. Opening the Inflector Ends}

The original superconducting inflector design for BNL E821 included two options for the ends: open or closed, see Fig. 21. Both versions were built in $0.5 \mathrm{~m}$ long prototype form, but only the closed-end version was built at full scale $(1.7 \mathrm{~m}$ length) and used in the experiment $[62,63]$. The closed infiector was selected because of its simpler construction and was thought to be more stable against Lorentz forces. Further, the closed-end inflector has a smaller fringe field that could be more easily shielded from the storage ring field seen by the muons. On the downside, beam transport studies show that multiple scattering and energy loss in the closed end reduce the transmission of muons that store in the ring by a factor of 2 .

The stability of the open-ended coil configuration was demonstrated at full current in a $1.5 \mathrm{~T}$ external magnetic field. Based on our measurements [63], the added leakage field from the open end can be excluded from the storage region by a passive superconducting sheet.

A factor of 2 increase in muon flux is expected from opening the ends. Much of the investment in engineering for this device has already been made and custom tooling necessary to construct the magnet exists. The inflector is discussed in detail in an appendix.

\section{Accelerator $R \& D$}

As of this writing the major items requiring attention include:

- Targeting and Pion Flux: The target optics needs to be verified and/or re-designed if necessary, including the possible use of the lithium lens, and the final target material chosen or verified. The possible use of the existing pulsed dipole magnet in the target hall needs to be addressed as well and options identified should this magnet not meet specifications. Headway is being made quickly on understanding the expected pion flux from targeting of $8 \mathrm{GeV}$ kinetic energy protons. The associated production acceptance 


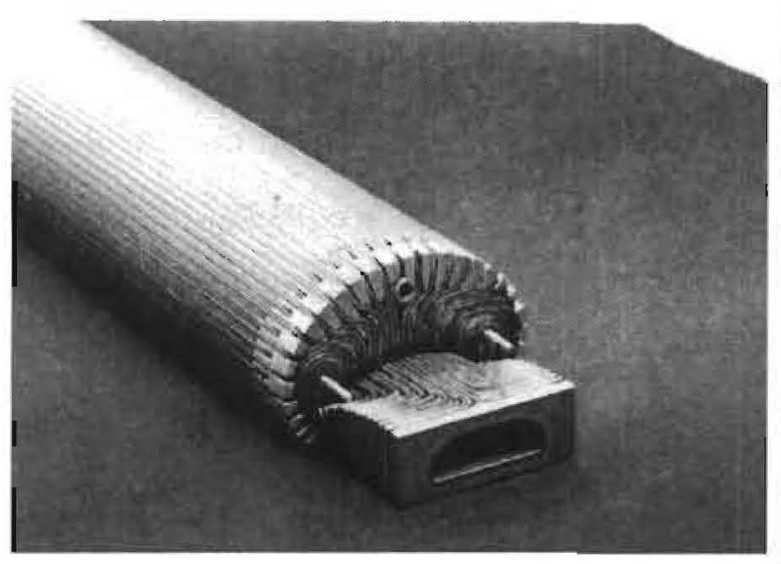

(a) Open End

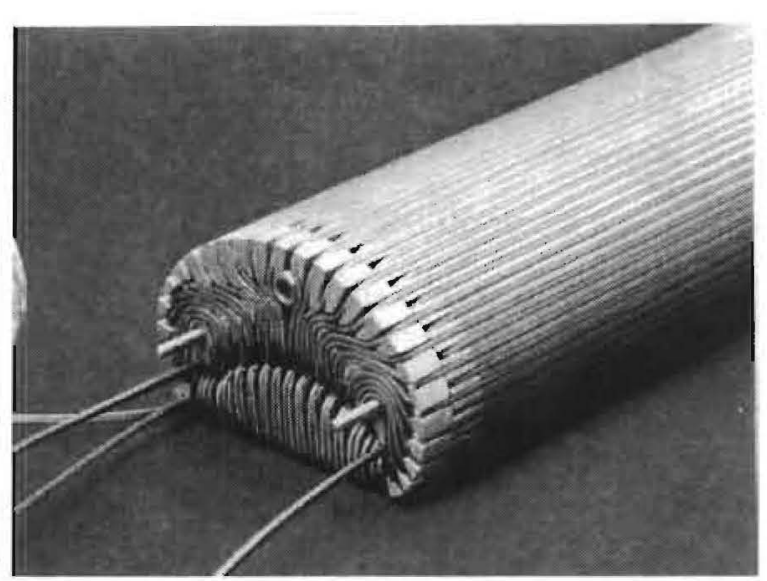

(b) Closed End

FIG. 21: Photos of the open- and closed-end inflector prototype.

into the transport line is being carefully examined, modeled, and documented. This effort will continue with high priority.

- Intensity Limitations: Studies should be performed on the intensity limitations of the Recycler, for example the impedances expected to be present during g-2 operation. While many of today's electron and stochastic beam cooling components can be removed, the addition of new RF systems will create new sources of impedance that need to be examined. The $\mathrm{NO} \nu \mathrm{A}$ program, for instance, is expecting a low impedance system to meet its intensity requirements, and any modifications must be consistent with this expectation.

- Radiation Safety: Assessment needs to begin soon on the required improvements to radiation shielding or other mitigation for the increased particle flux through the antiproton source beam lines and synchrotron tunnels.

- Bunch Formation: Optimization of the bunch formation in the Recycler and final definition and specification of the RF requirements need to be completed.

- Final Transport: The final stage of beam transport from the AP3 beam line up and into the g-2 ring in the experimental hall needs to be designed and properly costed. 


\section{J. Muon Storage Ring Magnet}

The muon storage ring $[64]$ is a superferric " $\mathrm{C}$ "-shaped magnet, $7.112 \mathrm{~m}$ in central orbit radius, and open on the inside to permit the decay electrons to curl inward to the detectors (Fig. 22). A $5 \mathrm{~V}$ power supply drives a $5177 \mathrm{~A}$ current in the three $\mathrm{NbTi} / \mathrm{Cu}$ superconducting coils. Feedback to the power supply from the NMR field measurements maintains the field stability to several ppm. The field is designed to be vertical and uniform at a central value of $1.4513 \mathrm{~T}$. High-quality steel, having a maximum of 0.08 percent carbon, is used in the yoke. Low-carbon steel is used for the poles primarily because the fabrication process of continuous cast steel greatly minimizes impurities such as inclusions of ferritic or other extraneous material and air bubbles. An air gap between the yoke and the higher quality pole pieces decouples the field in the storage region from non-uniformities in the yoke. Steel wedge shims are placed in the air gap. Eighty low-current surface correction coils go around the ring on the pole piece faces for active trimming of the field. The opening between the pole faces is $180 \mathrm{~mm}$ and the storage region is $90 \mathrm{~mm}$ in diameter. A vertical cross section of the storage ring illustrating some of these key features is shown in Fig. 23. Selected storage ring parameters are listed in Table VII.

Attaining high field uniformity requires a series of passive shimming adjustments, starting far from and then proceeding towards the storage region. First the twelve upper- and loweryoke adjustment plates are shimmed by placing precision spacers between them and the yoke steel, modifying the air gap. Next the 1000 wedge shims in the yoke pole-piece air gap are adjusted. With a wedge angle of $50 \mathrm{mrad}$, adjusting the wedge position radially by $1 \mathrm{~mm}$ changes the thickness of iron at the center of the storage aperture by $50 \mu \mathrm{m}$. The wedge angle is set to compensate the quadrupole component, and radial adjustments of the wedge and other changes to the air gap are used to shim the local dipole field. The local sextupole field is minimized by changing the thickness of the 144 edge shims, which sit on the inner and outer radial edges of the pole faces. Higher moments, largely uniform around the ring, are reduced by adjusting the 240 surface-correction coils, which run azimuthally for 360 degrees along the surface of the pole faces. They are controlled through 16 programmable current elements. With adjustments made, the azimuthally averaged magnetic field in the storage volume had a uniformity of $\simeq 1$ ppm during data-taking runs.

The main temporal variation in the magnetic field uniformity is associated with radial 
TABLE VII: Selected muon storage ring parameters.

\begin{tabular}{lr}
\hline Parameter & Value \\
\hline Nominal magnetic field & $1.4513 \mathrm{~T}$ \\
Nominal current & $5200 \mathrm{~A}$ \\
Equilibrium orbit radius & $7.112 \mathrm{~m}$ \\
Muon storage region diameter & $90 \mathrm{~mm}$ \\
Magnet gap & $180 \mathrm{~mm}$ \\
Stored energy & $6 \mathrm{MJ}$ \\
\hline
\end{tabular}

field changes from seasonal and diurnal drift in the iron temperature. Because of the "C" magnet geometry, increasing (or decreasing) the outside yoke temperature can tilt the pole faces together (or apart), creating a radial gradient. The yoke steel was insulated prior to the $\mathrm{R} 98$ run with $150 \mathrm{~mm}$ of fiberglass to reduce the magnetic-field variation with external temperature changes to a negligible level.

\section{K. Relocating the Storage Ring to FNAL}

Moving the experiment to FNAL entails the disassembly and shipping of the storage ring along with all of its various subsystems. The subsystems can be disassembled and shipped conventionally, including power supplies and control systems, cryo elements and quench protection, vacuum chambers and pumping stations, electrostatic quadrupoles, shimming devices and NMR systems, and magnetic kickers. The steel plates that form the yoke, see Fig. 23, are constructed in 12 sectors each covering 30 degrees in azimuth. The plates can be unbolted and transported with a total shipping weight of 680 metric tons.

The main complication in moving the storage ring involves transporting the cryostats that contain the superconducting coils. The three cryostats, shown in cross-section in Fig. 23, are monolithic rings approximately $14 \mathrm{~m}$ in diameter. The outer cryostat holds a 48 -turn coil separated into two blocks of 24 turns to allow the muons to enter through the space between. Two inner cryostats hold 24-turn coils connected in series to the outer coil with the current flow reversed. Given the large diameter of the coils, shipping overland is not possible. Moving the coils requires airlifting them from Brookhaven to a barge off the Long 


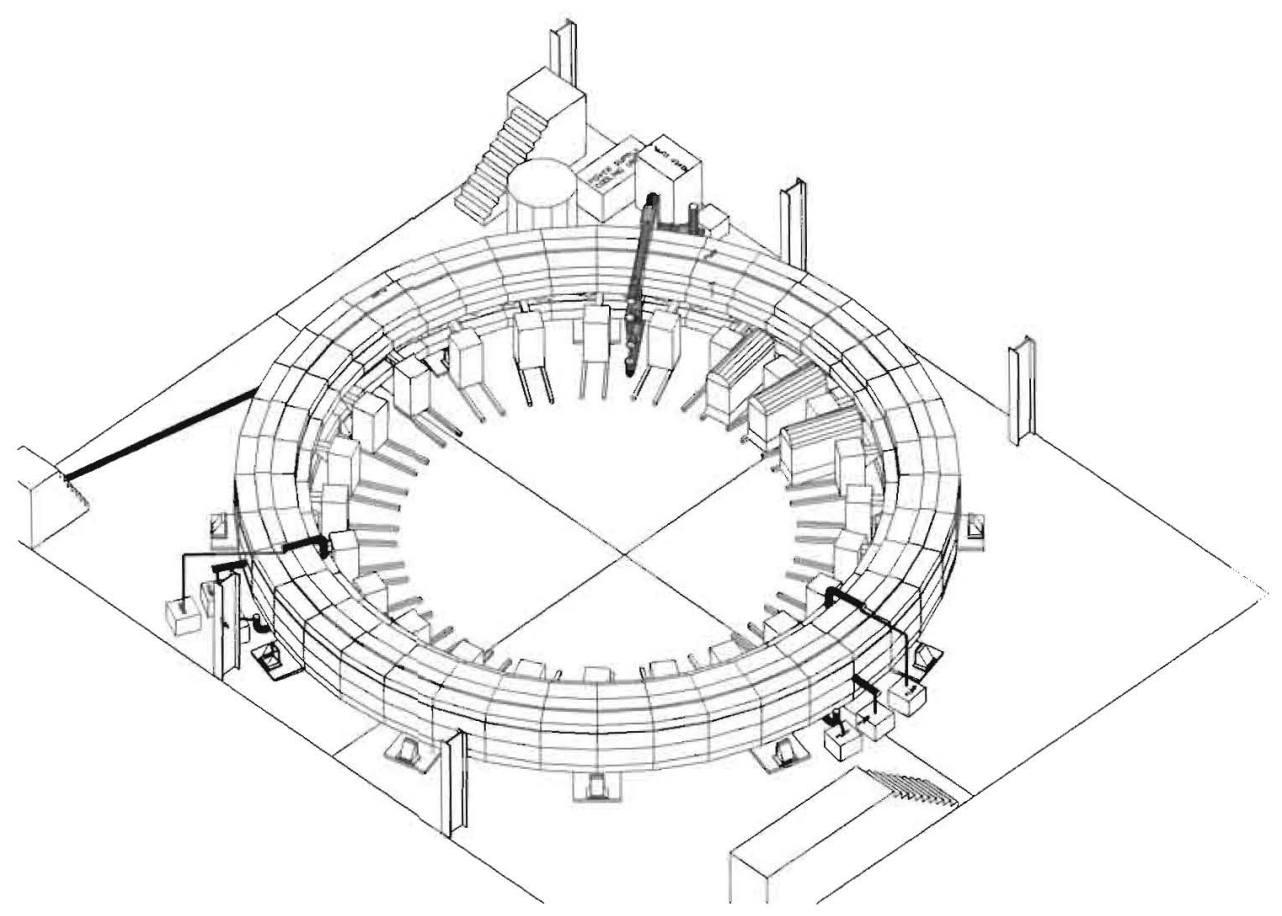

FIG. 22: A 3D engineering rendition of the E821 muon storage ring. Muons enter the back of the storage ring through a field-free channel at approximately 10 o'clock in the figure. The three kicker modulators at approximately 2 o'clock provide the short current pulse, which gives the muon bunch a transverse 10 mrad kick. The regularly spaced boxes on rails represent the electron detector systems.

Island Sound, shipping them through the St. Lawrence Seaway, and into the Great Lakes. From Lake Michigan, the barge can travel via the Calumet SAG channel to a point near Lemont, IL that minimizes the distance to Fermilab. From there, the coils can be airlifted a second time to the laboratory. The total shipping cost for the water transport is estimated at $\$ 700 \mathrm{~K}$.

One advantage of the proposed route, is the proximity of major expressways between the barge and the laboratories. On Long Island, the William Floyd Parkway, and in Illinois, I-355 to I-88, provide flight paths that avoid air space over residential or commercial areas. Erickson Air-Crane has been contacted for an initial cost estimate and consultation on feasibility. The company is an international specialist in heavy-lift applications, and their S-64 aircrane has a 25,000 lb load capacity, which is enough to transfer the heaviest of the 


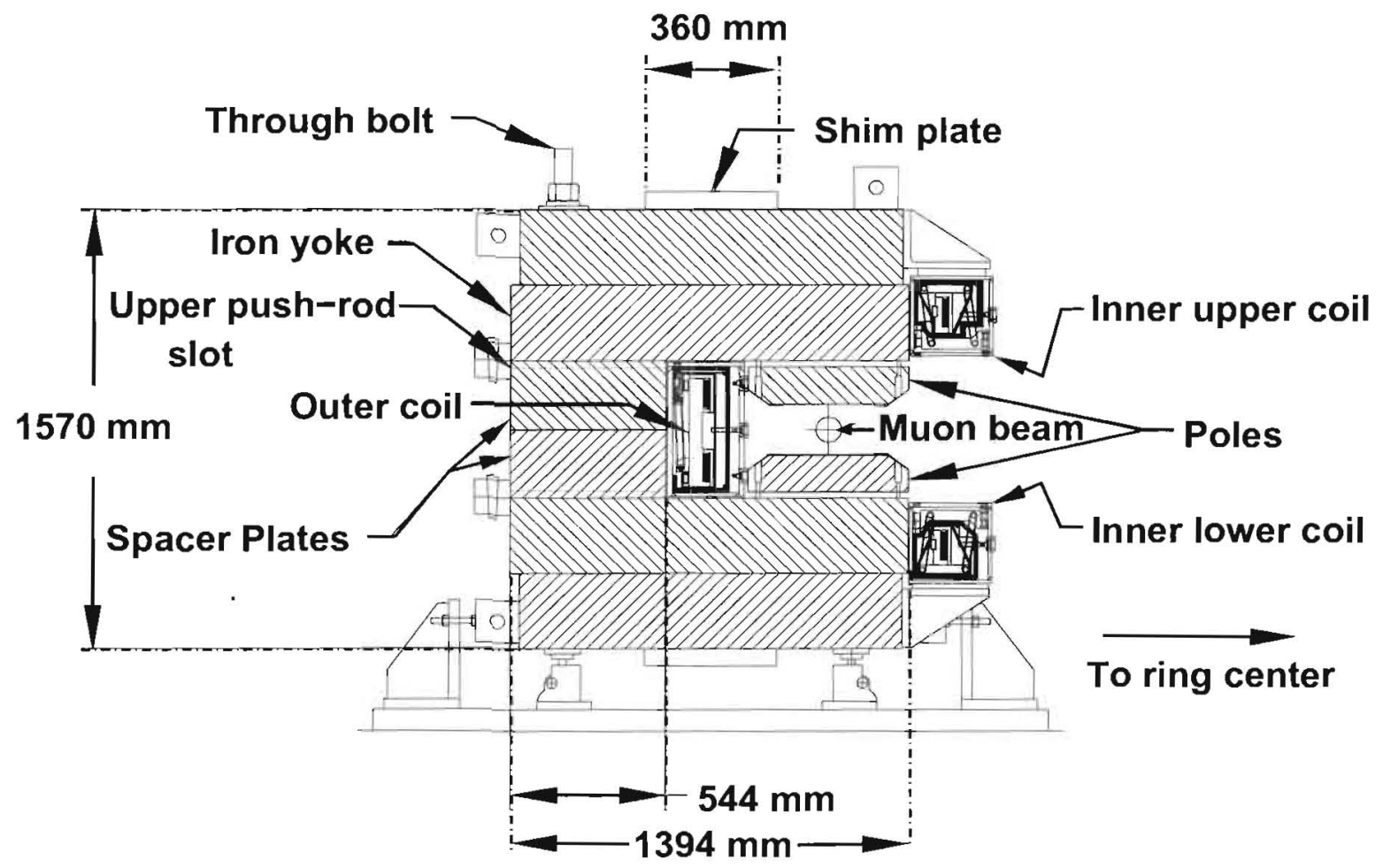

FIG. 23: Cross sectional view of the "C" magnet.

coils along with its lifting rig. The cost for the entire air crane operation at both ends of the barge voyage was quoted at $\$ 380 \mathrm{~K}$.

For the purpose of this proposal, it is assumed that the coils will be transferred from BNL to FNAL. While this provides an upper limit to the overall cost estimate of approximately $\$ 1.1 \mathrm{M}$ (without contingency) to transfer the coils, the price to re-fabricate the coils and cryostats at Fermilab needs to be explored. Given that the price to construct the cryostats and wind the coils originally totaled $\$ 2.3 \mathrm{M}$ in 1990 dollars, it is unlikely to be advantageous to consider rebuilding them. There is also a secondary advantage in utilizing a barge in that the steel from the magnet yoke can be delivered to and from the same barge for a significant savings compared to shipping overland. 


\section{THE PRECISION MAGNETIC FIELD}

We propose to measure the magnetic field in the present experiment to a precision of about $0.07 \mathrm{ppm}$ using essentially the same technique and apparatus which was used in E821. The technique was developed, implemented, and refined over a period of about twenty years. $[65-70]$. An uncertainty of $0.17 \mathrm{ppm}$ had been reached when experiment E821 was stopped (cf. table VIII).

A brief overview of the measurement is given in section VIA. Section VIB outlines the improvements that were made in the course of E821 and which resulted in the gradual reduction of the uncertainty in the field measurement by a factor of three. In section VIC we discuss the shimming procedure necessary to regain the field homogeneity after moving the magnet. In section VID we outline our plans for reestablishing the measurement after several years without operation, and how we foresee a further improvement in uncertainty to the projected $0.07 \mathrm{ppm}$.

\section{A. Methods and Techniques}

The measurement of the magnetic field in experiment E821 is based on proton NMR in water. A field trolley with 17 NMR probes was moved typically $2-3$ times per week throughout the entire muon storage region, thus measuring the field in $17 \times 6 \cdot 10^{3}$ locations along the azimuth. The trolley probes were calibrated in situ in dedicated measurements taken before, during, and after the muon data collection periods. In these calibration measurements, the field homogeneity at specific calibration locations in the storage region was optimized. The field was then measured with the NMR probes mounted in the trolley shell, as well as with a single probe plunged into the storage vacuum and positioned to measure the field values in the corresponding locations.

Drifts of the field during the calibration measurements were determined by re-measuring the field with the trolley after the measurements with the plunging probe were completed, and in addition by interpolation of the readings from nearby NMR probes in the outer top and bottom walls of the vacuum chamber. The difference of the trolley and plunging probe readings forms an inter-calibration of the trolley probes with respect to the plunging probe, and hence with respect to each other. 
The plunging probe, as well as a subset of the trolley probes, were calibrated with respect to a standard probe [74] with a $1 \mathrm{~cm}$ diameter spherical $\mathrm{H}_{2} \mathrm{O}$ sample in a similar sequence of measurements in the storage region, which was opened to air for that purpose. The standard probe is the same as the one used in the muonium measurements that determine the ratio $\lambda$ of muon to proton magnetic moments [13].

The NMR clock and the clock that measured the muon spin precession period were phaselocked to the same LORAN-C [75] signal. Systematic effects include the instrument response and were extensively studied. The leading uncertainties in the calibration procedure resulted from the residual inhomogeneity of the field at the calibration locations, and from position uncertainties in the active volumes of the NMR probes.

The ring magnet design [71], the inflector design [72], and extensive shimming contributed to the overall uniformity of the field throughout the storage ring. Figure 24 shows one of the magnetic field measurements with the center NMR probe in the trolley for E821's final data collection period in the year 2001. A uniformity of \pm 100 ppm in the center of the storage region was achieved for both field polarities and for the full azimuthal range, in particular also in the region where the inflector magnet is located.

Figure 25 shows a two-dimensional multipole expansion of the azimuthal average of the field in the muon storage region from a typical trolley measurement in 2001 . Since the average field is uniform to within 1.5 ppm over the storage aperture, the field integral encountered by the (analyzed) muons is rather insensitive to the profile and the precise location of the beam, which was determined to within a millimeter in both coordinates.

The measurements with the field trolley were used to relate the readings of about 150 (out of 370) NMR fixed probes in the outer top and bottom walls of the storage vacuum chamber to the field values in the beam region. The fixed NMR probes were read out continually. Their readings were used to interpolate the field during data collection periods, when the field trolley was parked in a garage inside of the vacuum chamber. The garage is located just outside the beam region. The uncertainty in this interpolation was estimated from redundant measurements with the field trolley within the same magnet-on period.

The field change induced by eddy currents from the pulsed kickers was measured for a prototype chamber with an optical magnetometer [73]. Time-varying stray fields from the accelerator were measured in situ with the NMR system [66, 67] and found to contribute negligible uncertainty. Another small uncertainty comes from the off-vertical field 


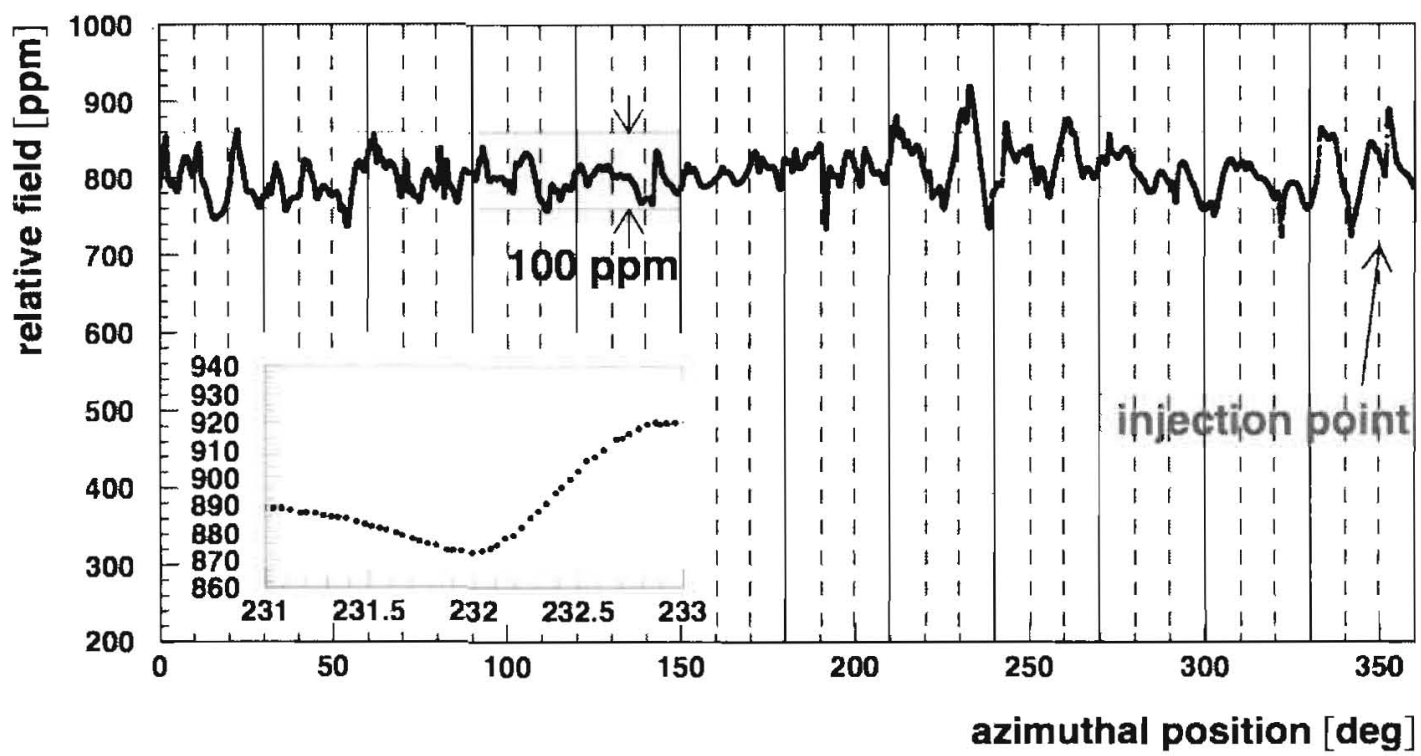

FIG. 24: The NMR frequency measured with the center trolley probe relative to a $61.74 \mathrm{MHz}$ reference versus the azimuthal position in the storage ring. These data come from one of the 22 measurements taken with the field trolley during the 2001 data collection period. The solid vertical lines mark the boundaries of the 12 yoke pieces of the storage ring. The dashed vertical lines indicate the boundaries of the pole pieces.

components [77].

The total field uncertainty is predominantly systematic, with the largest contribution coming from the calibration. For all data collection periods, the results and uncertainties were based on two largely independent analyses.

\section{B. Past improvements}

The uncertainty in the field measurement was improved by a factor of three in the course of experiment E821 and reached a final value of $0.17 \mathrm{ppm}$ for the year 2001 (cf. Table VIII).

The superconducting inflector magnet [72] was replaced between the data collection periods in 1999 and 2000 because of a damaged superconducting shield which permitted stray magnetic flux to leak into the storage region. This replacement minimized the inflector fringe field in the storage region in subsequent data collection periods and eliminated the need to measure the magnetic field with separate trolley settings in the inflector region. Together 


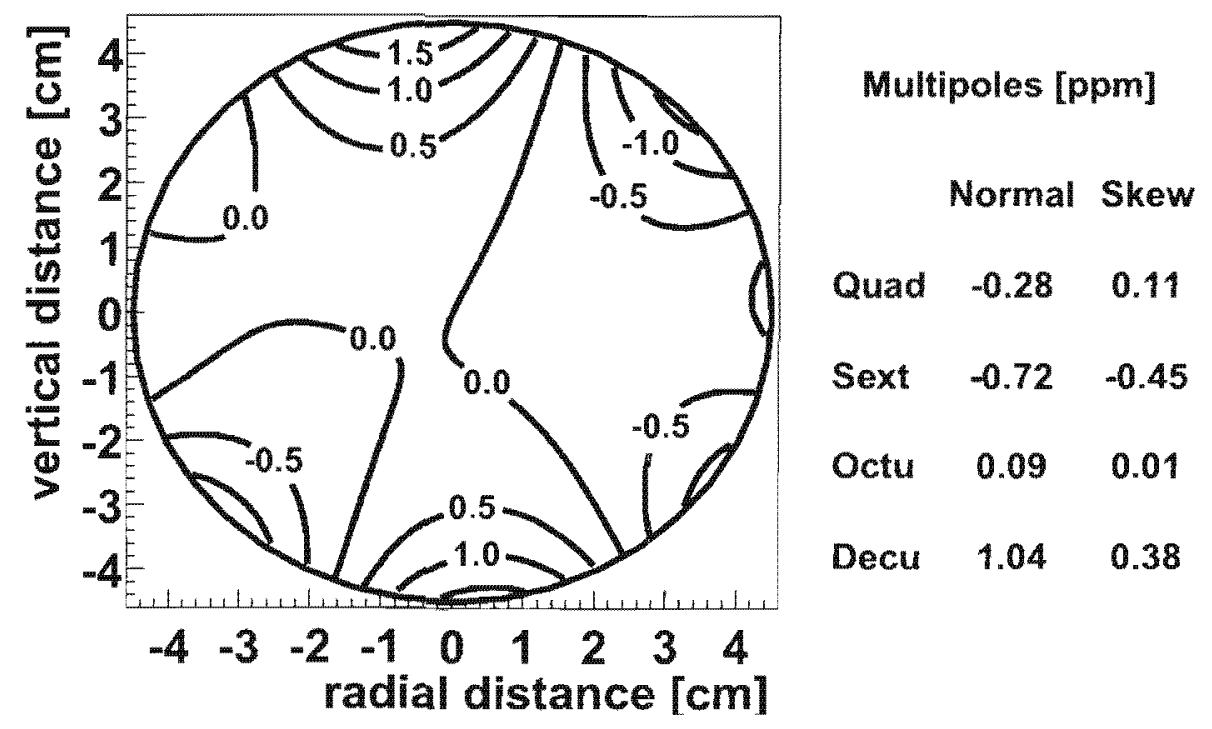

FIG. 25: A 2-dimensional multipole expansion of the azimuthal average of the field measured with trolley probes with respect to the central field value of about $1.45 \mathrm{~T}$. The multipole amplitudes are given at the aperture of the $4.5 \mathrm{~cm}$ radius storage aperture.

with refined shimming with programmable current loops, it improved the field homogeneity and thus reduced the uncertainty associated with our knowledge of the muon distribution that existed for our 1998 and 1999 results.

The addition of a plexiglass port and mirror setup to the storage ring before the 2000 data collection started, allowed us to precisely position the trolley shell at the location of a plunging probe without breaking the vacuum. It thus allowed us to make a relative calibration of the trolley probes with respect to the plunging probe during the data collection periods in 2000 and 2001, in addition to the calibrations made before and after each period.

Improvements in the alignment of the trolley rails throughout the storage ring and improvements in the trolley drive mechanism allowed us to measure the field with the trolley more often during the 2000 and 2001 data collection periods. Furthermore, we upgraded the readout of the trolley position in the storage ring before the data collection period in 2001 to reduce the uncertainty in the measurement of the average central field with the trolley.

Additional study of the trolley frequency, temperature, and voltage response resulted reduced uncertainties for the 2000 and 2001 results. 
TABLE VIII: Systematic uncertainties in the measurement of the magnetic field for experiment E821 (1998-2001) and our projections for a future effort based on known techniques and existing equipment. The uncertainty "Others" groups uncertainties caused by higher multipoles, the trolley frequency, temperature, and voltage response, eddy currents from the kickers, and time-varying stray fields.

\begin{tabular}{lccccc}
\hline Source of errors & \multicolumn{5}{c}{ Size [ppm] } \\
& 1998 & 1999 & 2000 & 2001 & future \\
\hline Absolute calibration of standard probe & 0.05 & 0.05 & 0.05 & 0.05 & 0.05 \\
Calibration of trolley probe & 0.3 & 0.20 & 0.15 & 0.09 & 0.06 \\
Trolley measurements of $B_{0}$ & 0.1 & 0.10 & 0.10 & 0.05 & 0.02 \\
Interpolation with fixed probes & 0.3 & 0.15 & 0.10 & 0.07 & 0.06 \\
Inflector fringe field & 0.2 & 0.20 & - & - & - \\
Uncertainty from muon distribution & 0.1 & 0.12 & 0.03 & 0.03 & 0.02 \\
Others & & 0.15 & 0.10 & 0.10 & 0.05 \\
\hline Total systematic error on $\omega_{p}$ & 0.5 & 0.4 & 0.24 & 0.17 & 0.11 \\
\hline
\end{tabular}

\section{Shimming the Storage Ring Magnetic Field}

The success of the experiment requires that the magnetic field be shimmed to a uniformity of $\approx 1$ ppm averaged over the storage volume. This was essentially achieved by BNL E821. A more stringent requirement of the new experiment is that the field be measured to a precision of less than about $0.1 \mathrm{ppm}$. Improvements in field uniformity over those achieved in E821 will help to reach this more challenging goal.

The magnet was assembled as a kit at BNL, and would be assembled in a similar fashion at FNAL. The magnet is made of 12 C-shaped iron yoke sectors, each in turn composed of precision engineered, low carbon steel plates. Variations in the yole plate thicknesses of the order of $200 \mu \mathrm{m}$, cause similar variations in the $20 \mathrm{~cm}$ air gap, leading to variations in the dipole field of $1000 \mathrm{ppm}$ around the storage ring. Changes in the yoke permeability from sector to sector also lead to changes in the dipole field, as do tilts, gaps, and other imperfections.

Practical mechanical tolerances thus inevitably lead to variations of the magnetic field of 
a thousand ppm. It is therefore unavoidable that reassembling the storage ring at Fermilab will lead to the loss of field homogeneity realized at Brookhaven.

Reattaining high field uniformity requires a series of shimming steps, well established by E821, from coarse to fine adjustments, and from mechanical to electrical techniques.

First the 12 upper and lower-yoke adjustment plates are shimmed by placing precision spacers between them and the yoke steel, modifying the air gap. The precision pole pieces are adjusted so that the surfaces of adjacent pole pieces are matched to $\pm 10 \mu \mathrm{m}$. The angles of the poles are measured to $\pm 50 \mu \mathrm{rad}$, and adjusted so the pole faces are horizontal when the magnet is powered.

Next, the precision pole pieces are attached to each yoke sector, separated by a $1 \mathrm{~cm}$ air gap. In the air gap are inserted a total of 864 adjustable iron wedges. The wedges were machined with an angle of $50 \mathrm{mrad}$ to compensate for the normal quadrupole moment expected from the $\mathrm{C}$-magnet design. Moving a single wedge radially by $\pm 3 \mathrm{~mm}$ changes the air gap by $\pm 150 \mu \mathrm{m}$, changing the dipole field locally by $\pm 300 \mathrm{ppm}$ (with higher order moments essentially unchanged). Finer movements allow correspondingly finer adjustments of 'the dipole field.

The air gap also contains dipole corrections coils (DCCs) which allows the dipole field over a pole to be adjusted by $\pm 200 \mathrm{ppm}$. The currents in these 72 DCCs were static in E821, but active feedback is possible.

With these tools, the variation of the dipole field around the ring can be reduced to acceptable levels.

Higher order moments of the field are reduced by shimming elements placed between the pole faces and the storage volume. These include $5 \mathrm{~cm}$ wide iron shims placed on the inner and outer radius of each pole. The edge shim thicknesses are adjusted to minimize the normal sextupole, and skew quadrupole and sextupole. It would be prudent to produce new edge shims, though it is possible none would be required.

Another tool for reducing higher order moments are the surface correction coils (SCCs). These are a set of $2 \times 120$ coils, $360^{\circ}$ in azimuth on PCBs, $2.5 \mathrm{~mm}$ apart, carrying $\pm 1 \mathrm{~A}$. The currents through the coils are set individually to reduce the average of the normal quadrupole and other moments over the ring. With these tools, a uniformity of $\approx 1 \mathrm{ppm}$ should be achievable.

It is important to mention that maintaining this homogeneity requires that the magnet 


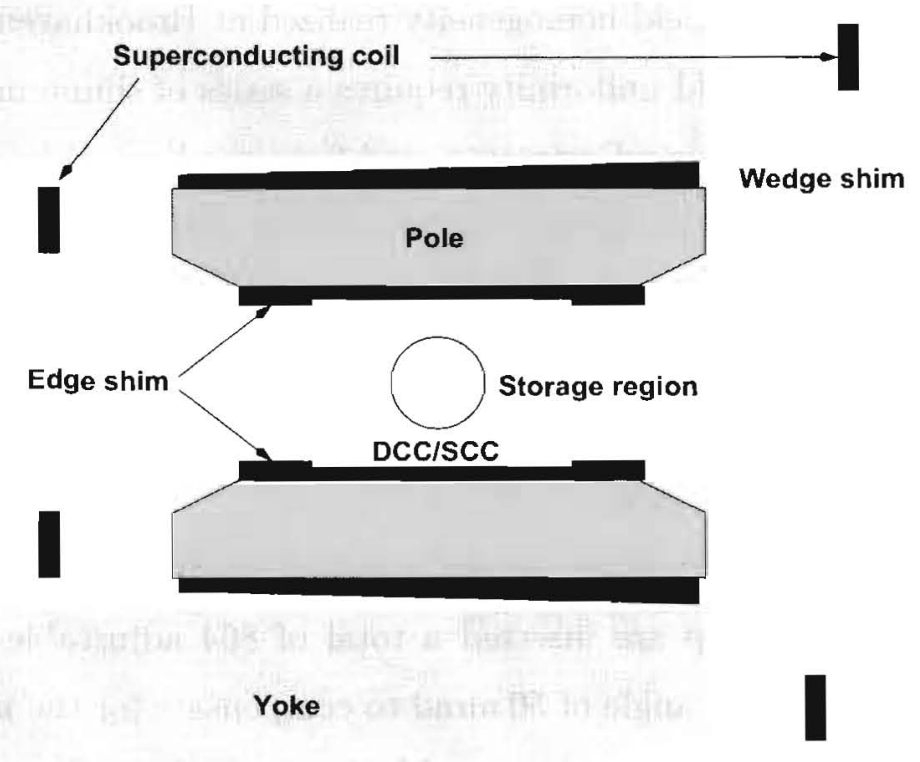

FIG. 26: Schematic view of the magnet pole region, showing the location of the various shims.

be insulated from changes in the environment. Temperature changes affect the yoke spacing, and temperature gradients in the yoke can produce quadrupole moments in the field. Insulating the magnet from temperature changes and gradients is an important part of preparing the field for the experiment.

A special shimming trolley with NMR probes will be used during the shimming process. During initial shimming the vacuum chambers will be absent. Field mapping at about $10^{5}$ points (approximately $2 \mathrm{~cm}$ apart) will be done to obtain a complete map with the trolley in a period of 4 to 8 hours. A measured set of field points can always be represented for a 2-dimensional case and the harmonic description is useful for shimming and also for analysis of errors due to field inhomogeneities. Three dimensional problems will be dealt with empirically. A computer program will provide rapid off-line analysis to represent the field in its harmonic components and with this information a decision can be made about what changes to make in the shimming configuration. In general it will be necessary to turn the storage ring magnetic field off to make changes in the iron shims.

The shimming techniques will all be done with an iterative approach involving field measurement, calculation, shimming and remeasurement. The calculation to predict the 
shimming required to improve the field will depend on the particular shimming technique being employed and on the character of the inhomogeneity.

We can anticipate at least 6 months or more will be required (once the magnet has been reassembled and powered) to shim the magnet without the vacuum chambers in place. This time would be used to adjust the pole face positions and tilts, to adjust the wedges and DCCs, make fine adjustments with the thin iron shims, and to make any changes to the edge shims. Note that some sort of mechanism for moving the shimming trolley around the ring will be necessary, as well as a means of determining its azimuthal position.

\section{Further Improvements}

The methods and techniques used in E821 are not fully exhausted; to reach a precision of $0.11 \mathrm{ppm}$, as detailed in Table VIII, only modest refinements are necessary. To reach the projected $0.07 \mathrm{ppm}$, no single approach suffices, and several systematic error sources need to be addressed simultaneously.

Our efforts to improve the existing apparatus and techniques would be focussed on the following items.

- in situ measurement of the field change from kicker eddy currents [73].

- Extensive measurements with the magnetic field trolley, aiming in particular to better resolve the position of the active NMR volumes inside the trolley shell and to map out the response functions to the level where corrections can be applied, rather than limits be set.

- More frequent measurements of the magnetic field in the storage ring during beam periods (following mechanical maintenance on the trolley drive and garage).

- Repair and retuning of a number of the fixed NMR probes to improve the sampling of the storage ring.

- Replacement of the power supplies for the surface correction coils to eliminate the occasional data loss caused by oscillating outputs.

- Refinement of the analysis techniques to reduce trolley position uncertainties in the storage ring. 
- Temperature control of the environment of the storage ring magnet.

- Additional shimming of the storage ring once the homogeneity of the E821 field has been reestablished at FNAL.

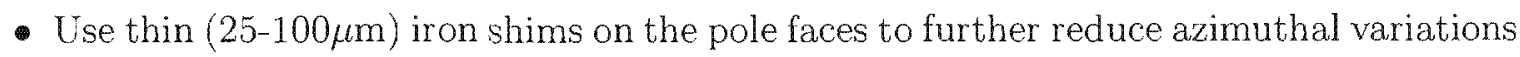
in the dipole field, which primarily couples to uncertainty in trolley position.

- Replacement of outdated computing and readout infrastructure.

- Replacement of the LORAN-C time reference by GPS-based system, if necessary.

Better knowledge of the muon beam distribution, required by the anomalous precession measurement, would also benefit the measurement of the average magnetic field.

Further improvements down to the final goal of $0.07 \mathrm{ppm}$ require significant $R \& D$. Several aspects of the measurement need to be addressed simultaneously, as no single improvement suffices to reach this level of precision. Development of new experimental techniques and equipment include

- Replacement of the water-based absolute calibration probe by a ${ }^{3} \mathrm{He}$ based system;

- Re-positioning of the fixed probes;

- Upgrade of the NMR trolley drive system;

- Upgrade of the plunging probe drive; and

- Re-machining of the precision poles.

Continued development of an independent helium-3 based standard probe [78], would benefit the field measurement, however, the projected uncertainty of $0.07 \mathrm{ppm}$ does not rely on it.

About half of the fixed probes could not be used effectively, due to their proximity to the joints in the precision pole pieces and yoke and the resulting field inhomogeneity. For those used, an empirical importance (weight factor) was assigned, depending on its location. By increasing the usable number and effectiveness of the fixed probes, the field tracking uncertainty in-between trolley runs can be further improved. An extensive simulation program, including a detailed field description that includes the effect of the magnet imperfections 
and the specifics of the fixed probes, will be needed to find optimal positions. Modification of the vacuum chambers will have to be taken into account.

Tracking of higher multipoles, and thus the interpolation uncertainty, would greatly benefit from the placement of probes in the midplane of the storage volume, rather than just above and below it. Because of the geometry of the vacuum chambers this would imply placement in vacuo and as close as possible to the horizontal quadrupole plates. At the inner radius of the ring, these probes could interfere with the placement of the calorimeters and other equipment. A probe that can operate in this environment, including a mounting and cabling scheme, has to be developed and the vacuum chambers will have to be adapted.

An increase of the speed with which the trolley moves though the ring would allow for more (frequent) measurements in the storage volume. It would simultaneously address the reduction of several sources of uncertainty, such as the trolley temperature and field interpolation. It requires the redesign of the trolley drive, together with an improvement in the alignment of the trolley rails. Besides to an increase in trolley speed, the latter will also lead to a reduction of the trolley position uncertainty, which coupled to the azimuthal field inhomogeneity affects the uncertainty in the averaged field $B_{0}$.

The relative calibration of the trolley probes can be improved by operating the plunging probe more frequently, perhaps even during each trolley run. This would require the development of a faster, more powerful plunging probe drive, which has to operate close the precision field, without affecting it at a significant level.

Finally, with the advent of more powerful magnet design tools and computer-aided machining tools, it should be investigated whether the precision poles should be re-shaped to eliminate the need and limitations of the edge shims.

The successful completion of these improvements are expected to suffice to reach the projected goal $0.07 \mathrm{ppm}$, together with the refinements mentioned before. We do note, however, that the improvement relies significantly on measurement of the field change from kicker eddy currents and the absence of field perturbations caused by the redesigned (open) inflector magnet. Neither of these have been demonstrated to a sufficient level at this time.

We are confident that a field knowledge to a precision of $0.11 \mathrm{ppm}$ can be reached using the existing experience in the field group. The present hardware has the potential to reach that level with the moderate aforementioned repairs and upgrades. A further reduction down to $0.07 \mathrm{ppm}$ appears reachable with the successful completion of a multi-facetted R\&D program 
aimed at reducing several systematic uncertainties simultaneously.

\section{VII. $\omega_{A}$ MEASUREMENT}

\section{A. Overview}

The measurement of $\omega_{a}$ is made by recording the arrival times and energies of decay positrons in a suite of 24 electromagnetic calorimeters. A position-sensitive straw chamber is placed upstream of each calorimeter to record impact positions and to provide horizontal and vertical distributions, which are important for stored beam diagnostics. Coincident events, which penetrate two or three adjacent calorimeter stations, serve as "lost muon" detectors. Additional straw chambers, placed upstream of the calorimeters, inside the vacuum, provide detailed beam dynamic information and serve as the basis for a parasitic electric dipole moment measurement. This chapter describes the systems we will use for the $\omega_{a}$ measurement, which are largely based on the experience obtained in E821.

For planning purposes, we assume that the expected rates in the new experiment will slightly exceed those of E821. The empirical argument to gauge the increase follows. The total E821 statistics of $8.5 \times 10^{9}$ events was accumulated in $2.5 \times 10^{7}$ storage ring fills. Allowing for efficiency factors consistent with those described in Table IV, E821 ran for $0.8 \times 10^{7}$ seconds using $\approx 1.5 \times 10^{20}$ protons on target (POT). In this summary of $\mathrm{E} 821$, commissioning time is excluded. At FNAL, the storage ring fill frequency will be greater by a factor of 4 and the requested data-taking period is longer by a factor of nearly 4 . Thus, the number of storage ring fills in the final sample will be greater by a factor of $\sim 15$; the 21 times increase in statistics then roughly implies that the experiment will be carried out at a rate $\sim 1.5$ times higher (we are rounding here). Under planned beam delivery scenarios, it could rise by as much as a factor of 3 . Consequently, the detectors, electronics and DAQ, will be designed to accept sustained rates per fill up to 3 times as high as BNL E821. The rate comparison is important because it guides upgraded or new systems. First, the instantaneous rate near fit start time determines the pileup fraction, which is a critical systematic uncertainty. Second, the total data flow determines the details of the electronics and DAQ systems, their data transfer rates and the total data storage required.

Higher data rates lead to the conclusion that segmented electromagnetic calorimeters 
are required to reduce the pileup fraction per channel. Additionally, the position sensitive detectors must have higher segmentation. We describe a design below, which satisfies these demands.

New waveform digitizers (WFDs) will be used to continuously digitize the analog signals from the calorimeter segments during each fill period. These data will flow to dedicated pre-frontend processors upstream of the frontend data acquisition modules where they will be packaged into event streams of derived databanks for the so-called $T$-method and $Q$ method analyses (see below). The collaboration has experience in building and running WFDs. After E821, we built more than 350 channels of $450-\mathrm{MHz}$, VME-based WFDs for several precision muon lifetime experiments at the Paul Scherrer Institute (PSI). We also built a precision clock distribution system to accurately drive all the WFDs at a known and stable frequency. The two "lifetime" experiments-MuLan [79] and MuCap [80]-- have data. rates and precision demands that prepare us for the requirements of a new $(g-2)$ effort. Together, those experiments acquired more than $140 \mathrm{~TB}$ of data, which are being processed using the NCSA computers and storage system at the University of Illinois. For the New $(g-2)$ Experiment, we will have a larger data volume, which can be stored and processed using the FNAL grid of computers and data storage. Significant computing resources also exist in the collaborating institutions for studies and analysis of the processed data. As in the past, we anticipate multiple independent approaches to the data analysis, following standard "blinding" techniques.

The traditional, or $T$ method, where individual decay-positron "events" are analyzed for time and energy, remains our primary analysis tool. Additionally, we will employ a complementary and elegant "integrating" method, the $Q$ method. The $Q$ method amounts to digitizing the energy deposited in an entire calorimeter (all segments) vs. time following injection. No threshold is necessary; all samples are recorded without bias and summed. The method is robust and intrinsically immune to pileup, but it is new and other systematics will likely emerge.

In the $T$ method, positron decays are recorded individually and are sorted by energy and time. For each positron recorded at time $t$ and having energy greater than $E_{t h}$, a single count is incremented in a histogram, such as the one shown in Fig. 3 . The asymmetry is determined by the choice of threshold, and the statistical power is proportional to $N A^{2}$. Optimizing this figure-of-merit implies setting $E_{t h}$ between 1.8 and $1.9 \mathrm{GeV}$. The $T$ method is well 
understood by the collaboration; we use it to determine event rates and running necessary for the goals of this proposal. A slight variant on the $T$ method is an asymmetry-weighted (or energy-weighted) event mode, a $T^{\prime}$ method. Here, individual events having energy $E_{i}$ are weighted by their asymmetry, $A_{i}$. This method improves the statistical power of the $T$ method by about 10 percent at a cost of modestly increased demands on the gain stability of the detectors. The $T^{\prime}$ method can be derived from the standard data set and requires no additional hardware or special data-taking procedures. Variants of this method were used as part of the analysis approaches applied to the 2000 and 2001 data-taking periods in the E821 experiment.

In contrast, the $Q$ method does not rely on the separate identification or isolation of positron events. It involves integrating the energy deposited in the entire calorimeter, plotted as the summed energy vs. time. In this simple method, the energy deposited, which is proportional to the light in the calorimeter, is digitized for the entire fill and the digitized samples are in turn added from fill to fill to produce a final histogram. The histogram can be fit by the same function used to fit the $T$ method data. The asymmetry is lower compared to the $T$ method because all accepted events are used, even the small fraction of low-energy positrons that hit the calorimeter and carry negative asymmetry compared to the higher-energy positrons (See Fig. 2b). The discrete placement of the detectors ensures a higher comparative acceptance of the highest energy positrons, and a net asymmetry approximately half that of the $T$ method. In the $Q$ method, a greater number of events are included, thus the effective $N$ is larger. We have performed a GEANT simulation to compare the $T$ and $Q$ methods. The simulation is based on tracked muons through the storage ring and features details such as the coherent betatron oscillation, which modulates the detector acceptance. We ignored that small effect in fitting the data; the result is a poor $\chi^{2} /$ dof, but it otherwise does not affect the comparison. Figure 27 shows spectra prepared using the $T$ and $Q$ methods, both fit with the five-parameter function:

$$
N \exp (-t / \gamma \tau)[1+A \cos (\omega t+\phi)]
$$

In the upper panel, the number of events having positron energy greater than $E_{t h}=1.8 \mathrm{GeV}$ is plotted vs. time after injection. The fit gives an uncertainty on $\omega_{a}$ of $59 \mathrm{ppm}$ for this sample. The bottom panel shows the same simulation, but the plot represents calorimeter energy vs. time after injection. The uncertainty on $\omega_{a}$ is $65 \mathrm{ppm}$; the $Q$ method is statis- 

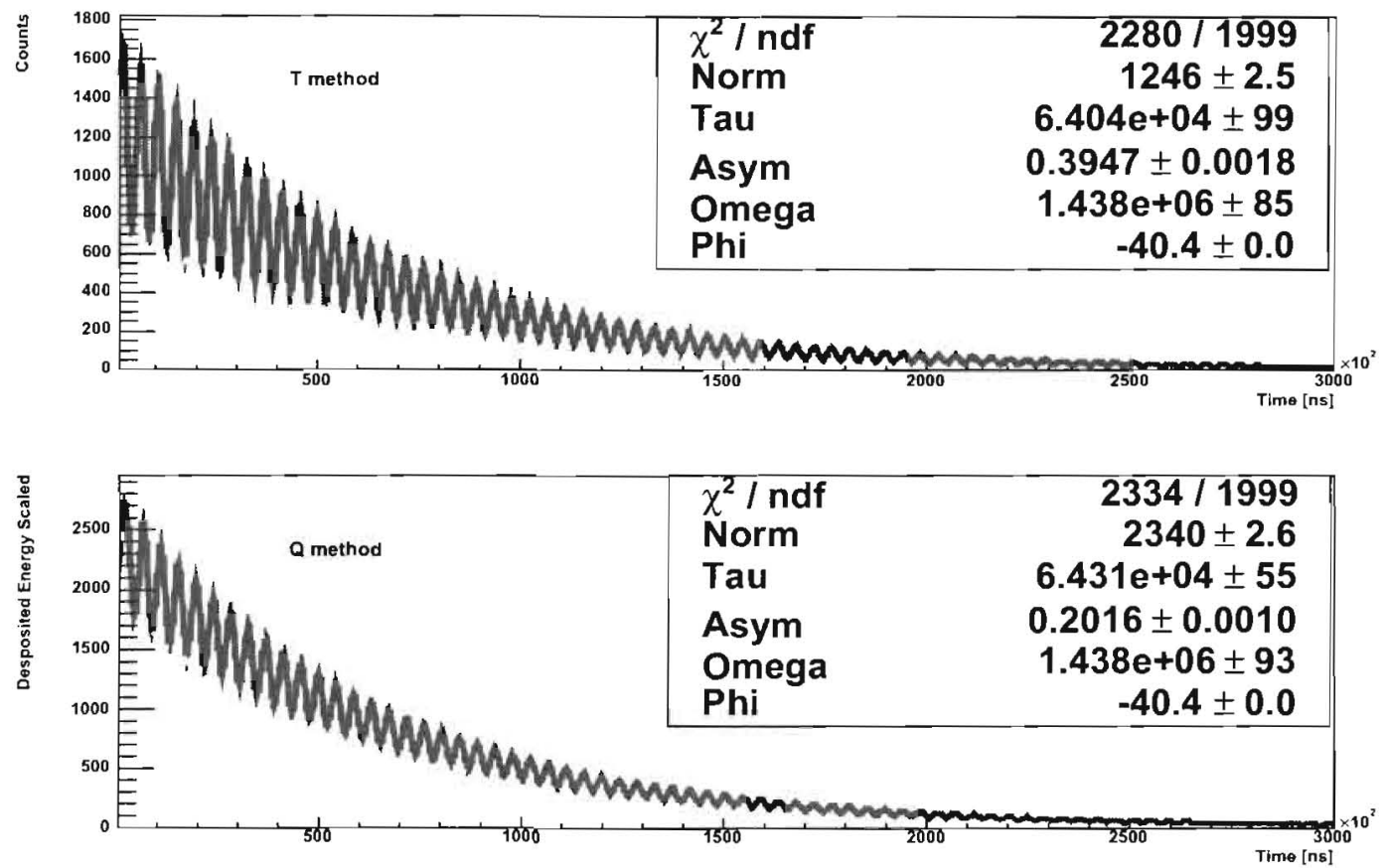

FIG. 27: GEANT simulation of events. Upper panel: Data analyzed using the tradition $T$ method with $E_{t h}=1.8 \mathrm{GeV}$. Lower panel: Data prepared using $Q$ method, representing energy vs. time. Note the poor $\chi^{2} /$ dof for each plot is because the fits were performed using a simple 5-parameter function, which ignores the coherent betatron oscillations present in the simulation.

tically weaker than the $T$ method by about 9 percent, implying an 18 percent longer run is necessary to obtain the same precision. However, the $Q$ method has an interesting advantage. There is no pileup correction to be made so the increased rate will not complicate the analysis algorithm. While the $Q$ method had been recognized as viable during the E 821 effort, it was impossible to implement with the existing WFD hardware and unattractive to use because of the significant hadronic flash, which added a large and slowly decaying baseline for many of the detectors in the first half of the ring. Our new digitizers will be capable of storing all the samples from a complete fill so $Q$-method running can be enabled as a parallel data stream; the anticipated smaller hadronic flash should keep the pedestal baseline relatively flat. 


\section{B. Electromagnetic Calorimeters}

The electromagnetic calorimeters for the E821 experiment used a Pb/SciFi design [81, 82], Each calorimeter consisted of a monolithic block of 1-mm diameter fibers arranged in a near close-packed geometry within grooved lead alloy foils. The fractional composition of the detector was $\mathrm{Pb}: \mathrm{Sb}:$ Fiber:Glue $=0.466: 0.049: 0.369: 0.096$ (by volume), leading to a radiation length $X_{0}=1.14 \mathrm{~cm}$. The fibers were oriented radially so that the positrons would impact on the detector at large angles with respect to the fiber axis. Four lightguides directed the light to independent PMTs and the summed analog signal was processed by waveform digitizers. The $14-\mathrm{cm}$ high by $22.5-\mathrm{cm}$ radial by $15-\mathrm{cm}$ deep calorimeter dimensions were largely dictated by the avalable space and the need to have a sufficient radial extension to intercept the positrons. The energy resolution requirement for $(g-2)$ is relatively modest, $\sim 10 \%$ or better at $2 \mathrm{GeV}$.

For the New $(g-2)$ Experiment, the systematic errors associated with gain instability $(0.12 \mathrm{ppm})$ and pileup $(0.08 \mathrm{ppm})$ must each be reduced by a factor of $\sim 3-4$. We have designed [15] a new calorimeter that retains the fast response time of plastic scintillating fiber, but is made from an array of dense submodules where each is oriented roughly tangential to the muon orbit. This configuration provides transverse segmentation and allows for multiple simultaneous shower identification. A 50.50 ratio of tungsten to scintillator (and epoxy) reduces shower transverse and longitudinal dimensions. The calculated [84] radiation length, $X_{0}=0.69 \mathrm{~cm}$, is $60 \%$ of the length for the $\mathrm{Pb} / \mathrm{SciFi}$ madules used in E 821 . Consequently, the modules can be made compact enough to free space for downstream readout in the highly constricted environment of the storage ring. The high density leads to a smaller radial shower size, which improves the isolation of simultaneous events. We find that using $0.5-\mathrm{mm}$ layers gives an acceptable resolution close to $10 \%$ at $2 \mathrm{GeV}$ for a prototype we have built and tested; a non-trivial error contribution to this measured performance parameter came from the beam momentum spread, photo-electron yield and transverse leakage fluctuations in our test environment. Therefore, the intrinsic detector response from sampling fluctuations alone is better.

A plan view of the vacuum chamber and the detector positioning is shown in Fig. 28, indicating that this design looks promising for the standard vacuum chamber sections. In this figure, 20 lightguides are indicated as they curl toward the inside of the storage ring. By 


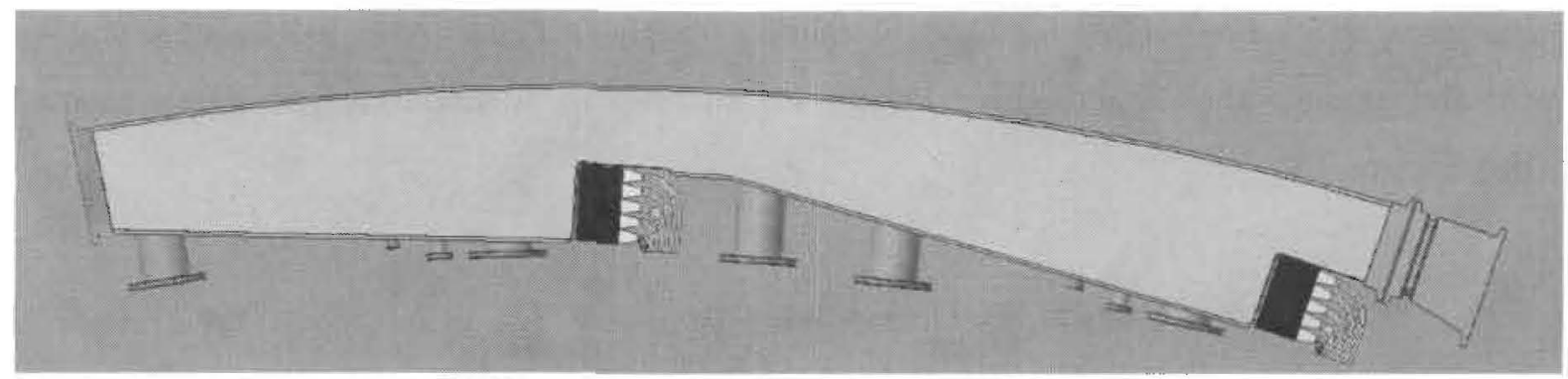

FIG. 28: Plan view of new calorimeters and existing scalloped vacuum chamber region.

design, the $\mathrm{W} / \mathrm{SciFi}$ is a single monolithic array, which can be readout on the downstream side by any segmentation of optical couplers. The choice of 20 or 35 readouts $(4 \times 5$ array or $5 \times 7$ array) is an optimization to be determined based on the final readout solution. We are exploring silicon photomultiplier (SiPM) arrays and will perform tests in the coming year. At the time of this proposal, several large SiPM arrays are being produced, which would nicely match the 35-segmented model mentioned above. However, a conservative solution will be to use PMTs located outside of the field region. It is a solution that we have considerable experience in implementing based on E821.

Appendix $\mathrm{C}$ includes a more detailed description of a tungsten / scintillating fiber (WSciFi) sampling calorimeter that meets these demands. In anticipation of this proposal, we built a $4 \times 6 \mathrm{~cm}^{2}$ prototype module made of $0.5-\mathrm{mm}$ pitch layers of fiber ribbons and pure tungsten plates. Measurements were made at PSI and at FNAL and results have been reported [15].

\section{Position-Sensitive Detectors}

In E821, five-fold, vertically segmented scintillator hodoscopes were mounted on the upstream side of each calorimeter. To provide impact position information for shower reconstruction and to obtain a better horizontal and vertical profile, we propose to use a system of straw detectors in front of each station. These can be relatively simple detector systems with standard multi-hit TDC digitized readout. The time-start for the straws will be derived from a summed signal from the calorimeters. The straw system in front of each calorimeter will provide information for shower impact, pileup identification, and muon loss monitoring. For some stations, a complement of in-vacuum straws will serve as positron traceback 
detectors, which are needed for beam dynamics imaging. These extended imaging stations will also provide the data used in the physics analysis for a muon electric dipole moment (See Section IX).

\section{Waveform Digitizers for Calorimeter Readout}

The primary data acquisition challenge for a new experiment is the readout and interpretation of the calorimeter signals. For the New $(g-2)$ Experiment, we intend to gather this information via waveform digitization, where the PMT analog output waveforms are continuously digitized at high speed. While waveform digitization was used to great effect in E821, significant advances in many fields - ranging from high speed analog circuitry to parallel computation - will allow us to both dramatically simplify and miniaturize the hardware while extracting significantly more information from the raw data.

The E821 $400 \mathrm{MHz}$ waveform digitizer (WFD) hardware was based on an earlier design from the MACRO experiment. It consisted of an analog input shaper, a clock input module, the Flash digitizer itself, a data formatter, discrete RAM banks, and a VME32 interface. The relatively low RAM densities available at the time coupled with stringent realtime processing constraints required the time consuming design and high-cost implementation of a custom data formatting ASIC. Current off-the-shelf and custom WFD designs are generally similar, but typically contain on-board FIFO memories. The main difference lies in the replacement of the inflexible custom ASICs with field programmable logic (in the form of FPGAs or CPLDs). We have deployed such a design for use in the MuLan and MuCap precision muon lifetime experiments at PSI.

For the New $(g-2)$ Experiment, we propose a departure from this model. FPGA based designs excel at manipulating very low level, realtime logic transformations, including such things as ADC readout, memory controllers, and network transceivers. High level data manipulation on the devices, however, is complicated by relatively high implementation, testing, and debugging barriers. These manipulations are much better done in high level computer programming languages on commodity hardware. We propose a hybrid design that merges the strengths of each approach, while minimizing engineering; construction, and deployment costs.

The time structure of PMT pulses requires digitizing the output data stream at $500 \mathrm{MHz}$ 


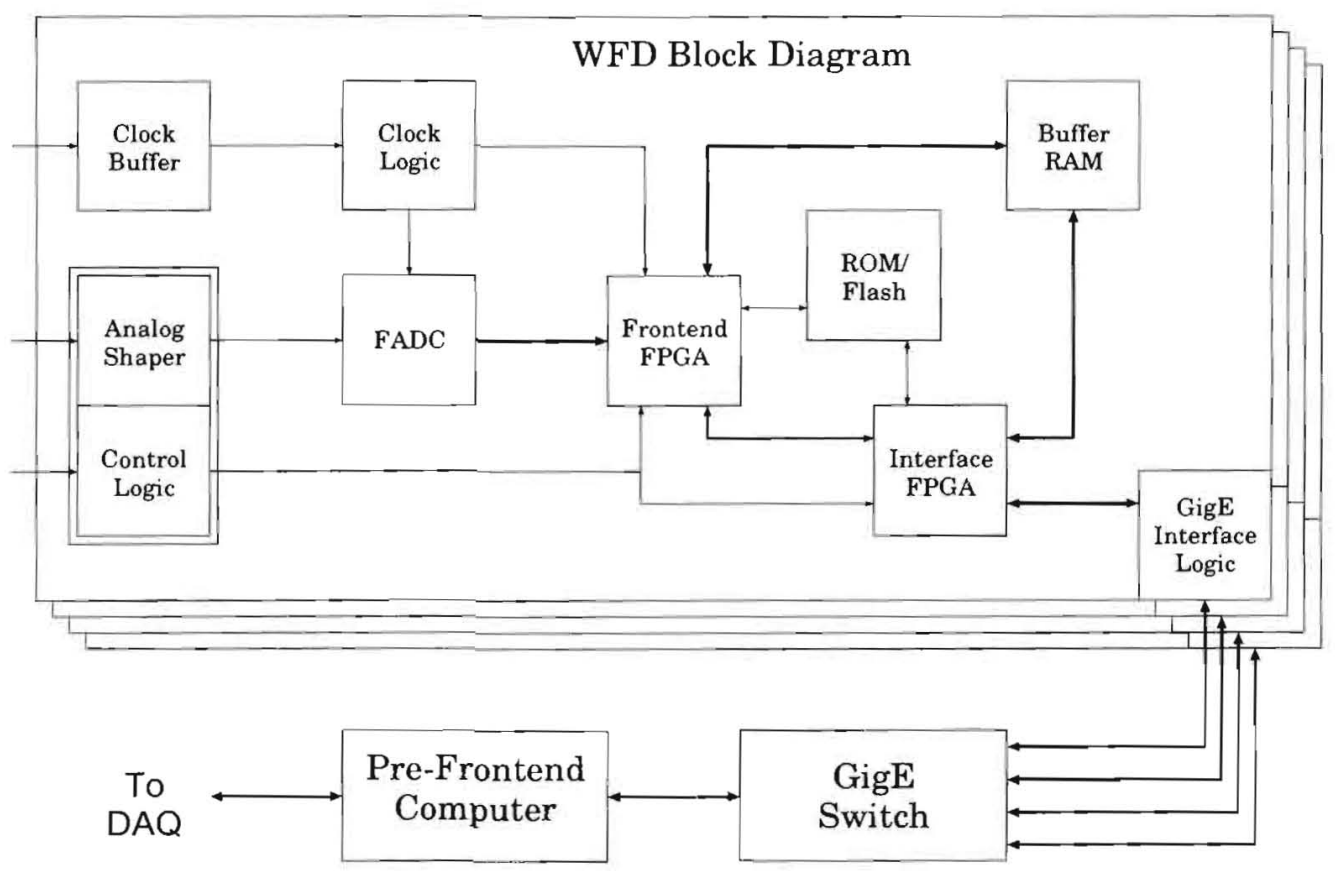

FIG. 29: A block diagram of the hybrid waveform digitization system.

with an 8 bit flash digitizer. We will continuously digitize these signals, recording $600 \mu \mathrm{s}$ of data per calorimeter segment per fill. Having every sample of each fill permits simultaneous extraction of $T$-method, $Q$-method, and other derived data streams from one digitization record. Ideally, we would simply send all of this raw data to persistent storage. With 24 calorimeters, however, the total raw data rate is $3-5 \mathrm{~GB} / \mathrm{s}$ (depending on segmentation), significantly too great a rate to store completely. The DAQ system needs to reduce this, to of order $50-100 \mathrm{MB} / \mathrm{s}$; the final value will of course depend on details of the DAQ system and the available FNAL network infrastructure and data storage resources at the time the experiment is run. To meet this challenge, the hybrid digitization system will operate in two stages: a simple hardware digitizer to record the data, and "pre-frontend" computers to perform all triggering, data selection, and packaging tasks. The hybrid system will present a configurable set of packaged data streams (e.g. T/Q-method datastreams) to the DAQ system for collation and storage.

Each WFD hardware channel will consist of analog and clock input stages, a $500 \mathrm{MHz}$ flash digitizer, buffer memories, the communication interface, and various support modules (firmware PROMS, programming and test ports, LED feedback, synchronization hardware, etc.). These will be tied together with one or more FPGAs to move the data at high 
speed between the various functional blocks (see Figure 29). Minimizing the duration of the design life cycle is of great importance, and we approach this in part by using off-the-shelf technologies whenever possible:

- We intend to use standard DDR computer memories in a bank switched configuration, instead of more expense FIFO memories. This allows simultaneous storage of the current fill while reading out the previous fill. It also permits use of previously written and debugged memory controller firmware.

- Every channel will operate independently (although multiple channels may be carried on one physical circuit board, they should share no processing or interface resources); this simplifies the firmware for setup and communications, as well as simplifying replacement of failed channels.

- The boards will be mounted in VME-style crates to supply the large quantities of power needed, but the communications interface will be Gigabit (or faster) ethernet. Again, debugged firmware modules are readily available, and custom interconnect topologies can be implemented with inexpensive commercial hardware. We may even consider UDP or TCP transport instead of using raw ethernet communications.

- The raw data can be compressed before transport if necessary. We will utilize a standard algorithm (such as the LZ77 algorithm embodied in the ubiquitous gzip library) to minimize implementation and debugging costs.

As each WFD channel is independent, while positrons will typically deposit energy in multiple calorimeter segments, triggering decisions must be made globally over each calorimeter. In the past, we might have performed this task in a separate analog module which would then force digitization in each WFD channel. Recent advances in multicore and parallel computation (embodied at the consumer level, for instance, in the popular Intel Core2 CPU architecture) and practical parallel programming techniques will allow us to perform this task cost effectively with off-the-shelf hardware and software written in a high level language. For our needs, General Purpose Graphics Processing Units (GPGPUs), consisting of multiple high-speed Hoating point units with hundreds of cores per die, are becoming readily available at low cost. The available programming interfaces, such as NVIDIA's CUDA, 
AMD's Stream, and the developing OpenCL standard hide the complexity of data transfer and core scheduling, permitting relatively simple access to these massively parallel resources.

Each calorimeter station, then, will have a dedicated "pre-frontend" computer that will perform WFD readout and triggering for a given calorimeter. This pre-frontend will decompress and reformat the WFD data to optimize computation speed. It will then perform a number of parallel computations over each calorimeter:

- For the T-method, equal-time samples must be summed over all calorimeter segments, triggers identified, and "data islands" formatted and written to a data stream.

- For the Q-method, blocks of consecutive samples must be decimated and summed across calorimeter segments. Successive fills can being summed together to further reduce the data rates.

- A potential new approach to pileup correction sums multiple successive fills before performing the $T$-method triggering decisions. This additional "Pileup $T$-method" stream could be easily derived from multiple recorded streams, and saved along with the standard $T$-method data set.

- Occasionally, entire fill records should be stored for detailed studies of, for instance, gain and pedestal stability.

Each of these potential computations, and others that are identified in the future, can be independently formatted, packaged and presented to the DAQ system for persistent storage. By performing all of this high level physics in software we can defer the actual specification of the final data streams until very late in the experiment, once physics studies and data acquisition performance tests have been performed.

\section{E. Clock systems}

A time base having $0.01 \mathrm{ppm}$ absolute accuracy and stability over several months is not difficult to obtain; vendors such as Precision Test Systems and Agilent provide inexpensive synthesizers driven by ovenized oscillators which meet that specification. In the MuLan experiment, for instance, the $500 \mathrm{MHz}$ system clock which drove the WFDs was generated with an Agilent E4400 synthesizer. Extensive comparisons with external standard oscillators, 
both before, during, and after the experiment, confirmed the stability and accuracy claims of the manufacturer. The long term stability of such devices can be economically extended to cover the multiyear duration of this experiment by slaving the master synthesizers to a common GPS-disciplined $10 \mathrm{MHz}$ frequency standard.

Similar synthesizers will be used in the present experiment to drive the various acquisition and NMR systems. We have extensive experience in distributing similar clock signals in other precision experiments, using low skew linear fanout and amplifier modules from companies such as Miniciruits. The final component of the clock system design is a robust blinding methodology; we hide the absolute clock frequencies from the $\omega_{a}$ and $\omega_{p}$ analysis teams until the analysis is completed. These are well understood techniques with very low design and implementation risks.

\section{F. Data Acquisition}

By comparison to E821 the New $(g-2)$ Experiment will record about $21 \times$ the decay electrons at $\sim 1.5 x$ the rate per fill and $\sim 6 \times$ the rate per second. Moreover, the experiment will record the signals from the individual calorimeter segments rather than the calorimeter-segment sums, and record $T$-method datasets (comprising digitized pulses islands), Q-method datasets (comprising digitized fill periods) and other derived datasets. In addition, the new readout must incorporate the new straw counter arrays and account for the different beam time structure.

The new data acquisition must handle both very high data rates $(\sim 80 \mathrm{MB} / \mathrm{sec})$ and very large raw data volumes ( $\sim 1$ PB total). It must transfer both the event data from various detector sub-systems to the mass storage devices and the experimental parameters from the various diagnostic sub-systems to an experiment database. Moreover, the readout system must be deadtime-free during the measuring periods in order to avoid any distortions of the time spectrum of the decay positrons. Finally, the DAQ must be flexible enough to use for the installation, testing, diagnostic and production phases of the experiment.

The DAQ (see Fig. 30) will be implemented as a modular, distributed acquisition system on a parallel, layered processor array using multi-threaded PC's, a Linux platform and a multi-layered Gbit network. A frontend (FE) layer will be responsible for the readout of the calorimeter segment waveforms, straw counter hits and other detector sub-systems. The 


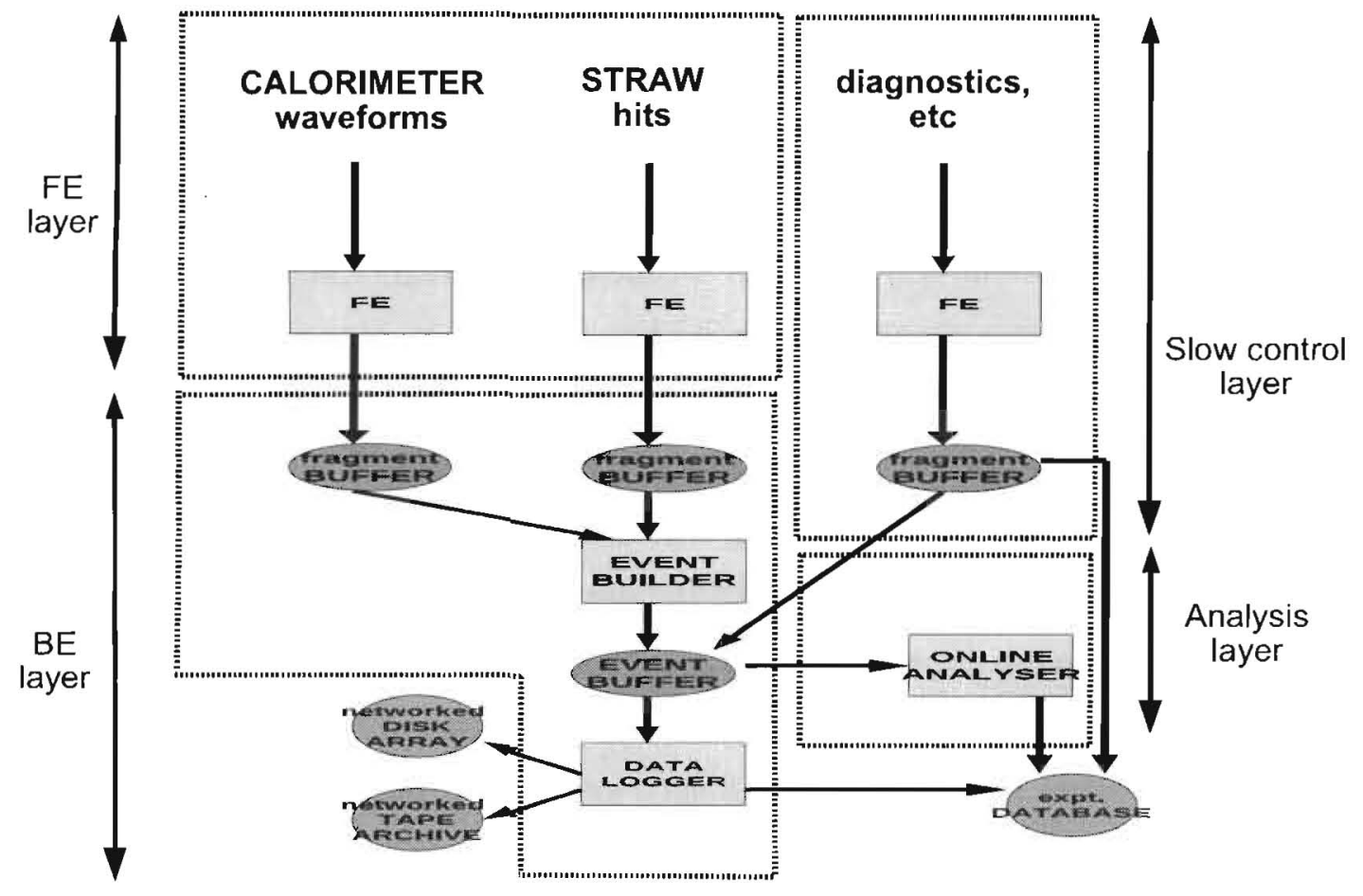

FIG. 30: Schematic layout of the anticipated DAQ system.

backend (BE) layer will be responsible for both the assembly of data fragments into complete events and the permanent storage of the complete events. A slow control layer will be responsible for the control and monitoring of diagnostic instrumentation associated with the ring, detectors and other sub-systems. Finally, an online analysis layer will be responsible for the integrity-checking and basic histogramming that ensures the overall quality of recorded data.

The primary source of high-rate data is the twenty-four calorimeters. As described earlier, each calorimeter segment is instrumented with one waveform digitizer channel that transmits packets of $500 \mathrm{MHz}, 8$-bit, continuous digitization (CD) data to so-called pre-FE processors. These pre-FE processors derive the $Q / T$-method data-streams and transmit the resulting derived databanks over the FE network to the FE layer of the data acquisition. We expect a total rate of calorimeter $Q / T$-method data of roughly $80 \mathrm{MB} / \mathrm{sec}$. 
A second source of high-rate data are the straw counter arrays. We envisage a straw counter readout system based on commercial VME multi-hit TDCs and VME-to-PCI interfaces that deliver their data to a dedicated FE processor on the FE network. We expect a total rate of straw counter data of several $\mathrm{MB} / \mathrm{sec}$.

The backend layer will receive data fragments from the various frontend processors across the frontend network, assemble the event fragments into complete events, and copy the events to mass storage. Each event will represent a time-ordered history of the calorimeter, straw counter, and other detector data for one entire fill. For mass storage we propose to store one copy of the data on the Fermilab tape archive (i.e. the Fermilab Enstore system or its future equivalent) and one copy of the data on a large disk array (e.g. a BlueArc storage node or its future equivalent). The event building will utilize backend local memory segments for temporary buffering of event fragments and the data logging will utilize backend local disks for temporary buffering of complete events.

The slow control layer is essential for careful monitoring of systematic issues and will incorporate the readout of instrumentation such as HV controls, current monitors, temperature sensors, field probes and scalars. The slow control readout will operate in periodic mode and be asynchronous to the beam cycles, DAQ cycles, etc. The slow control data will be written to both the mass storage devices and the experimental database.

The online analysis layer will provide the integrity checks and diagnostics plots that ensure the quality of recorded data. The online analysis system will be resident on a dedicated network and receive events as 'available' from the backend layer (in order to avoid the introduction of unnecessary deadtime). The system will provide for both local and remote access to the experimental data.

\section{SYSTEMATIC UNCERTAINTIES ON $\omega_{A}$}

In this section we consider the primary systematic errors on the $\omega_{c}$ analysis, which totaled $0.19 \mathrm{ppm}$ in the final run of E821. [104] Our goal in the New $(g-2)$ Experiment is a factor of 3 reduction for a total of $\leq 0.07 \mathrm{ppm}$. This goal can be met by incorporating a suite of improvements to the experiment. Here, we briefly outline the plan to reduce the largest sources of systematic error: gain changes, lost muons, pileup, coherent betatron oscillations, and the uncertainty associated with the electric field and pitch corrections. Table IX lists the 
final E821 uncertainties and projections for improvements in the New $(g-2)$ Experiment. The traditional $T$ method analysis is assumed because uncertainties can be reliably projected based on our considerable experience in these analysis efforts. Since the $Q$ method is new, we have not included its positive and partially independent impact on the final statistical result, nor are we able to fully project associated systematics. One key attractive feature of the $Q$ method is pileup immunity; there is no correction necessary so that systematic uncertainty is absent. Comparing the analysis results using both $T$ and $Q$ methods will provide a valuable confirmation that systematic errors are understood.

\section{A. Gain Changes and Energy-Scale Stability}

The hardware gains of the detectors were determined to be stable to $\approx 0.15 \%$ from early to late times within a storage ring fill. This limit was established by plotting the average energy for each $(g-2)$ period versus time after the PMTs were switched on. The gating circuitry in the base that allowed the PMTs to be turned off to avoid the initial burst of pions entering the ring, also resulted in a variation in the gain. For gain variations like this one, where the time constant is long compared to the $(g-2)$ oscillation period, the coupling to the $\omega_{a}$ frequency is small and after correction the residual systematic error is less than $0.02 \mathrm{ppm}$.

If the gain oscillates at a frequency $\omega_{a}$, with an amplitude that varies in time, and with a phase that differs from that of the $\omega_{a}$ oscillation of the positron sample, then a direct error on the measured value of $\omega_{a}$ is produced. The average rate at which energy is deposited into the calorimeters oscillates with frequency $\omega_{a}$, and therefore any rate dependence in the gain of the detectors produces gain oscillations. We were able to demonstrate that the gain dependence on rate was small enough that its effect on $\omega_{a}$ was typically less than $0.03 \mathrm{ppm}$. In the new experiment, the slightly increased beam rates will be offset by increased detector segmentation. In E821, a UV-laser system was used to periodically pulse the scintillator in the detectors and thus monitor the complete gain and reconstruction chain during data collection against an out-of-beam reference counter. Unfortunately, the light distribution system included too many branches and only one upstream reference detector. Small fluctuations cascaded so that gain stability could be monitored to no better than a. few tenths of a percent. At PSI, we have recently built and used a simplified version of 
this system, which monitors hardware gains at the sub- $0.1 \%$ level by having a monitor on a parallel level to that seen by the detectors. We intend to incorporate a similar system in the New $(g-2)$ Experiment to largely eliminate this uncertainty.

The greater contribution to the gain systematic error came from artificial gain oscillations at the $\omega_{a}$ frequency, introduced by the data reconstruction software. Here, the "energyscale" stability is related to software reconstruction of waveforms. When a signal was above the WFD hardware threshold, a pre-set minimum number of sequential WFD samples was recorded. These data were fit offline for the peak plus linear background to deduce the energy and time of the positron. But, if the trigger pulse was followed or preceded closely by another pulse, both pulses were fit together with a common background term, and the fitting region becomes longer compared to that used for a single pulse. The fitted energy was found to depend slightly on the length of the fitting region and the fitting samples were fixed in number by hardware. Because the data rate oscillates at frequency $\omega_{a}$, and is higher at early than at late decay times, it follows that the fitting region length oscillates at $\omega_{a}$ and is, on average, longer at early times compared to late times. This produces a small, effective gain oscillation with frequency $\omega_{a}$ whose amplitude decreases with time, leading to a systematic error on $\omega_{a}$. Given the current capabilities in data throughput, the new electronics will record all samples in place of isolated islands, thus removing the source

of this reconstruction bias. In summary, the larger of the gain systematic pieces will be eliminated by design and the smaller contribution will be monitored more precisely.

\section{B. Lost Muons}

"Lost muons" refers to muons that escape the storage ring before they decay. These losses were about $1 \%$ per lifetime at early decay times and decrease to about $0.1 \%$ at later decay times in the BNL experiment. One consequence of losses is that, in a fit to the data, the lifetime is not quite correct. This is a slow change in the spectrum, having no $\omega_{a}$ frequency component; therefore the correlation to $\omega_{a}$ in the fit is small. However, even though the correlation is small, neglecting muon losses in the fit in E821 would have shifted the $\omega_{a}$ frequency by $0.18 \mathrm{ppm}$ and resulted in a very poor $\chi^{2}$ from the fit. By monitoring the muon losses with hodoscopes on the front of 14 of the calorimeters in E821, the muon loss profile was constructed and the resulting uncertainty was held to better than $0.03 \mathrm{ppm}$. In the 
upgraded experiment, all 24 calorimeters will incorporate muon sensitive detectors, straws or scintillator, allowing muon losses to be monitored around the entire ring. Furthermore, an open-ended inflector will reducing scattering as the muons enter the storage ring, resulting in smaller losses. Finally, muon losses can be greatly reduced when an effective "scraping" strategy is employed. During scraping, the stored muon orbit is shifted so that outliers in the phase space are lost on collimators during the first $20 \mu$ s after injection. In the 2nd half of the final E821 run, this technique resulted in an order of magnitude reduction in the losses during the $\omega_{a}$ fitting period.

The muon losses entry in Table IX arises mainly from the uncertainty in the possible difference between the average phases for stored and lost muons. For example, one source of muons, carrying a different phase and potentially lost at a higher rate, are those created after the momentum-selecting slit just upstream of the inflector. These muons, born from pion decay in that short region, have a different phase compared to those captured in the decay channel (the later muons did not go through the final dipole bend, which precesses the muon spin). In a 900-m long decay channel as we assume at FNAL, the population of muons born in the last turn into the storage ring will be essentially negligible and will be dwarfed fractionally by those born in the long AP2 decay channel. While this uncertainty can almost be eliminated, we include a small estimate here of $0.02 \mathrm{ppm}$ for smaller possible contributions to the fitting.

\section{Pileup}

The error due to pileup scales linearly with rate in each segment of the detectors. The effective size of the segment depends on the geometric extent of the shower. A simulation was used to demonstrate that the new W/SciFi calorimeters, having 20 or 35 independent segments, and a smaller Moliere radius, will provide an effective five-fold reduction in the intrinsic pileup. With no further improvements, and the proposed factor of up to 3 increase in data rate, the pileup error would be reduced by at least $3 / 5$ to $0.048 \mathrm{ppm}$. While we could accept this level of error, some improvement is desirable and achievable.

In the past, an artificial pileup spectrum was constructed from individual pulses in the data, then subtracted from the raw spectrum. In the pileup construction, it is necessary to use pulses with pulse heights below as well as above the hardware threshold. Because of 
the relatively high hardware threshold and limited storage of the E821 WFD system, those pulses below threshold were only found by searching during the relatively short period of continuous WFD digitization following the trigger generated by the presence of a large pulse above threshold. Consequently, the sample size for pileup events was limited and somewhat biased, since they had to always ride on the tails of larger pulses. In the new data WFD and data acquisition scheme, it will be possible to significantly improve the pileup construction process. Continuous digitization, with local software sorting of data streams including $T$ method, Pileup $T$-method and Q-method datasets, is anticipated. Pulses of all heights can be searched for independent of whether there is a nearby large pulse that fired a hardware trigger.

In E821, signals from four detector segments were combined before WFD digitization. Any mismatch in the relative timing of these signals can lead to variation in the pulse shape of the sum. In addition, the scintillator fiber in the calorimeters was strung radially, causing the pulse shape to depend slightly on the radial entrance position into the detector. These variations in the pulse shape hampered efforts to handle pileup, both in the fitting of two nearby peaks, and in the process of constructing the pileup spectrum. The pulse shape is expected to be more stable in the new design, because each segment will be individually digitized. In addition, the E821 WFDs were composed of two $200 \mathrm{MHz}$ ADCs that sampled the pulse shape out of phase and were later stitched together to form a $400 \mathrm{MHz}$ record. The upgraded experiment will employ single phase $500 \mathrm{MHz}$ WFDs based on a similar design that we have already successfully used in muon lifetime experiments at PSI.

The contribution of pileup to the error in $\omega_{a}$ for $E 821$ was divided into three components. The first two are correlated and add linearly. The third is not correlated so it is added in quadrature to the other two.

1. Pileup efficiency, $0.036 \mathrm{ppm}$. This is due to an estimated $8 \%$ uncertainty in the amplitude of the constructed pileup spectrum.

2. Pileup phase, $0.038 \mathrm{ppm}$. This is the error due to the uncertainty in the phase of the constructed pileup spectrum.

3. Unseen pileup, $0.026 \mathrm{ppm}$. This is the error due to pulses so small that they cannot be reconstructed and therefore they are not included in the pileup construction. 
We expect that the lower rate per detector segment in the new experiment, coupled with the new full-energy data stream will lead to a comprehensive pileup correction with minimal uncertainty. We assign up to $0.04 \mathrm{ppm}$ here to account for any difficulties in the anticipated analysis. As mentioned earlier, the $Q$ method is complementary to the traditional $T$ method and has different sources of systematic errors. The most significant difference is the effect of pileup--it is greatly reduced for the $Q$ method.

\section{Coherent Betatron Oscillations}

The average position and width of the stored beam can vary as a function of time as the beam alternately focuses and defocuses in the ring. This imposes an additional time structure on the decay time spectrum because the acceptance of the detectors depends on the position and width of the stored muon ensemble.

The CBO frequency lies close to the second harmonic of $\omega_{a}$, so the difference frequency $\omega_{\mathrm{CBO}}-\omega_{a}$ can be quite close to $\omega_{a}$, causing interference with the data fitting procedure and thereby causing a significant systematic error. This was recognized in analyzing the E821 data set from 2000. In the 2001 running period the electrostatic focusing field index was adjusted to minimize this problem. This greatly reduced the CBO systematic uncertainty. We will follow this tuning strategy again.

In addition, several efforts are underway to reduce the $\mathrm{CBO}$ effect even further. They include:

1. Improve the kicker pulse shape to better center the beam on orbit.

2. Use active RF schemes at very early decay times to reduce the amplitude of the CBO (see Appendix B).

3. Use an octupole $E$ or $B$ field at very early decay times to damp out the CBO amplitude (see Appendix B).

4. Increase the vertical size of the detectors. This reduces losses of positrons passing above or below the detector, reducing sensitivity of the detector acceptance to beam position and width.

The combined efforts should reduce the CBO uncertainty by a factor of 2 to 0.04 ppm. 


\section{E. Electric Field and Pitch Correction}

With a vertical magnetic field $B_{y}$ and radial electric field $E_{r}$, the precession frequency is given by

$$
\omega_{a}=-\frac{q}{m}\left[a_{\mu} B-\left(a_{\mu}-\frac{1}{\left(\gamma^{2}-1\right)}\right) \beta E_{r}\right] .
$$

If $B_{y}$ and $E_{r}$ vary with position, the time averages $\left\langle B_{y}\right\rangle$ and $\left\langle E_{r}\right\rangle$ should be used. At exactly the magic momentum the effect from $E_{r}$ is zero. Muons of slightly higher momentum $\delta p$ have an equilibrium orbit

$$
x_{e}=\frac{R_{o}}{1-n} \cdot \frac{\delta p}{p} .
$$

As they oscillate about this equilibrium orbit they experience a mean electric field $\left\langle E_{r}\right\rangle=$ $n\left(\beta B_{y} / R_{0}\right) x_{e}$ and their deviation from the magic momentum is proportional to $x_{e}$. This leads to a correction to $\omega_{a}$ proportional to $x_{\varepsilon}^{2}$. In this experiment $n$ is measured from the observed horizontal betatron frequency, and the distribution of muons with respect to $x_{e}$ is found from the modulation of counting rate by the rotation frequency of the muon bunch. The observed value of $\left\langle x_{e}^{2}\right\rangle$ was confirmed by simulation. The correction is $0.46 \mathrm{ppm}$.

With electric focusing, the plane in which the muon spin is precessing oscillates vertically, exactly following the oscillation of the muon momentum. When the orbit is inclined at angle $\psi$ to the horizontal, $\omega_{a}$ is reduced by the factor $\left(1-\frac{1}{2} \psi^{2}\right)$. If $\psi_{m}$ is the angular amplitude

of the vertical oscillation, the average over the ensemble of muons is $\left(1-\frac{1}{4}\left\langle\psi_{m}^{2}\right\rangle\right)$ where the brackets indicate an average over the muon population, $\left\langle\psi_{m}^{2}\right\rangle=n\left\langle y_{m}^{2}\right\rangle / r_{o}^{2}$ where $y_{m}$ is the amplitude of the vertical oscillation.

Information on $\left\langle\psi^{2}\right\rangle$ is obtained by simulation in which a representative set of muons is tracked around the ring from the inflector exit, via the kicker magnet, for many turns. The discrete quadrupole structure and aperture defining collimators are included as well as the calculated deviations from a pure quadrupole field. The pitch correction is $+0.29 \mathrm{ppm}$.

A combined (correlated) electric field and pitch correction uncertainty of 0.05 ppm was used in E821. We expect to improve on our knowledge of the electric field and pitch corrections by use of a new muon traceback system that can better image the beam motion versus time at a number of azimuthal positions around the ring. Furthermore, our simulation effort has improved, which is essential to some of these corrections. It is certainly feasible to reduce the uncertainty on these important corrections and we estimate a final combined error of $0.03 \mathrm{ppm}$. 
TABLE IX: The largest systematic uncertainties for the final E821 $\omega_{a}$ analysis and proposed upgrade actions and projected future uncertainties for data analyzed using the $T$ method.

\begin{tabular}{lc|lc}
\hline E821 Error & $\begin{array}{c}\text { Size } \\
{[\mathrm{ppm}]}\end{array}$ & Plan for the New $(g-2)$ Experiment & $\begin{array}{c}\text { Goal } \\
{[\mathrm{ppm}]}\end{array}$ \\
\hline Gain changes & 0.12 & Better laser calibration and low-energy threshold & 0.02 \\
Lost muons & 0.09 & Long beamline eliminates non-standard muons & 0.02 \\
Pileup & 0.08 & Low-energy samples recorded; calorimeter segmentation & 0.04 \\
CBO & 0.07 & New scraping scheme; damping scheme implemented & 0.04 \\
E and pitch & 0.05 & Improved measurement with traceback & 0.03 \\
\hline Total & 0.18 & Quadrature sum & 0.07 \\
\hline
\end{tabular}

\section{F. $\omega_{a}$ Systematic Uncertainty Summary}

Our plan of data taking and hardware changes address the largest systematic uncertainties and aims to keep the total combined uncertainty below $0.07 \mathrm{ppm}$. Experience shows that many of the "known" systematic uncertainties can be addressed in advance and minimized, while other more subtle uncertainties appear only when the data is being analyzed. Because we have devised a method to take more complete and complementary data sets, we anticipate the availability of more tools to diagnose such mysteries should they arise. Table IX summarizes this section.

\section{PARASITIC MEASUREMENT OF THE MUON ELECTRIC DIPOLE MO- MENT}

As has been discussed earlier, the magnetic moment of the muon is a sensitive probe for new, TeV scale, interactions. If the new interactions also contain CP violating phases, these phases will give rise to an electric dipole moment (EDM) of the muon. Contrary to the muon magnetic moment, there is no EDM expected in the Standard Model at a measurable level and thus any signal indicates new sources of CP violation beyond the Standard Model [85]. As noted in several places, the search for extra sources of CP violation is strongly motivated since the CP violation observed in the quark sector so far is insufficient to explain the matter dominance of the universe [86]. 
If the CP violating phases of the new interactions are independent of lepton flavor, the muon EDM will be related to the electron EDM by simple mass scaling. In this case, the current electron EDM limits rule out the ability to discover a muon EDM with the g-2 experiment proposed here. However, as discussed below, a muon EDM measurement performed with the proposed experiment could potentially extend the exclusion of flavor dependent $\mathrm{CP}$ violating interactions by 2 orders of magnitude.

The phenomenology of a muon EDM is described in detail in Ref. [87]. The muon dipole moment is related to the muon spin by

$$
\overrightarrow{d_{\mu}}=\frac{q}{2 m} \eta_{\mu} \vec{s}
$$

with $\eta_{\mu}$ playing a similar role to the $\mathrm{g}$ factor for the magnetic moment. The muon precession receives components from both anomalous magnetic moment, $a_{\mu}$, and $\eta_{\mu}$. At the magic momentum we have

$$
\begin{gathered}
\vec{\omega}_{a}=-\frac{q}{m} a_{\mu} \vec{B} \\
\vec{\omega}_{E D M}=-\frac{q}{2 m} \eta_{\mu}\left(\vec{\beta} \times \vec{B}+\frac{\vec{E}}{c}\right)=-\frac{\eta_{\mu}}{2 m c} \vec{F} .
\end{gathered}
$$

From this one sees that a muon aligned with the magnetic field will experience a torque that will act to tilt the precession plane away from the vertical direction by an angle

$$
\delta=\tan ^{-1}\left(\eta_{\mu} \beta / 2 a_{\mu}\right)
$$

One also sees from the above equation that the modulation in the vertical plane is sinusoidal and thus $90^{\circ}$ out of phase with the $a_{\mu}$ modulation.

The E821 collaboration has recently submitted for publication a measurement of the muon EDM by including an up-down asymmetry, offset by $90^{\circ}$ in the $\omega_{a}$ fit yielding an upper limit of $\left|d_{\mu}\right|<1.8 \times 10^{-19} \mathrm{ecm}[87]$ which is a factor of 5 improvement over the previous best limit [88]. Results of the fit are displayed in Fig. 31. The measurement was performed in part using straw tube tracking detectors [89] that were designed to determine the muon beam distribution within the storage ring and instrumented in front of one of the 24 calorimeter stations. 

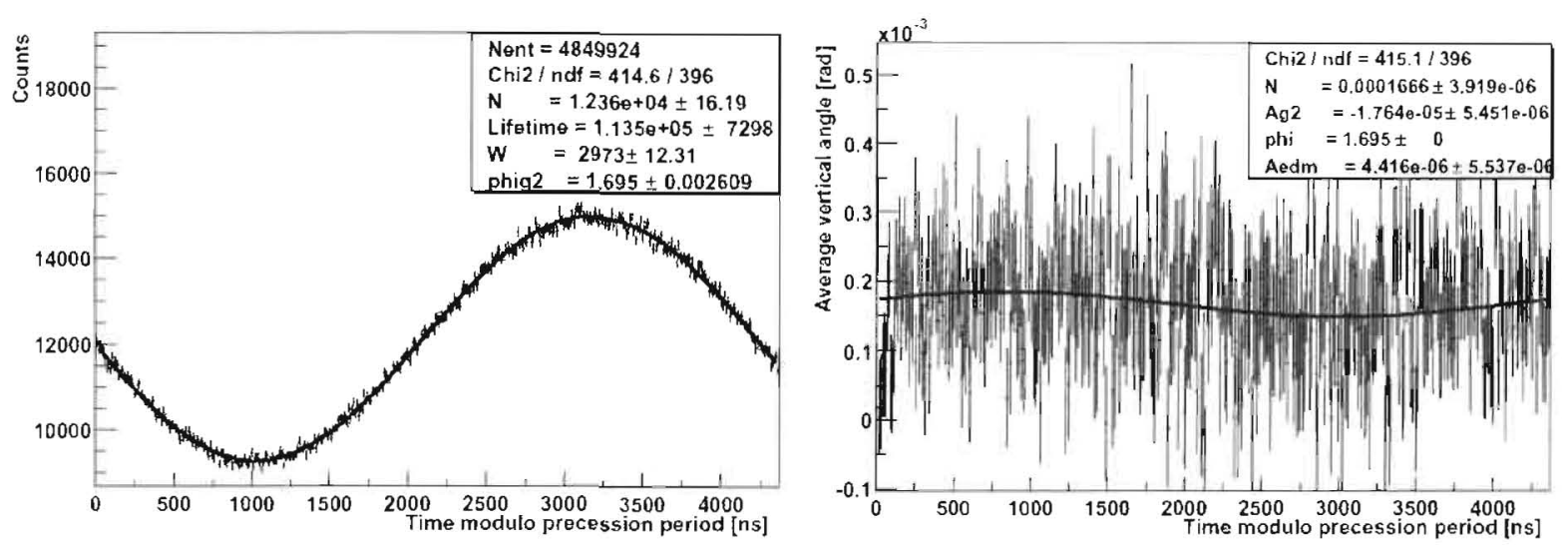

FIG. 31: Data recorded by the E821 traceback system. The left distribution is the number of tracks versus time modulo the precession frequency. The right distribution is the average vertical angle of the tracks versus time also modulo the precession frequency.

\section{A. E821 Traceback System}

A replication of the E821 traceback system is required for the proposed g-2 measurement. The available statistics for a muon EDM measurement could be greatly increased by instrumenting several more calorimeter stations with tracking capability. This can be accomplished by placing the straw tracking chambers inside the vacuum chambers in the scallop region in front of the calorimeters.

A similar number of channels to the E821 traceback system can be mounted on extra vacuum ports that exist and are not instrumented in most scallop regions as indicated in Fig. 33. A final layer can be placed outside the vacuum, directly in front of the calorimeters. While 23 additional calorimeter stations exist, several of them do not have a clear line of sight to the muon beam due to quadrapole magnets or other instrumentation so that instrumenting all stations with tracking capabilities may not be feasible.

Besides increasing the statistics for an EDM measurement, increased tracking instrumentation will allow the beam profile to be measured at several locations around the ring. This will be very useful input to beam simulation and it is expected to lead to a reduction in the errors associated with the electric field pitch corrections from $0.05 \mathrm{ppm}$ to $0.03 \mathrm{ppm}$. 


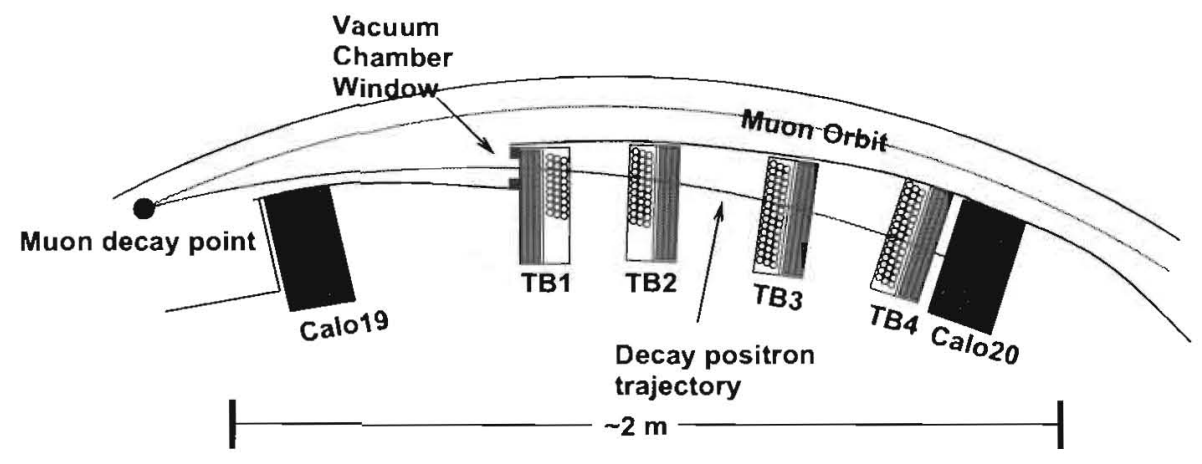

FIG. 32: Top view of the E821 traceback straw tracking system.

\section{B. Improved Traceback System}

A replication of the E821 traceback system is required for the proposed $(g-2)$ measurement. The available statistics for a muon EDM measurement could be greatly increased by instrumenting more calorimeter stations with tracking capability. This can be accomplished by placing the straw tracking chambers inside the vacuum chambers in the scallop region in front of the calorimeters. In this position, the acceptance of the chambers is greatly increased compared to that of $\mathrm{E} 821$.

A similar number of channels to the E821 traceback system can be mounted on extra vacuum ports that exist and are not instrumented in most scallop regions as indicated in Fig. 33. A final layer can be placed outside the vacuum, directly in front of the calorimeters. While 23 additional calorimeter stations exist, several of them do not have a clear line of sight to the muon beam due to quadrupole magnets or other instrumentation so that instrumenting all stations with tracking capabilities may not be feasible.

An $R \& D$ program is underway at Fermilab to demonstrate the feasibility of such a tracking system. This program builds largely on experience gained from the CKM R\&D program to develop straws in vacuum [90] and current efforts to develop straws for improved instrumentation of the meson test beam area (MTest). Fermilab is also developing a Geant 4 based simulation to determine the overall requirements of the system such as the allowed material budget, the effects of pileup and other beam backgrounds, and the effects of material, such as the quadrupoles, that exist between the muon beam and the location of the tracking stations. 


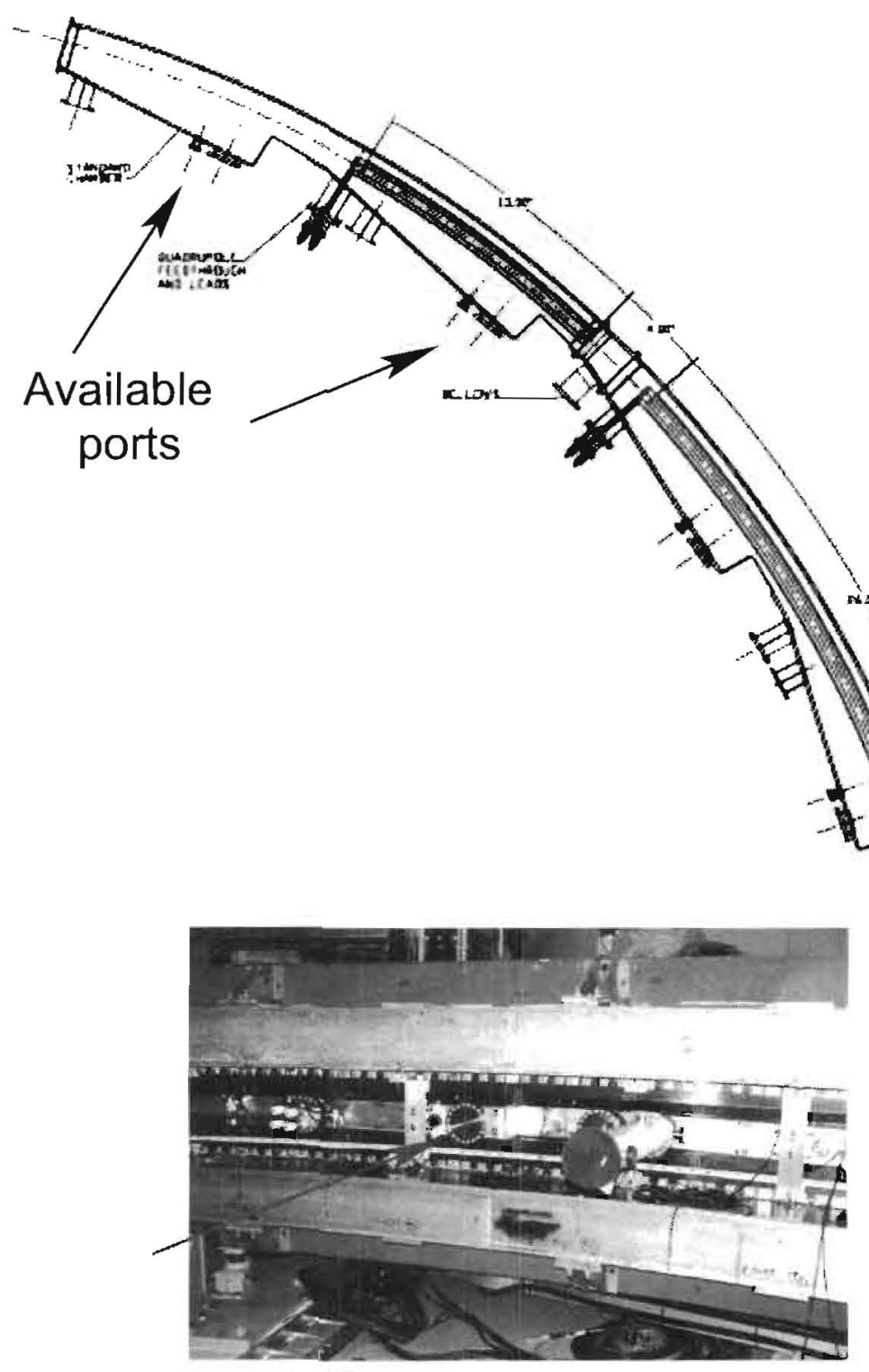

FIG. 33: Top: Beam Pipe schematic indicating available vacuum ports where straw tracking chambers can be placed. Bottom: picture of available ports. 


\section{MANPOWER, COST ESTIMATE AND SCHEDULE}

\section{A. Manpower}

We have formed a new collaboration to carry out this measurement, which represents a healthy ratio of former E821 collaborators along with strong new institutions. Fortunately, much of the expertise built up in E821 is represented and still available to us. We still have senior experts in the collaboration who cover all of the different technical areas. New institutions bring creative input and additional technical capabilities. These groups include the host laboratory--Fermilab--and new university groups at James Madison, Kentucky, Massachusetts, Michigan, Regis, and Virginia. New international groups include KEK and Osaka from Japan, KVI-Groningen from The Netherlands, Frascati from Italy, and PNPI from Russia. The Muons Inc. team has also joined the effort. In some cases, experienced younger E821 collaborators are associated with their new institutions. The names listed on the proposal masthead are mainly senior physicists. As the experiment develops, we expect postdocs and students to sign on; indeed, with approval, we will immediately begin a vigorous campaign to enlist the talents of young people. We are proud that E821 offered a rewarding experience for dozens of postdocs and graduate students and we intend to welcome young physicists in the new effort.

\section{B. Cost Estimate}

The New $(g-2)$ Experiment is based on the well understood efforts associated with the BNL E821 experiment. We intend to utilize a large fraction of available custom instrumentation from that effort, principally the storage ring magnet and its internal and supportive subsystems. New to the FNAL experiment will be the beam delivery scheme and improvements in the precession and field measurement instrumentation that will permit reduction of systematic errors.

The tasks and costs fall into three main areas.

\section{Beamline}

The transformation of the existing FNAL rings and accelerator complex toward hosting experiments at the intensity frontier involves upgrades to the Booster, transfer lines 
into and out of the Recycler, RF upgrades to the Recycler, modifications of the APO pulsed dipole magnet, additional quadrupoles in the AP2 transfer line, an extraction port from the Debuncher, the opening of certain fixed apertures in the AP3 transfer line, and finally a new short beamline stub into the muon storage ring. Many of the items are required for the approved Mu2e experiment and would be required for any other high-intensity experiment based on the $8-\mathrm{GeV}$ proton beams. We identify those items that are unique to the New $(g-2)$ Experiment in our Table of costs. The costs for some entries have only been estimated at this time. We note that none are risky developments and none require any high-degree of technical design.

\section{The Storage Ring}

The superconducting storage ring, its power supplies, internal subsystems such as the vacuum system, electrostatic quadrupoles, kicker, pickup electrodes, fiber monitors, and the external FODO beamline and beamline stub into the ring all exist. They are available to us from BNL under laboratory and DOE agreements (which will have to be formalized). These items must be packaged and transported from BNL to FNAL and reassembled. BNL engineers have estimated the costs for the ring disassembling, shipping, and reassembly at FNAL. FNAL experts estimated the costs for a new temperature-stable building and for necessary cryo and power connections. Because the transport of such an instrument carries risk, we included a $100 \%$ contingency for this effort. The re-assembly - mostly labor-is assigned at $50 \%$ contingency.

\section{Detector and Measuring Systems}

The magnetic field measuring system will be upgraded. It is of relatively modest cost and will be provided for by the collaboration. A new set of electromagnetic calorimeters, their optical readout and waveform digitizers, are also required. Prototypes have been built and bids for production quantities have been made for the detector. Waveform digitizers have been designed and built by our collaboration and costs for a new system are relatively well understood. The data acquisition system will be new. Straw detectors and their readout are required for each calorimeter station. We estimate that most of the costs for the detector and measuring systems will come from collaborating institutes with support from their respective funding agencies through supplemental support. We also plan for support in this area from our international collaborators. 
Figure 34 shows tables that summarize the cost estimates as we now know them. Below the figure is a set of notes referring to the individual entries. Many of the local FNAL estimates have been made by experts, but they are not based on detailed studies. Estimated contingencies and overhead appropriate to the entry and risk are included. Notes provided below the table indicate sources and other information where available. If Stage 1 approval is awarded, will we begin a thorough design and cost review for the beamline and ring relocation associated costs, which drive the budget. It is our intention to keep the total project costs as lean as possible, while still providing a high-impact, state-of-the-art precision experiment, that can be built and run in the near-term.

We divide the cost tables into two general categories. In the shaded yellow box are items required for a FNAL based high-intensity proton program (many of these are in progress now). We believe these items will have longer term benefits, from more smoothly leading up to Mu2e operation, to opening the possibility of other experiments not yet proposed, which will require custom beam structures and high intensity. Specific to the New $(g-2)$ Experiment are items shaded in green. The $(g-2)$ Table includes a column specific to DOE-HEP (mainly beamline and ring relocation) and one that we expect to obtain from support to NSF user groups and from our international partners. The numbers in the tables include contingency. 


\begin{tabular}{|c|c|c|c|c|c|}
\hline Subsystem & M\&S & Labor & Contingency & FNAL & Note \\
\hline Booster15Hz & 1517 & 373 & $30 \%$ & 2457 & 1 \\
\hline Inj $8 \mathrm{GeV}$->RR & 0 & 0 & & 0 & 2 \\
\hline Recycler Ring RF & 1700 & 1322 & $17 \%$ & 3536 & 3 \\
\hline Ext RR $>P$ P & 220 & 491 & $25 \%$ & 889 & 4 \\
\hline Trans.Line - RR2P1 & 732 & 1311 & $35 \%$ & 2759 & 5 \\
\hline P1,P2, Inj/Ext DA/AA & 200 & 329 & $100 \%$ & 1058 & 6 \\
\hline TOTALS & & & & 10699 & \\
\hline
\end{tabular}

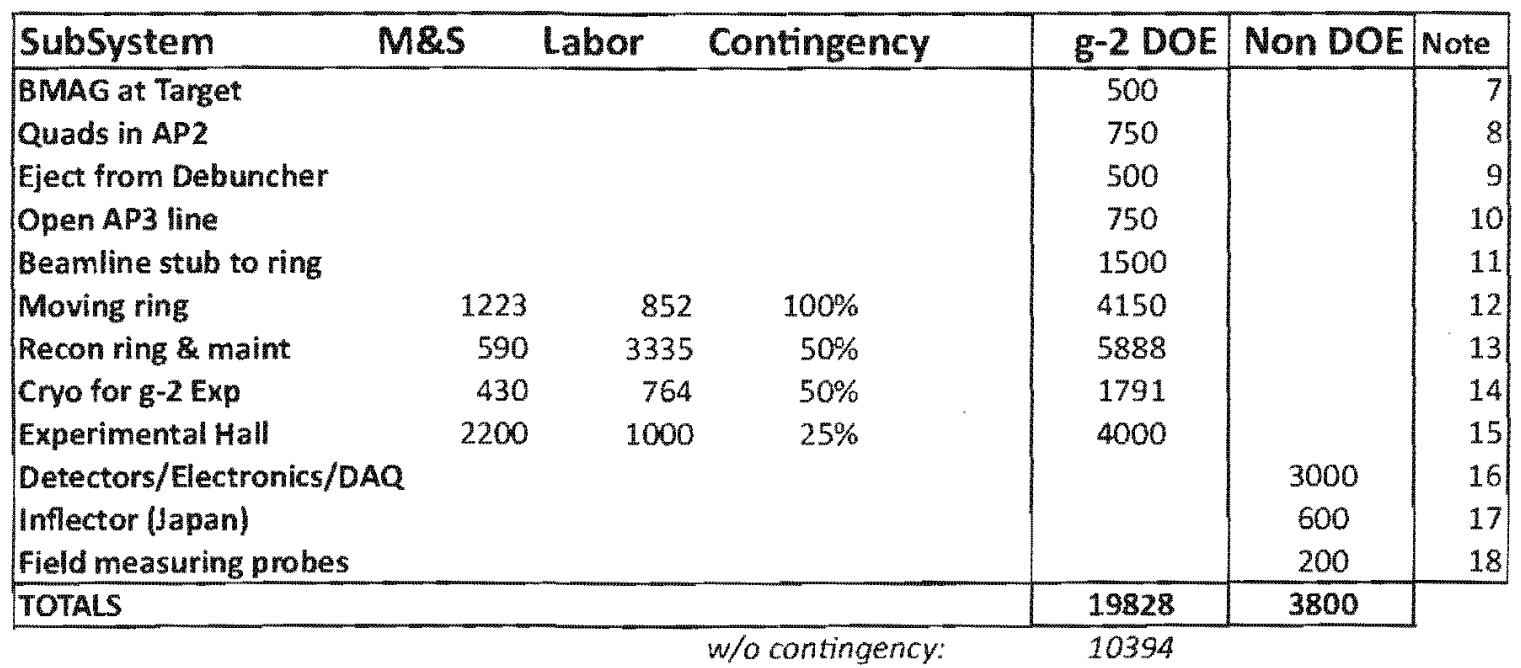

FIG. 34: Preliminary Cost Estimate for the New $(g-2)$ Experiment. Rates used for labor estimates were $\$ 228 \mathrm{k} / \mathrm{FTE}$ for engineering, $\$ 130 \mathrm{k} / \mathrm{FTE}$ for technician effort, and $\$ 135 \mathrm{k} / \mathrm{FTE}$ for drafting/design. The yellow table is generic for a high-intensity proton program at FNAL (including the Mu2e Experiment.) The green table is specific to the New $(g-2)$ Experiment. Costs in the table include contingencies.

1. FNAL AD/RF technical note

2. Part of the NOvA project; required for $(g-2)$

3. FNAL AD/RF technical note

4. FNAL AD/EE technical note

5. Estimate based on NOvA project, Boo2RR line

6. Kicker magnet \& PS; FNAL AD/EE 
7. Estimate for new magnet

8. Estimate to bring quads from BNL, install; stands, etc.

9. Estimate to move transfer line to AP3 from $A C C$ to DEB ring

10. Estimate; not designed

11. Estimate; not designed

12. Shipping based on commercial quotes and BNL engineer estimates of labor; includes $100 \%$ contingency

13. Assembly based on BNL manpower and time estimates; includes $50 \%$ contingency

14. FNAL AD/Cryo presentation $9 / 08$

15. FNAL FESS; $25 \%$ contingency; includes EDIA and indirect

16. Commercial Quotes plus experience building similar systems

17. Tolkin Quote in Japan

18. Collaboration estimate

\section{Schedule}

Assuming approval of this proposal, we outline the technically driven schedule. If we obtain CD-0 in mid-2009, then work can begin over the next 12 months - in parallel-on three main fronts:

1. Detailed beamline calculations and design of the stub into the storage ring. Final building plans and final citing decisions.

2. Inventory of all BNL equipment that will be available for the New $(g-2)$ Experiment. Detailed procedure for decommissioning the storage ring and a complete shipping plan.

3. R\&D for the calorimeter readout using SiPMs, tests with a new full-scale prototype, and R\&D for in-vacuum straw detectors. A design and prototype for the out of vacuum straw chambers. 
With construction funding available, it will take approximately 1 year to build the new ring housing at FNAL, 1 year to install and make operative the storage ring, and 1 year (with cryo and power) to shim the magnet to high field uniformity. This sequence drives the schedule and permits experiment commissioning in the mid-2013 (or, three years following funding). In parallel, the beamline modifications will be made and the detector systems will be built and tested. As part of our plan, selected beamline rate tests can be made earlier with modest impact on the main program.

\section{PLANNED R\&D EFFORTS}

While the New $(g-2)$ Experiment is designed largely following the successful existence proof from BNL E821, it is a challenge to increase the statistics by more than a factor of 20 and reduce the net systematics uncertainties by a fact of 3 . Here, we outline some of the main anticipated R\&D efforts, where an expedient effort can influence the final design and cost reliability. Many other R\&D efforts are presented in previous sections.

- Lithium Lens Pulsing at $18 \mathrm{~Hz}$. The existing antiproton target and lithium lens system are envisioned for use by our experiment. The fundamental difference between the current operation with antiprotons and the demands for $(g-2)$ are higher repetition rate $(0.5 \mathrm{~Hz} \rightarrow 18 \mathrm{~Hz})$, but at lower proton energy $(120 \rightarrow 8 \mathrm{GeV})$. The lithium lens pulsing system must be studied and possibly tested to determine if it can operate under these conditions.

- Decay Channel Optimization. Table VI lists several upgrades to significantly improve the muon storage efficiency per proton. Several others are under consideration. Detailed studies are required to make the most complete and cost-effective choices. These studies include: optimization of the beam focus on the target; optimization of the Li lens optics; complete an end-to-end decay simulation model of AP2 beam line including additional quadrupole elements; optimization of the optics for maximum magic muon collection; and, simulate the full transport chain through the inflector and study transmission into ring with closed/open inflector versions and beam kicker variants. 
- Beamline test program. FNAL offers the attractive option that key factors contributing to the anticipated stored muon rate can be verified in beam tests in the near future. While the final experiment needs the dedicated pulse structure, the foreseen intensity tests can be performed with booster batches brought on target through the current main injector beamlines. A test program including required instrumentation will be designed.

- Half-Length Kicker Plate Test. A prototype kicker plate system exists in a straight vacuum chamber at BNL. To reduce the inductance of the kicker LCR pulse-forming network, we can build a half-length kicker system and test it with existing equipment. The outcome could provide a relatively straight-forward method of improving the fast muon kicker waveform, and thereby increasing the muon storage efficiency. This modification would require the construction of three additional kicker modulators to drive the shorter kicker sections, and a re-working of the modulator housings.

- Tracking of the Magnetic Field. The measurement of the average magnetic field in experiment E821 relied on continual measurements with fixed probes above and below the muon storage region. These probes measured the field with respect to measurements with the field trolley, which was calibrated with a plunging probe and an absolute field probe. In E821 about half of the fixed probes could not be used effectively, due to their proximity to the joints in the precision pole pieces and yoke and the resulting field inhomogeneity. A detailed $R \& D$ effort to re-optimize the fixed probe configuration around the storage ring would increase the number and effectiveness of the fixed probes, and should thereby be expected to improve the tracking uncertainty in the dipole field between field measurements with the trolley. The tracking of higher multipoles should benefit greatly from the placement of probes besides the muon storage region. These probes would need to be placed in vacuum near the horizontal quadrupole plates, and a system that can operate in this environment would need to be developed.

- Large-Scale W/SciFi Prototype and SiPM Readout. We intend to build a near full-scale calorimeter prototype, which can be readout by both conventional PMTs and new SiPM arrays. The comparison for pileup, gain stability, easy of assembly, and the 
final segmentation will be part of the investigation. As with past prototypes, we would use the FNAL Meson Test area for beam related tests.

- In-vacuum Straw Chambers. To make higher precision images of the beam dynamics, monitor muon losses, and to enable a high-statistics measurement of the muon electric dipole moment, we plan to deploy a set of wire chambers on the face of each calorimeter with a paired set placed directly upstream in vacuum. A test setup is underway now at FNAL to investigate this concept and work out the technical details of placing the straws in vacuum.

\section{SUMMARY OF THE REQUEST}

We propose to improve the current measurement of the muon anomalous magnetic moment $a_{\mu}$ by a factor of 4 . Combined with expected progress on the theoretical value, a $0.14 \mathrm{ppm}$ result will nearly triple the sensitivity of the measurement to standard model extensions. The present suggestion from the final $\mathrm{E} 821$ result of a standard model violation may be contradicted, or verified, but whether the new result agrees with the standard model or not, it will place important constraints on candidate models of new physics- especially in the context of eagerly anticipated data from the LHC.

In this proposal, we have identified a timely and cost-effective method of using the FNAL beam complex in the post-Tevatron era to produce custom, high-intensity bunches of muons that can be injected into the existing superconducting storage ring. While a major task will be the relocation of the ring from Brookhaven to FNAL, it is work carrying relatively low risk. The proposed experiment builds on the considerable expertise developed over more than 20 years in the design, construction, data taking and analysis efforts from E821. The collaboration has been re-invigorated with many strong new groups who will work in concert with experts from the previous measurement. The physics motivation is compelling and promises to continue to provide unique insight into fundamental questions in high-energy physics. 
[1] J. Schwinger, Phys. Rev. 73, 416 (1948).

[2] G.W. Bennett, et al., (Muon ( $g-2$ ) Collaboration), Phys. Rev. D 73, 072003 (2006).

[3] Eduardo de Rafael Present Status of the Muon Anomalous Magnetic Moment; To appear in the proceedings of 14th High-Energy Physics International Conference in Quantum Chromodynamics (QCD 08), Montpellier, France, 7-12 Jul 2008. e-Print: arXiv:0809.3085.

[4] G. Charpak, F.J.M. Farley, R.L. Garwin, T. Muller, J.C. Sens, and A. Zichichi, Phys. Lett. $1,16(1962)$.

[5] J. Bailey et al., Nuovo Cimento A9, 369 (1972).

[6] J. Aldins, S.J. Brodsky, A.J. Dufner and T. Kinoshita, Phys. Rev. D 1, 2378 (1970).

[7] J. Bailey et al., Nucl. Phys. B150, 1 (1979).

[8] The $g-2$ Collaboration: R.M. Carey et al., Phys. Rev. Lett. 82, 1632 (1999).

[9] The $g-2$ Collaboration: H.N. Brown et al., Phys. Rev. D 62, 091101 (2000).

[10] The $g-2$ Collaboration: H.N. Brown et al., Phys. Rev. Lett. 86, 2227 (2001).

[11] The $g-2$ Collaboration: G.W. Bennett et al., Phys. Rev. Lett. 89, 101804 (2002); Erratumibid. $89,129903(2002)$.

[12] The g-2 Collaboration: G.W. Bennett et al., Phys. Rev. Lett. 92, 161802 (2004)x

[13] W. Liu et al, Phys. Rev. Lett. 82, 711 (1999).

[14] K. Hagiwara et al, Phys. Rev, D66, 010001 (2002).

[15] R. McNabb et al, Nucl. Inst, Meth., in press, 2009.

[16] T. Kinoshita and M. Nio, Phys. Rev. D 73, 053007 (2006).

[17] T. Aoyama, M. Hayakawa, T. Kinoshita, M. Nio, and N. Watanabe, Phys. Rev. D 78, 053005 (2008).

[18] D. Hanneke, S. Fogwell and G. Gabrielse, Phys. Rev. Lett. 100, 120801 (2008),

[19] James P. Miller, Eduardo de Rafael and B. Lee Roberts, Rept. Prog. Phys. 70, 795-881, 2007.

[20] F. Jegerlehner, http:/www.Inf.infn,it/conference/phipsi08/International Workshop on e+ecollisions from Phi to Psi, Frascati, 7-10 April, 2008.

[21] M. Passera, W.J. Marciano and A. Sirlin, Phys. Rev. D 78, 013009 (2008).

[22] See: http:/ www.infinfn.it/wg/sighad/ report of the "Working Group on Rad. Corrections 
and MC Generators for Low Energies" (Radio MontecarLow) to be published on Euro. Phys.Jour. C.

[23] K. Hagiwara, A.D. Martin, D. Nomura and T. Teubner, Phys. Lett. B649 173 (2007).

[24] M. Davier, hep-ph/0701163v2, Jan. 2007.

[25] F. Jegerlehner, Acta Phys. Polon. B 38, 3021 (2007) [arXiv:hep-ph/0703125].

[26] A. Höcker and W.J. Marciano, The Muon Anomalous Moment, Particle Data Tables, Phys. Lett. B667 1 (2008).

[27] Zhiqing Zhang, Muon $g-2$ : a mini review, arXiv:0801.4905v1 [hep-ph], Jan. 2008.

[28] KLOE collaboration (F. Ambrosino et al.), Phys. Lett. B669, 223-228 (2008).

[29] M. Davier, TAU'08, Novosibirsk, 22-25 September 2008.

[30] R. Alemany, M. Davier and A. Höcker, Eur.Phys.J. C2 123 (1998).

[31] M. Davier and A. Höcker, Phys. Lett.B435, 427 (1998).

[32] M. Davier, S. Eidelman, A. Höcker, and Z. Zhang, Eur. Phys J. C 27497 (2003). See K. Hagiwara, A.D. Martin, D. Nomura, and T. Teubner Phys. Lett. B557, 69 (2003) for an independent analysis of the $e^{+} e^{-}$data.

[33] Joaquim Prades, Eduardo de Rafael and Arkady Vainshtein, arXiv:0901.0306v1 [hep-ph] Jan. 2009.

[34] Arkady Vainshtein, private communication.

[35] Topical Workshop on The Muon Magnetic Dipole Moment; Oct. 2007 School of Physics and Astronomy, The University of Glasgow. See: www.ippp.dur.ac.uk/old/MuonMDM/.

[36] T. Blum, Phys. Rev. Lett. 91, 052001-1 (2003).

[37] T. Blum, Nucl. Phys. Proc. Suppl. 129, 904-906,2004, and arXiv hep-lat/0310064,

[38] T. Blum, private communication, February 2009.

[39] T. Kinoshita and W.J. Marciano in Quantum Electrodynamics (Directions in High Energy Physics, Vol. 7), ed. T. Kinoshita, (World Scientific, Singapore, 1990), p. 419.

[40] Andrzej Czarnecki and William J. Marciano, Phys. Rev. D64 013014 (2001).

[41] M. Davier and W.J. Marciano, Ann. Rf. Nucl. Part. Phys. 54, 115 (2004).

[42] The articles listed in the SPIRES citations to Ref. [10] contain many different models beyond the standard model.

[43] E. Eichten, et al., Phys. Rev. Lett. 45, 225 (1980), K. Lane, arXiv hep-ph/0102131, 2001.

[44] T. Appelquist and B. A. Dobrescu, "Universal extra dimensions and the muon magnetic 
moment," Phys. Lett. B 516 (2001) 85 [arXiv:hep-ph/0106140].

[45] Friedrich Jegerlehner, The Anomalous Magnetic Moment of the Muon, Springer Tracts in Modern Physics Vol. 226.

[46] D. W. Hertzog, J. P. Miller, E. de Rafael, B. Lee Roberts and D. Stöckinger, arXiv:0705.4617 [hep-ph].

[47] J. M. Smillie and B. R. Webber, JHEP 0510 (2005) 069 [arXiv:hep-ph/0507170].

[48] B. C. Allanach et al., Proc. of the APS/DPF/DPB Summer Study on the Future of Particle Physics (Snowmass 2001) ed. N. Graf, Eur. Phys. J. C 25 (2002) 113 [eConf C010630 (2001) P125].

[49] R. Ruiz de Austri, R. Trotta and L. Roszkowski, JHEP 0605 (2006) 002 [arXiv:hepph/0602028]; JHEP 0704 (2007) 084 [arXiv:hep-ph/0611173]; JHEP 0707 (2007) 075 [arXiv:0705.2012]; B. C. Allanach, C. G. Lester and A. M. Weber, JHEP 0612 (2006) 065; B. C. Allanach, K. Cranmer, C. G. Lester and A. M. Weber, JHEP 0708, 023 (2007); J. R. Ellis, S. Heinemeyer, K. A. Olive, A. M. Weber and G. Weiglein, JHEP 0708 (2007) 083; S. Heinemeyer, X. Miao, S. Su and G. Weiglein, JHEP 0808, 087 (2008).

[50] J. R. Ellis, K. A. Olive, Y. Santoso and V. C. Spanos, Phys. Lett. B 565176 (2003); John Ellis, Keith A. Olive, Yudi Santoso, and Vassilis C. Spanos, Phys. Rev. D71 095007 (2005), and references therein.

[51] N. Arkani-Hamed, G. L. Kane, J. Thaler and L. T. Wang, JHEP 0608, 070 (2006) [arXiv:hep$\mathrm{ph} / 0512190]$.

[52] R. Lafaye, T. Plehn, M. Rauch and D. Zerwas, arXiv:0709.3985 [hep-ph].

[53] C. F. Berger, J. S. Gainer, J. L. Hewett, B. Lillie and T. G. Rizzo, ar Xiv:0712.2965 [hep-ph].

[54] M. Alexander, S. Kreiss, R. Lafaye, T. Plehn, M. Rauch, and D. Zerwas, Chapter 9 in M. M. Nojiri et al., arXiv:0802.3672 [hep-ph].

[55] G. Ross and M. Serna, arXiv:0704.1248 [hep-ph].

[56] S. P. Martin and J. D. Wells, Phys. Rev. D 67, 015002 (2003) [arXiv.hep-ph/0209309].

[57] C. F. Berger, J. S. Gainer, J. L. Hewett and T. G. Rizzo, arXiv:0812.0980 [hep-ph].

[58] For information about the Fermilab Proton Plan, see: http://www-accel-proj.fnal.gov/Proton_Plan/index.shtml.

[59] C. Bhat, J. MacLachlan, "RF Requirements for Bunching in the Recycler for Injection into the g-2 Ring," Beams-doc-3192 (28 Aug 2008). 
[60] Russ Alber, Internal Memo, 25 Aug 2008. See Beams-doc-3197 (4 Sep 2008),

[61] J. Theilacker, Internal Memo, 21 Aug 2008.

[62] F. Krienen, D. Loomba and W. Meng, Nucl, Inst. and Meth. A 283, 5 (1989).

[63] A. Yamamoto, et al., Nucl. Instrum. and Methods Phys. Res. A491 23-40 (2002).

[64] G.T. Danby, et al., Nucl. Instr. and Methods Phys. Res. A 457, 151-174 (2001).

[65] E821 - Technical Design Report

[66] R. Prig! et al, Nucl. Instrum. Meth. A374, 118 (1996).

[67] R. Prigl, PhD Thesis, U. Heidelberg (1994).

[68] A.P. Grossmann, PhD Thesis, U. Heidelberg (1998).

[69] S.I. Redin, PhD Thesis, Yale University (1999).

[70] H. Deng, PhD Thesis, Yale University (2002).

[71] G. Danby et al, Nucl. Instrum. Meth. A457, 151 (2002).

[72] A. Yamamoto et al, Nucl. Instrum. Meth. A491, 23 (2002).

[73] E. Efstathiadis et al, Nucl. Instrum. Meth. A496, 8 (2003).

[74] X. Fei, V.W. Hughes, and R. Prigl, Nucl. Instrum. Meth. A394, 349 (1997).

[75] Superintendent of Documents, LORAN-C User's Handbook, U.S. Government Printing Office \#050-012-00331-9, 1992.

[76] P.J. Mohr and B.N. Taylor, Rev, Mod. Phys. 72, 351 (2000).

[77] S. I. Redin et al, Nucl. Instrum. Meth. A 473, 260 (2001).

[78] P. Neumayer, Diploma Thesis, U. Heidelberg (1999).

[79] Paul Scherrer Instutut Experiment R-99-07, A Precision Meastrement of the Positive Muon Lifetime using a Pulsed Muon Beam and the flan Detector.

[80] Paul Scherrer Instutut Experiment R-97-05, Precision Measurement of Singlet $\mu$ p Capture in Hydrogen.

[81] D.W. Hertzog et al., Nucl. Instrum. Methods Phys. Res. A294, 446 (1990).

[82] S.A. Sedykh et al., Nucl. Inst. Methods Phys. Res. A455 346 (2000)

[83] J. Ouyang et al., Nucl. Instrum. Methods Phys. Res. A374, 215 (1996).

[84] For example, see C. Fabjan, "Calorimetry in high-energy physics", Experimental Techniques in High-Energy Nuclear and Particle Physics, 2nd Ed., World Scientific Publishing Co., T. Ferbel, Ed., (1991), 257.

[85] M. Raidal et al.,Eur. Phys. J. C57, 13 (2008). 
[86] P. Huet, E. Sather, Phys. Rev. D51 379 (1995).

[87] G.W. Bennett et al. (Muon (g-2) Collaboration), arXiv:0811.1207v1, submitted to Phys. Rev. D.

[88] J. Bailey et al., j. Phys. G4, 345 (1978).

[89] M. Sossong et al., submitted to Nucl. Int. Meth. A

[90] C. Kendziora et al., Fermilab-Pub-02-241-E, submitted to Nucl. Int. Meth. A

[91] J.L. Flowers, B.W. Petley, M.G. Richards, Metrologia 30 (1993) 75; K. Jackowski, M. Jaszunski, B. Kamienski, M. Wilczek, J. Magn. Reson. 193 (2008) 147.

[92] N.V. Mokhov, "The Mars Code System User's Guide", Fermilab-FN-628 (1995); N.V. Mokhov, S.I. Striganov, "MARS15 Overview", Fermilab-Conf-07/008-AD (2007), in Proc. of Hadronic Shower Simulation Workshop, Fermilab, September 2006, AIP Conf. Proc. 896, pp. 50-60 (2007); http:/www-ap.fnal.gov/MARS/

[93] U. Rohrer, http://pc532.psi.ch/optics.htm

[94] V. Lebedev, http:/www-bdnew fnal.gov/pbar/organizationalchart/lebedev/OptiM/optim.htm

[95] Y.K. Semertzidis, Nucl. Instrum. Methods Phys. Res, A503 458-484 (2003)

[96] This section was adapted from Yuri F. Orlov and Yannis K. Semertzidis, Muon $(g-2)$ Note 431, January 21, 2003.

[97] This section was adapted from a preprint by 1.A.Koop, A.V.Otboev, E.A.Perevedentsev, P.Yu.Shatunov and Yu.M.Shatunov of the Budker Institute for Nuclear Physics, Novosibirsk.

[98] L.D.Landau, E.M.Lifshitz; "Mechanics", Moscow "Nauka" (1988)

[99] A.N.Dubrovin, "MERMAID user guide", Novosibirsk (1994).

[100] E.A.Perevedentsev et al., Preprint BINP, Novosibirsk (1980)

[101] Saint-Gobain Crystals and Detectors, 12345 Kinsman Road, Newbury, OH 44065.

[102] Marketech International, 4750 Magnolia Street, Port Townsend, WA 98368.

[103] At Fermilab, the beam energy of the Booster is typically quoted as " $8 \mathrm{GeV}$ " and refers to the kinetic energy. The total energy per incident proton is thus $8.938 \mathrm{GeV}$, and the momentum per proton is $8.889 \mathrm{GeV} / \mathrm{c}$.

[104] Other small uncertainties raise the cited total to $0.21 \mathrm{ppm}$. These additional errors are upper limits that will improve with increased statistics of the sample and thus will not change our projections significantly. 


\section{APPENDIX A: MUON COLLECTION AND TRANSPORT BEAMLINE}

\section{Beamline Overview}

Fig. 35 compares the lattice function of the E821 beamline with the AP2 beamline at FNAL. The E821 beamline is comprised of five sections: the pion collection and pion spectrometer, the matching section, the decay channel, the muon spectrometer, and the matching section to the storage ring. The optical constraints determine an essentially unique tune for the beamline. At slits $\mathrm{K} 1 \mathrm{~K} 2$ the initial pion momentum is selected in a $0.5 \%$ bin around $p_{\pi}=\alpha p_{\text {magic }}$, where $p_{\text {magic }}=3.094 \mathrm{GeV} / \mathrm{c}$ is the magic muon momentum. To collect magic muons from forward decay in the pion CMS, a value of $\alpha=1.005$ is required. However, given the short length of $88 \mathrm{~m}$ of the decay section compared to the relativistic pion decay length of $173 \mathrm{~m}$, this would lead to an insufficient pion suppression at the muon selecting slit $\mathrm{K} 3 \mathrm{~K} 4$ and $F_{\pi}=1 \times 10^{-5}$ pions per proton would be transmitted into the $(g-2)$ ring. As the resulting hadronic Hash leads to an unacceptable background in the experiment, E821 was operated at $\alpha=1.017$, where the pion flash is reduced to $F_{\pi}=2 \times 10^{-3}$ at the cost of reducing the number of stored muons by about a factor of 3 . The straight FODO decay section has a spacing of $6.2 \mathrm{~m}$ between 11 quadrupoles (4-inch diameter aperture) operating at moderate fields of $1.8 \mathrm{kG}$. The overall unnormalized channel acceptance up to the inflector is $A_{x}=42 \pi m m-m r a d$ and $A_{y}=56 \pi m m-m r a d$, the combined ring-inflector acceptance is similar. 


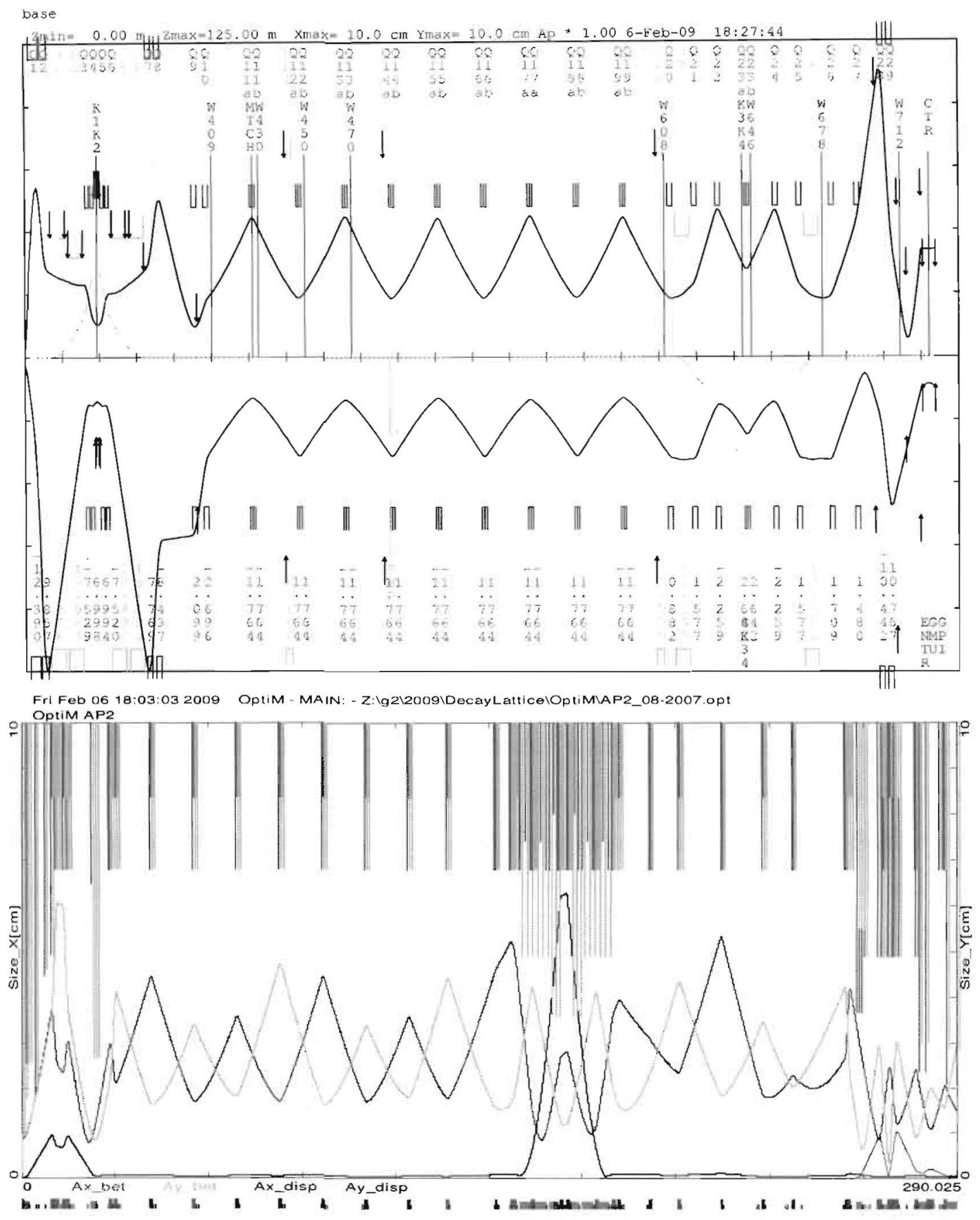

FIG. 35: Beam envelopes and dispersion for E821 and FNAL AP2 beamline from TRANSPORT [93]. (top: y-plane, bottom: x-plane) and OptiM [94] calculations, respectively. The total length of the beamlines are $120 \mathrm{~m}$ and $290 \mathrm{~m}$, respectively. 
For the New $(g-2)$ Experiment the muons from pion decay will be mainly collected in the $290 \mathrm{~m}$ long AP2 beam line $(80 \%)$ and to a smaller extent in the Debuncher. The remainder of the beam line to the experiment serves to decay the pion content of the beam to reduce the hadronic flash. With $900 \mathrm{~m}$ total beamline, the initial pion flux is suppressed by a factor of 180 . The AP2 line has a large transverse acceptance (unnormalized $A_{x}=$ $35 \pi \mathrm{mm}-\mathrm{mrad}$ and $A_{y}=35 \pi \mathrm{mm}-\mathrm{mrad}$ ) and a momentum bite of $3 \%$. For transporting positive pions the field of all magnets has to be reversed and scaled by the momentum factor $3.11 / 8.89=0.350$ relative to antiproton collection. After the target station the beam is bent into a straight. FODO section extending for $170 \mathrm{~m}$, before it is bent left towards the injection into the left Debuncher arc at $290 \mathrm{~m}$. The spacing between FODO magnets is $13 \mathrm{~m}$. The bending angle leads to a negligible phase rotation of the muon spin, so that the forward produced muons maintain a high polarization of 0.98 . The long decay line allows the selection of the ideal pion over muon momentum ratio $\alpha=1.005$ and the integration of a larger $(2 \%)$ pion momentum bite. This results in a fourfold increase of collected muons per proton relative to $\mathrm{E} 821$. The length of the channel relative to $\mathrm{E} 821$ provides another factor of 2 gain.

\section{Pion production}

The E821 target was composed of 24 150-mm diameter nickel plates, 6.4-mm thick and separated by $1.6 \mathrm{~mm}$. The pion production yield for $24 \mathrm{GeV}$ protons was originally calculated with a simple Sanford-Wang parametrization of the cross sections and found in reasonable agreement with the observed rates. Here we use a pion yield per proton $Y_{\pi}$ into the E821 channel acceptance of $\approx 2.7 \times 10^{-5}$ within a $0.5 \%$ momentum band, based on observed rates and beam transport simulations.

First studies of particle yields into AP2 were performed using the MARS15 model [92]. The standard iconel target of the $\bar{p}$ source and the existing $1 \mathrm{~cm}$ radius Li lens were assumed, but with the polarity reversed and the current scaled by the factor 0.350 mentioned above, as the whole AP2 line is tuned to $3.11 \mathrm{GeV} / \mathrm{c}$. The results in Fig. 36 are promising and indicate an accepted pion yield per proton $Y_{\pi}=1.1 \times 10^{-5}$ per $0.5 \%$ pion momentum bin. This value is calculated at the end of the first deflection magnet PMAG in the target vault. This corresponds to a pion yield per $8 \mathrm{GeV}$ proton of 0.4 times the yield observed at $24 \mathrm{GeV}$, 
somewhat larger than estimated from naive beam energy scaling.

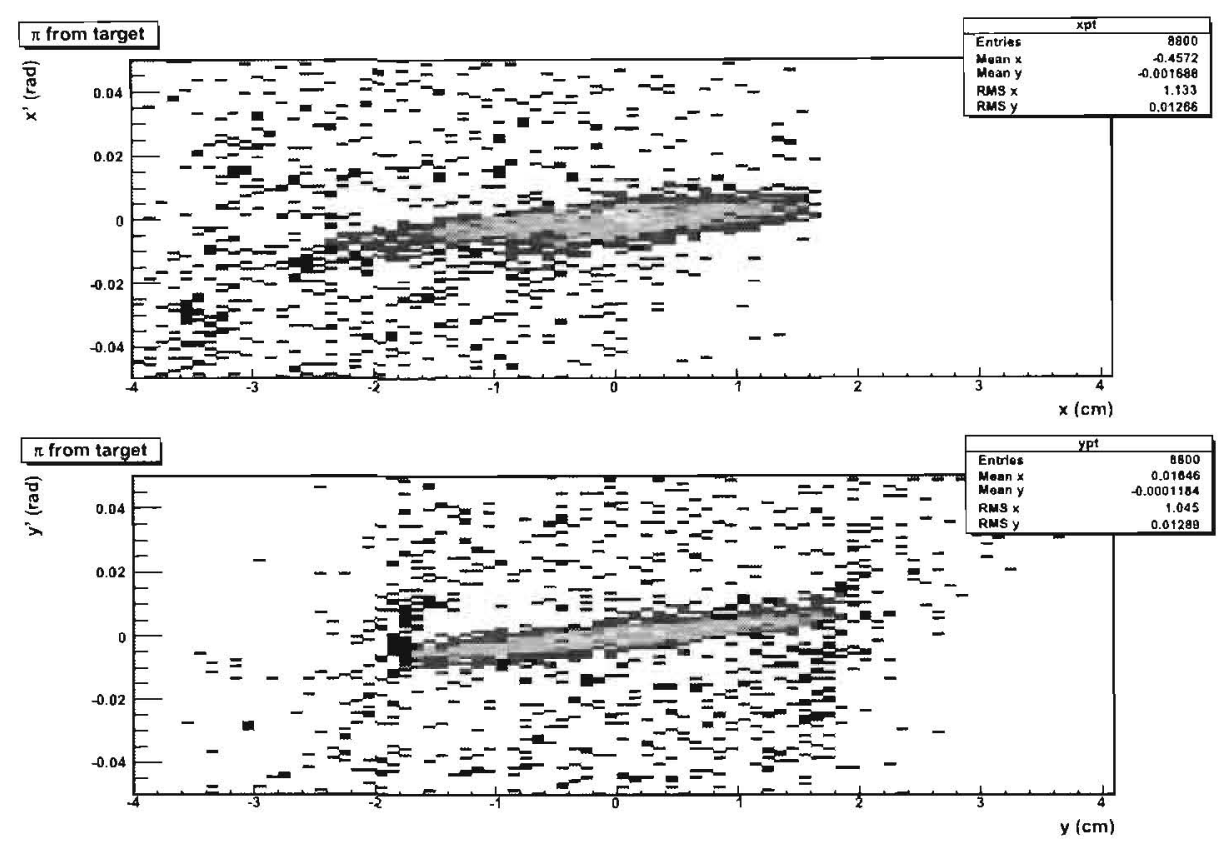

FIG. 36: MARS15 calculations of transverse pion phase space at the exit of the bending magnet PMAG at the antiproton target station.

\section{Transport in AP2}

The FODO lattice of AP2 is designed to transport the whole initial emittance collected by the Li lens. Thus increasing the lattice density of the FODO does not improve its acceptance for the primary pion beam. For the secondary beam of decay muons, however, the collection efficiency can be increased by reducing the size of the pion source, which leads to the acceptance of correspondingly larger decay angles. For a FODO lattice the maximum beta function $\beta_{\max }$ is proportional to the length $\ell$ of the FODO cell if the ratio of focal length $f$ to cell length $\ell$ is fixed. Thus if the length of the cell were reduced by a factor of $2, \beta_{\max }$ is reduced by a factor of 2 , and the beam size by a factor $\sqrt{2}$. Since this reduction occurs in both transverse planes, the increase in transmitted muon flux can be as large as a factor of 2. This scaling law was extensively studied in TURTLE simulations towards an improved E821 beamline and found to be roughly correct. The exact gain depends on the full figureof-merit and has to be calculated case by case. At present, we consider quadrupling the 
lattice density of the present AP2 beamline with cost effective permanent quadrupoles and elements of the existing E821 beamline. Pending further optimization studies, a reduction of the magnet spacing from presently $13 \mathrm{~m}$ to $3.25 \mathrm{~m}$ in the first part of the AP2 could increase the stored muon fraction by a factor of 1.8 compared to E821.

\section{Performance Estimate}

Fig. 37 sketches the basic figure of merit of the anticipated beamlines for the new FNAL

versus the previous BNL experiment. Particle numbers per $10^{12}$ protons ( $\mathrm{Tp}$ ) are presented as a function of the assumed beamline length. The pion curve indicates the number of pions entering the $(g-2)$ ring and generating the hadronic background flash. The muon curves denote the number of stored muons. The advantage of the FNAL set-up is evident. The significantly longer decay channel together with other beam transport improvements allows the collection of 6 times more muons at the experimental location. The number of pions per $\mathrm{Tp}$ is also reduced. The quantity relevant for the flash related background problem is the number of pions per fill, which is smaller by another factor of four due to the higher fill repetition rates at FNAL. 


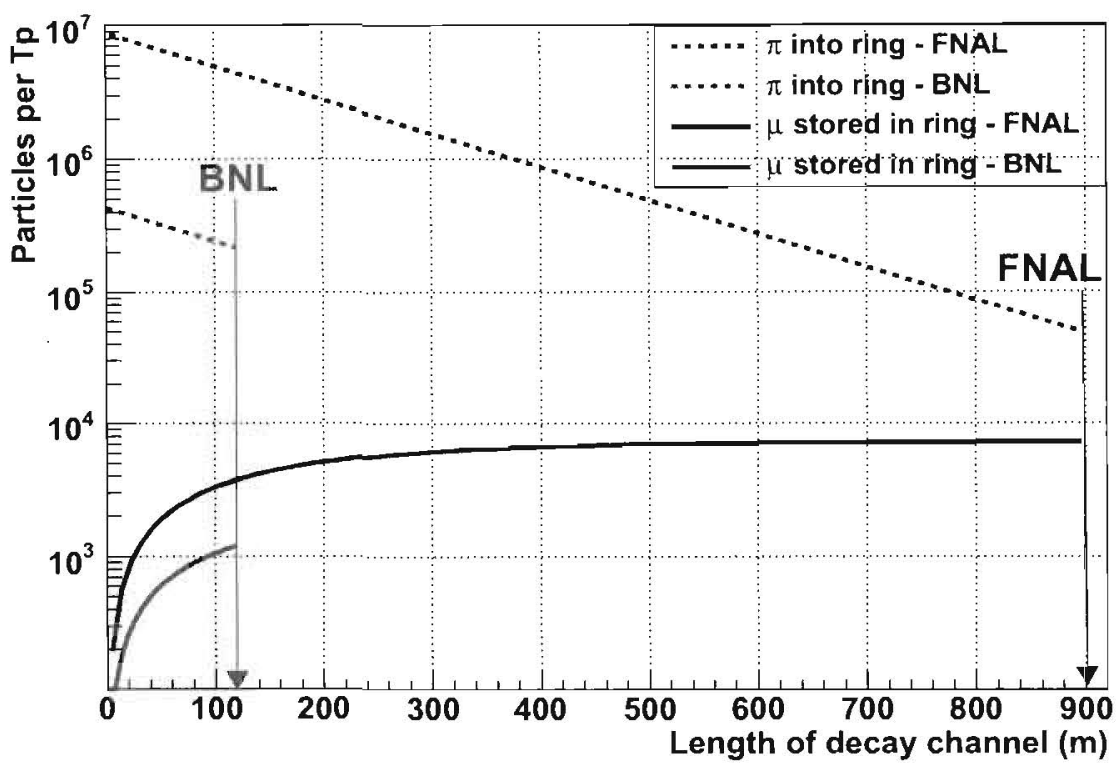

FIG. 37: Pions entering and muons stored in the $(g-2)$ ring for $10^{12}$ protons on target for the conditions of the previous BNL experiment and the proposed FNAL experiment. The horizontal axis shows the distance to the pion production target and the arrow indicate the locations of the experiments. In the New $(g-2)$ Experiment the dangerous pion background is significantly suppressed and the muon collection efficiency enhanced. 


\section{APPENDIX B: BEAM DYNAMICS AND SCRAPING}

\section{The Kicker and Quadrupoles}

The incoming bunched beam from the AGS is kicked on orbit by a fast muon kicker consisting of three identical pulse-forming networks and kicker magnets. [73] The fast muon kicker worked adequately during the running period of E821, but there are several maintenance items which must be attended to. The first kicker unit must be repaired to replace a cracked ceramic insulator, which prevented it from holding full voltage. We are also studying the absolute injection efficiency to see if an additional kicker module might improve the reliability and efficiency of injection. The rate calculations in this proposal do not assume any additional factor from the kicker.

The electrostatic quadrupoles, which provide the (weak) vertical focusing in the storage ring, worked well in E821. We do need to improve the lead geometry inside of the vacuum chamber to further reduce trapped electrons, as well as improve the lead configuration outside of the vacuum chamber where the high voltage feed-throughs are located to make them more reliable. These changes represent modest improvements and will not be discussed further in this proposal.

\section{Beam Dynamics in the Ring}

The storage ring is a weak focusing ring, with the field index $n<1.0$ determined by the strength of the electrostatic quadrupole field. In the limit of a continuous quadrupole field, the stored muons execute simple harmonic motion radially $(x)$ and vertically $(y)$ with the frequencies given by

$$
f_{y}=f_{C} \sqrt{n} \simeq 0.37 f_{C} ; \quad f_{x}=f_{C} \sqrt{1-n} \simeq 0.929 f_{C},
$$

where $f_{C}$ is the cyclotron frequency, and the field index $n$ is given by

$$
n=\frac{\kappa R_{0}}{\beta B_{0}} .
$$

The numerical values are for $n=0.137$. The frequencies in the ring are given in Table X.

One of the systematic errors which must be improved in the new experiment comes from muon losses out of the storage ring which result from processes other than muon 


\begin{tabular}{|l|l|l|l|}
\hline Quantity & Expression & Frequency & Period \\
\hline$f_{a}$ & $\frac{e}{2 \pi m c} a_{\mu} B$ & $0.23 \mathrm{MHz}$ & $4.37 \mu \mathrm{s}$ \\
\hline$f_{c}$ & $\frac{v}{2 \pi R_{0}}$ & $6.7 \mathrm{MHz}$ & $149 \mathrm{~ns}$ \\
\hline$f_{x}$ & $\sqrt{1-n} f_{c}$ & $6.23 \mathrm{MHz}$ & $160 \mathrm{~ns}$ \\
$f_{y}$ & $\sqrt{n} f_{c}$ & $2.48 \mathrm{MHz}$ & $402 \mathrm{~ns}$ \\
\hline$f_{\mathrm{CBO}}$ & $f_{c}-f_{x}$ & $0.477 \mathrm{MHz}$ & $2.10 \mu \mathrm{s}$ \\
$f_{\mathrm{VW}}$ & $f_{c}-2 f_{y}$ & $1.74 \mathrm{MHz}$ & $0.574 \mu \mathrm{s}$ \\
\hline
\end{tabular}

TABLE X: Frequencies in the $(g-2)$ ring. $C B O=$ coherent betatron oscillation; $V W=$ vertical waist; $a, c$ refer to spin precession $\omega_{a}$ and cyclotron frequencies respectively.

decay. In E821 we reduced these losses by scraping off particles on the edge of the storage volume. Scraping is defined as the creation of a gap of several $\mathrm{mm}$ between the beam and the collimators that will either eliminate altogether or drastically reduce particle losses during data collection time. This was achieved by asymmetrically powering the electrostatic quadrupoles during and after injection for 10-15 $\mu$ s and scraping the beam on collimators placed around the ring. This asymmetry caused the beam to be lifted and moved sideways during this scraping time. At the end of the scraping period, the beam was returned to the equilibrium orbit with a $5 \mu$ s time constant. While losses were reduced from $0.6 \%$ per muon lifetime in the ring with no scraping to $0.2 \%$ with scraping, we will need to do better in the new experiment.

Because of the small inflector size relative to the storage volume, shown in Fig. 38, the phase space in the ring is not uniformly filled. This causes the bunched beam to oscillate coherently both vertically and horizontally in the storage ring. For a detector at a fixed point in azimuth, the apparent radial motion of the beam is the difference frequency between the cyclotron frequency and the horizontal betatron frequency given in Eq. B1. The inflector image is re-formed every betatron wavelength, so that this "waist" in the beam also moves around the ring with the difference frequency between the the cyclotron frequency and twice the radial (vertical) betatron frequency. Since the detector acceptance depends on the radial position of the muon when it decays, the coherent radial betatron oscillations (CBO) amplitude-modulate the time spectrum. The modulation effect decreases in the time due to 


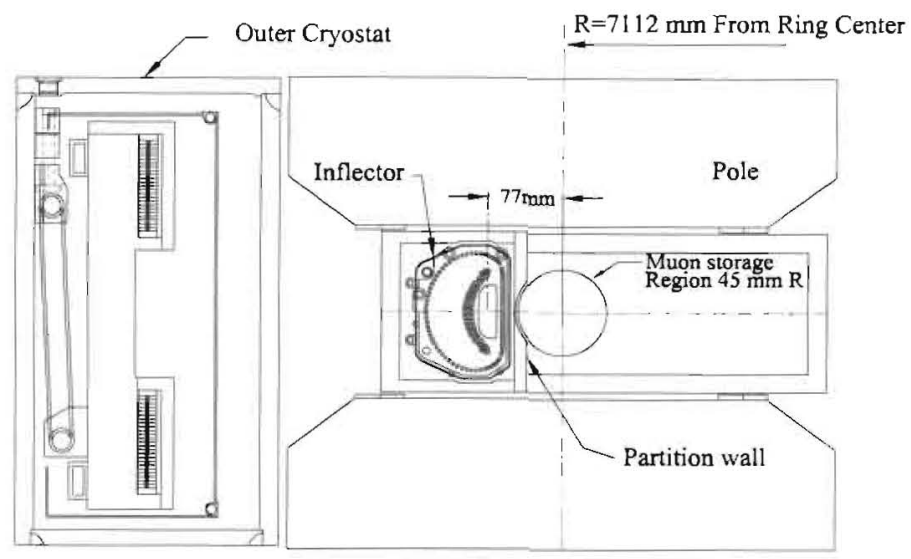

(a)

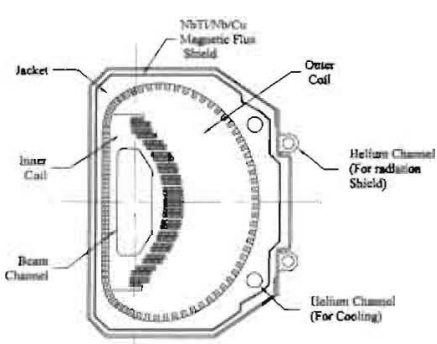

(b)

FIG. 38: (a) The geometry of the inflector exit and the muon storage volume. (b) The cross section of the inflector.

the "natural" chromaticity of the betatron oscillations, which slightly mix up phases of the particle oscillations. In E821 we measured a decoherence time of about $100 \mu$ s for the CBO, and the muon lifetime was $64.4 \mu \mathrm{s}$.

In the new experiment we wish to reduce the CBO effects, and to improve the scraping of the beam. Two approaches to reduce the coherent betatron motion and scrape the beam have been proposed:

1. Using a RF dipole field during the time immediately after injection to first drive the coherent betatron oscillations to scrape the beam, and then to reverse the phase to damp the CBO. This technique would get rid of the main CBO but not the "waist" motion in the beam.

2. A scheme which causes a fast phase mixing in the betatron tune through the introduction into the machine lattice of a nonlinear focusing element such as an octupole. Preliminary studies indicate that the CBO modulation can be minimized by applying this field during less than a hundred turns after injection. This will also serve the purpose of scraping the beam simultaneously with the CBO decoherence. 


\section{Oscillating Dipole Method of Scraping}

In E821 we have estimated the horizontal CBO amplitude at injection both directly and indirectly [96]. The first method involved the fiber beam monitors (see Fig. 39) which consist of eight $0.5 \mathrm{~mm}$ diameter scintillating fibers which are inserted into the beam to measure the profile. The signal from a single vertical and single horizontal fiber are shown in Fig. 40 where the beam motion across the fiber is clearly seen. The measured CBO amplitude was found to $\simeq 7 \mathrm{~mm}$ with a frequency $\omega_{C B O}=\omega_{C}(1-\sqrt{1-n}) \simeq 470 \mathrm{KHz}$.

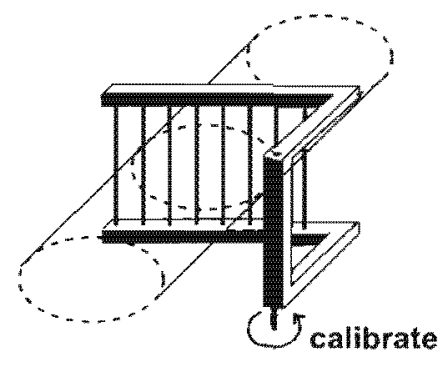

$\mathrm{x}$ monitor

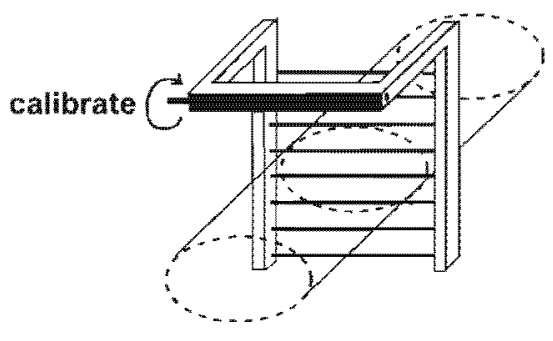

y monitor

FIG. 39: A sketch of the $x$ and $y$ fiber beam monitors. The fibers are $0.5 \mathrm{~mm}$ in diameter. For calibration, the entire "harp" rotates into the beam so that all fibers see the same beam intensity.

This beam CBO manifested itself, among other ways, as a modulation of the number of detected positron by the electromagnetic calorimeters with an amplitude of $\simeq 1 \%$. Monte Carlo studies showed that an amplitude of $\simeq 7 \mathrm{~mm}$ for the CBO would cause that modulation amplitude. Vertically the CBO amplitude is much smaller but still visible from the data at early times. With similar beam injection conditions the CBO has a well defined frequency, amplitude and phase. We propose to use this fact to both scrape the beam and eliminate the horizontal and vertical CBO of the beam due to the motion of the beam center.

This proposal uses a set of four plates, with ell $=1 \mathrm{~m}$ long azimuthally and placed in the configuration of the quadrupole plates in the g-2 ring. We will then apply a voltage difference between the opposite plates with a frequency equal to the horizontal (horizontal plates) and vertical (vertical plates) CBO. For scraping the beam we will apply the voltage in phase with the beam CBO phase to increase the CBO amplitude. To eliminate the CBO the phase will be opposite. To estimate the voltage needed we will consider here the horizontal $\mathrm{CBO}$, but the same method can be applied for the vertical $\mathrm{CBO}$. The functional form of the 

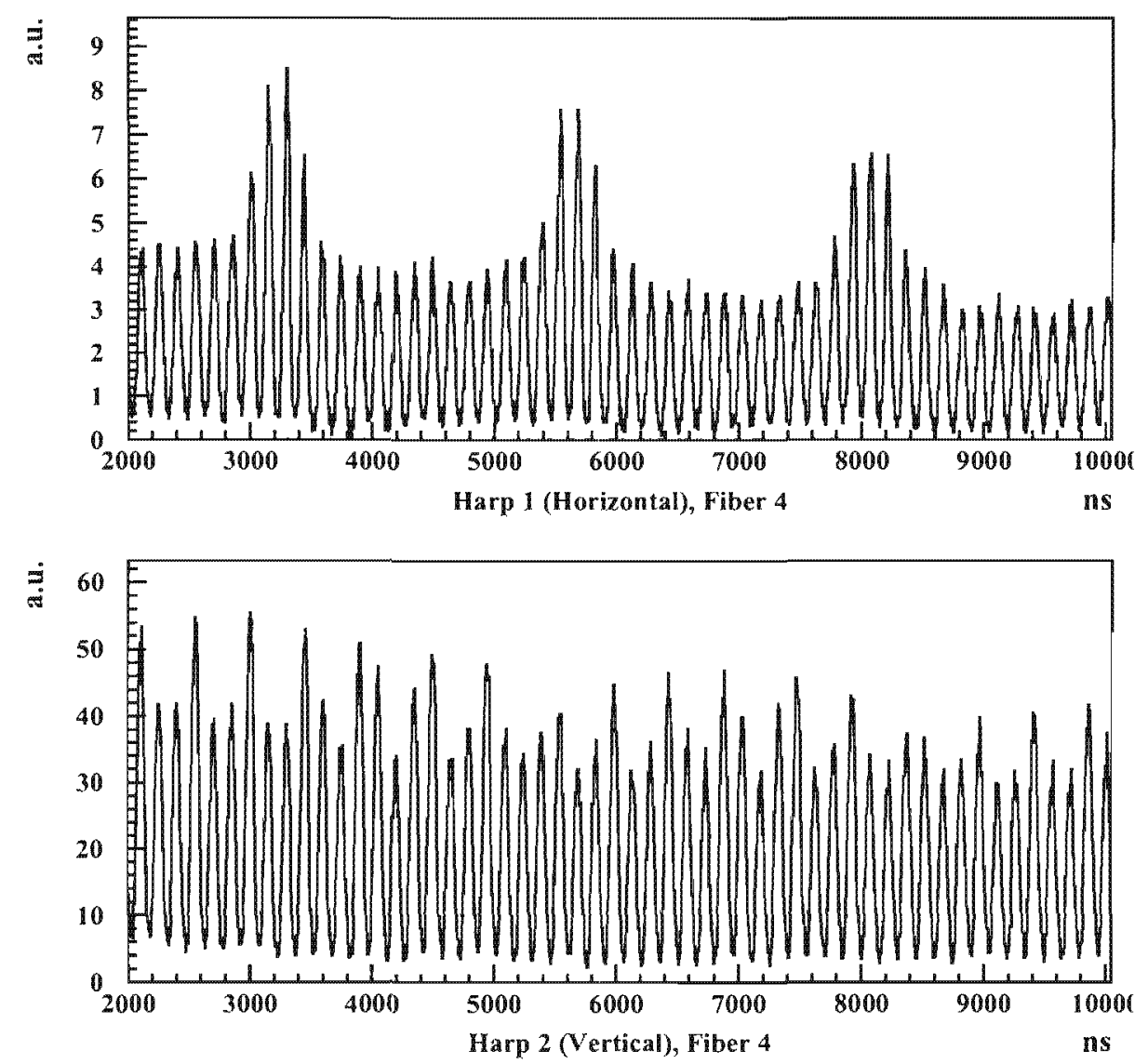

FIG. 40: The phototube output from a single $x$ and $y$ fiber. The CBO frequency (horizontal) and VW frequency (vertical) are clearly seen (see Table X).

horizontal dipole electric field, $E_{x}(t)$, is

$$
E_{x}(t)=E_{x 0} f(s) \cos \left(\omega_{C B O} t+\theta_{0}\right)
$$

where $f(s)=1$ for the space between the plates and 0 outside them. We take as $t=0$ the time the muon beams enter the electric plates for the first time. Then the equation of motion can be written as

$$
\ddot{x}+\omega_{C}(1-n) x=\omega_{C}^{2} R\left(\frac{e E_{x 0}}{\beta B}\right) f(t) \cos \left(\omega_{C}\left(1-\sqrt{1-n} t+\theta_{0}\right)\right)
$$

where $\beta \equiv v / c$ and

$$
f(t)=\left\{\begin{array}{l}
1, T q<t<\left(T q+\frac{1}{\nu}\right), q=0,1, \cdots N \\
0, \text { otherwise }
\end{array}\right.
$$


Where $T \simeq 149 \mathrm{~ns}$, the cyclotron period of the ring. The exact solution of equation 3 with $f(t)$ given by Eq. B5 is

$$
\begin{aligned}
& x=a(t) e^{i \omega_{x} t}+a^{*}(t) e^{-i \omega_{x} t} \\
& a=a_{0}-i e^{i \theta_{0}} \frac{N+1}{4 \sqrt{1-n}}\left(\frac{E_{x 0} l}{\beta B}\right)\left[1+\frac{e^{i 2 \theta_{0}}\left[1-e^{-i 2 \omega_{C}(1-\sqrt{1-n} T(N+1))}\right]}{N+1\left[1-e^{-i 2 \omega_{C}(1-\sqrt{1-n}) T}\right]}\right] .
\end{aligned}
$$

where $a_{0}=\left(x_{\max } / 2\right) e^{i \alpha}$ corresponds to $t=0$ and defines the electric field phase $\theta_{0}$. After $N+1$ turns we get Eq. B7 which for large $N>10$ simplifies to

$$
a=a_{0}-i e^{i \theta_{0}} \frac{N+1}{4 \sqrt{1-n}}\left(\frac{E_{x 0} l}{\beta B}\right),
$$

where $B$ is the storage ring dipole magnetic field. For $x_{\max }=0.7 \mathrm{~mm}, N=60$ (i.e. about $10 \mu \mathrm{s}), n=0.142$, and $B=1.45 \mathrm{~T}$ we need $E_{x 0} \simeq 0.9 \mathrm{kV} / \mathrm{cm}$ at the CBO frequency of $\simeq 470 \mathrm{KHz}$. For a plate separation of $10 \mathrm{~cm}$ it means a voltage amplitude of $\simeq 9 \mathrm{KV}$ across the opposite plates horizontally driven at $470 \mathrm{KHz}$, which is quite reasonable.

The expected beam losses after scraping the beam are going to be dominated by the vertical scraping since it is expected that horizontally we wouldn't need to scrape more than $\simeq 7 \mathrm{~mm}$ oscillation of the beam at injection. Horizontally we would therefore just wait of the order of $5 \mu \mathrm{s}$ for the beam to scrape off the collimators after which we would apply the voltage estimated above to eliminate it. Vertically we would need to apply a voltage for about $5 \mu$ s after injection in phase with the natural one so that we induce an overall vertical oscillation of the order of $5 \mathrm{~mm}$ after which we will flip the sign of the phase to eliminate it. The total beam losses induced by this method of scraping for a beam gap of $5 \mathrm{~mm}$ vertically and $7 \mathrm{~mm}$ horizontally are estimated to be less than $20 \%$.

\section{Pulsed Octupole Method to Remove the CBO}

This method effects a fast phase mixing by an introduction of a nonlinear focusing element in the machine lattice[97]. The nonlinearity induces a dependence of betatron tunes on amplitudes of transverse oscillations $\left(\frac{\delta Q}{\delta a^{2}}\right) \cdot[98]$ The CBO modulation could be minimized during a few tens of turns in the storage ring. Using a time (up to 100 turns) to apply a pulsed closed orbit distortion can make the $\mathrm{CBO}$ vanish while simultaneously scraping the beam. 
The rectangular symmetry of the vacuum chamber permits one to install an octupole coil inside the vacuum chamber. The coil can be wound around an area of $6 \mathrm{~cm}$ radius as shown in Fig. 41, while the beam has $r=4.5 \mathrm{~cm}$. In a preliminary design, a coil length of $2 \mathrm{~m}$ was chosen, located in a section free from electrostatic focusing and kickers. The field lines have been calculated by the computer code MERMAID. [99]

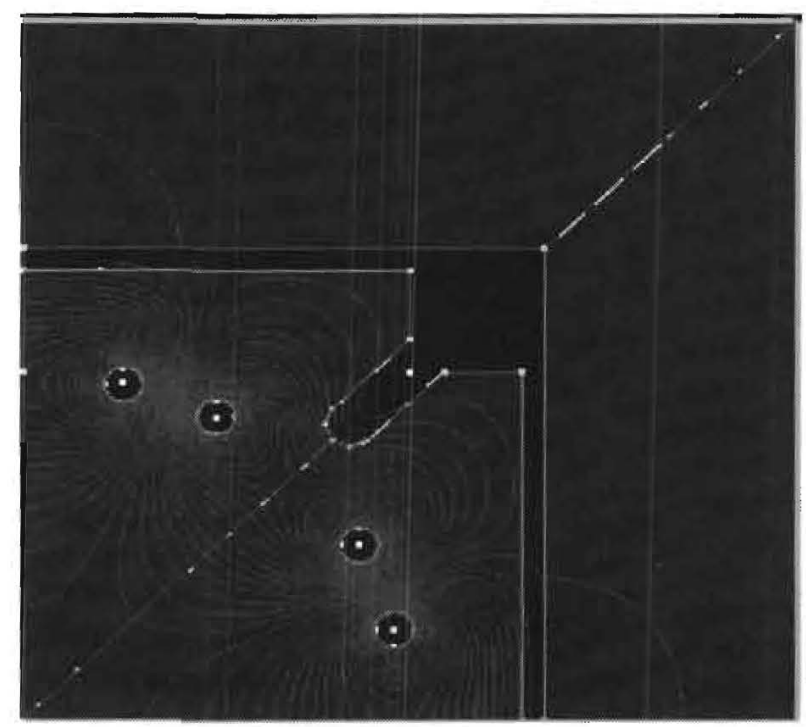

FIG. 41: Octupole coil in vacuum chamber and magnetic field lines

Particle tracking was done with electrostatic focusing of the $(g-2)$ ring for different octupole field strengths, and for several residual horizontal angles after the kicker. The RING code was used for the tracking,[100] for 10000 particles. The initial phase-space distributions were assumed to be uniform in both the vertical and radial directions. The muons were tracked for 100 turns. The initial and stored phase space distributions are shown in Figs. 42.

During tracking, the amplitude of the CBO was evaluated after each turn by the expression:

$$
A=\sqrt{\frac{\left(1+\alpha_{x}^{2}\right)\langle x\rangle^{2}+2 \alpha_{x} \beta_{x}\langle x\rangle\langle x \prime\rangle+\beta_{x}^{2}\langle x\rangle^{2}}{\beta_{x}}+\frac{\left(1+\alpha_{y}^{2}\right)\langle y\rangle^{2}+2 \alpha_{y} \beta_{y}\langle y\rangle\langle y \prime\rangle+\beta_{y}^{2}\left\langle y^{\prime}\right\rangle^{2}}{\beta_{y}}}
$$

where $\langle x\rangle\langle x \prime\rangle,\langle x\rangle^{2},\langle x\rangle\langle x \prime\rangle,\langle x\rangle^{2}$ are average over the ensemble coordinates and angles and their squared values. $\alpha$ and $\beta$-. are corresponding Twiss parameters.

Fig. 43 shows the $\mathrm{CBO}$ amplitude versus turn number $\mathrm{N}$, for different octupole strengths. 

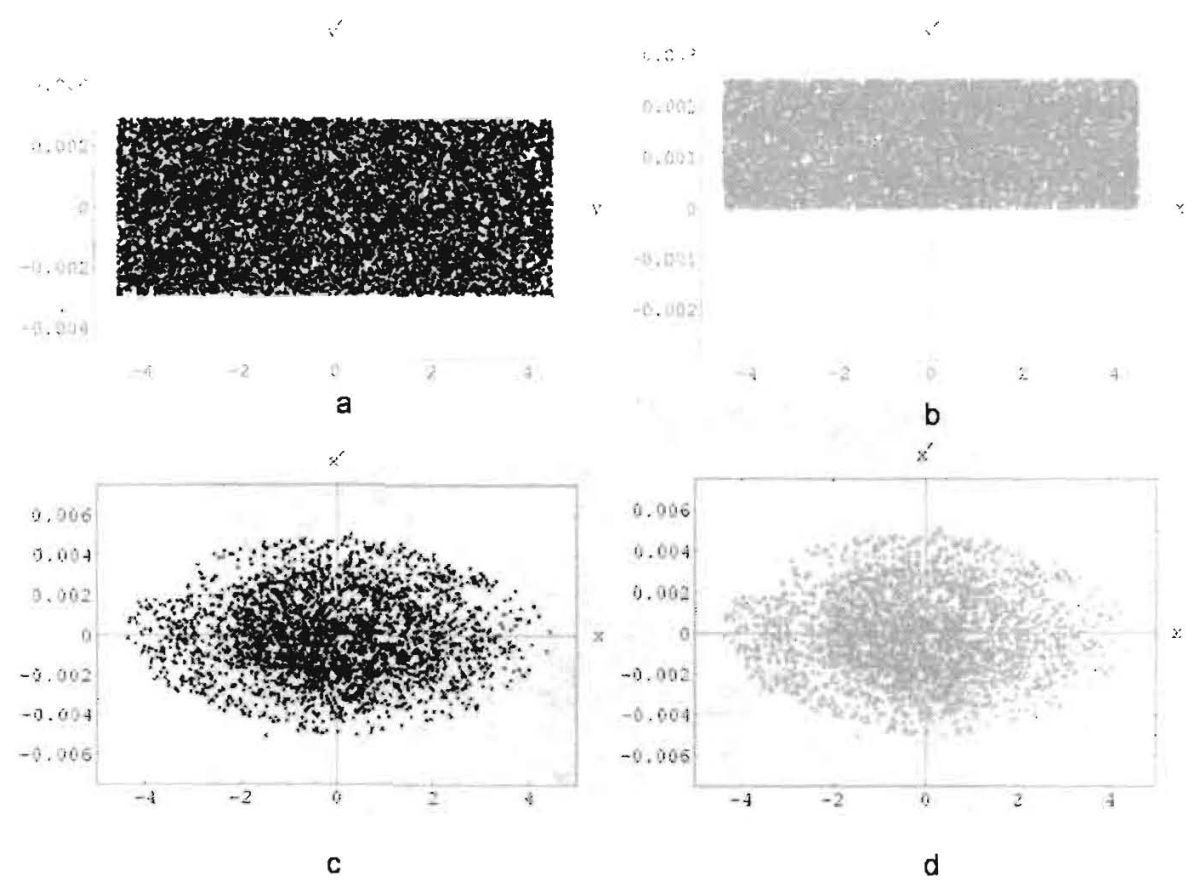

FIG. 42: Initial and final phase distributions. (a) Initial vertical distribution. (b) Initial radial distribution. (c) Stored radial distribution. (d) Stored radial distribution.

One can see an octupole gradient of $0.8 \mathrm{G} / \mathrm{cm}^{2}$ greatly reduces $\mathrm{CBO}$ amplitude by the 30-th turn. The tracking shows that neither this reduction factor, nor the amplitude beating after the octupole is removed, depend on the residual angle. About $50 \%$ of the beam is lost using this method.

Calculations show that to provide the optimal octupole gradient of $0.8 \mathrm{G} / \mathrm{cm}^{2}$, a pulse current of $2.5 \mathrm{kA}$ is needed. This will need to be a pulsed octupole, since the octupole field, as well as any magnetic fields induced by eddy currents in the vacuum chamber or other conductors nearby, must be negligible before data collection can begin. Simulations used both a square (ideal) current pulse and a sinusoidal one, and little difference was found compared to the rectangular one. The parameters of an LCR pulse generator are, Voltage $\mathrm{V}=1.3 \mathrm{kV}$, Capacitance $\mathrm{C} \approx 1 \mu \mathrm{F}$, Period $\mathrm{T} \approx 10 \mu \mathrm{sec}$. The octupole coil can be made from water-cooled copper pipe of $1 \mathrm{~cm}$ in diameter. The energy dissipated in such a coil per pulse is about $1 \mathrm{~J}$.

An alternative electrostatic octupole is able to do the same, but its realization looks from practical point of view much more difficult, because of the symmetry imposed by the trolley 


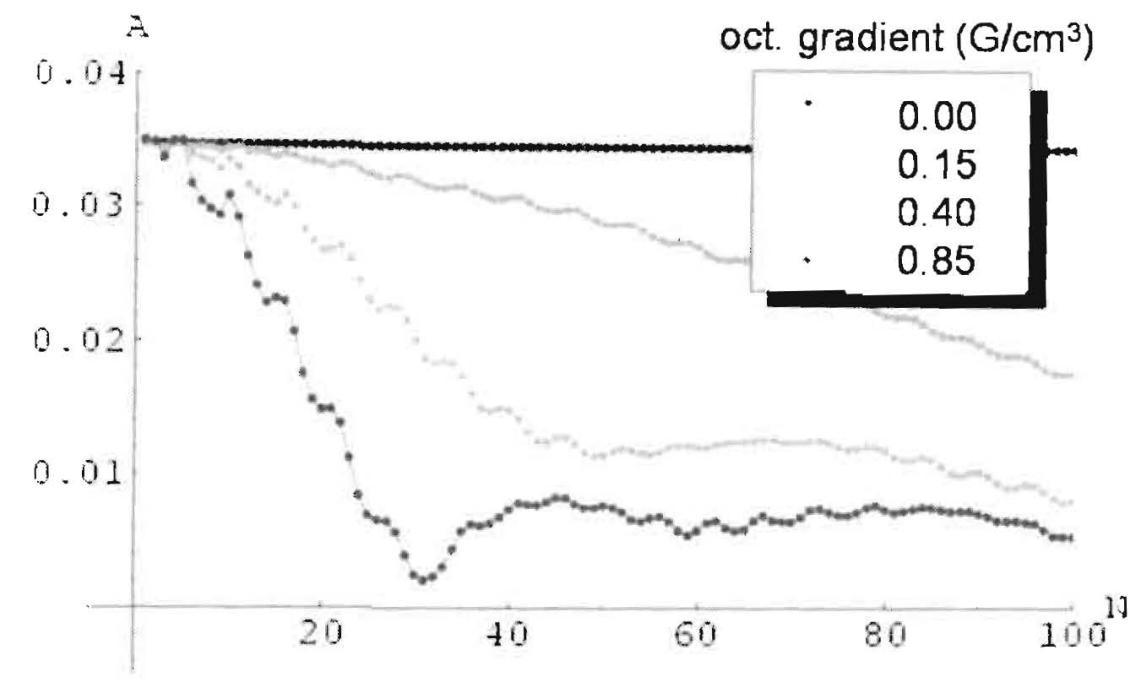

FIG. 43: Behavior of the CBO amplitude as a function of turn number and octupole strength.

rails which go all the way around inside the vacuum chamber with four-fold symmetry. 


\section{APPENDIX C: NEW CALORIMETERS}

The basic material design requirements for a new calorimeter are largely unchanged; it must be dense and fast. Additionally, the new calorimeter must be segmented transversely with respect to the incoming positron, so that simultaneous events can be distinguished 4 out of 5 times. Building new $\mathrm{Pb} / \mathrm{SciFi}$ calorimeters with fibers running longitudinally (i.e., rotated by 90 degrees) is one possible solution. The downstream face of the detector would be a fiber / lead grid that could be readout in small and independent segments. Members of our Collaboration have built detectors of this type in the past, with incoming photons nearly co-linear with the fiber direction. This re-oriented $\mathrm{Pb} / \mathrm{SciFi}$ option is attractive in principle, but there are two drawbacks. First, the downstream space is limited by the existing vacuum chamber structures. A denser detector is desired to open up additional space for the readout system. Second, simple simulations using $\rho_{M}=2.5 \mathrm{~cm}$ and the requirement that showers be separated by at least $2 \rho_{M}$, indicate a pileup separation factor of no better than 3 (the simulation uses the actual distribution of positrons on the calorimeter face, see Fig. 44). The goal of the new detectors is to separate simultaneous showers by a factor of about 5 . To do so requires the detector to have a smaller Moliere radius.

We have designed and built a simple detector made of alternating layers of $0.5-\mathrm{mm}$ thick tungsten (W) plate and 0.5-mm-diameter plastic scintillator fiber ribbons. A NIM paper is in press at the time of this writing [15]. We briefly summarize some of the interesting findings from our studies.

The prototype module is $4 \times 6 \times 17 \mathrm{~cm}^{3}$. The calculated radiation length is $0.69 \mathrm{~cm}$ and the Moliere radius is $1.7 \mathrm{~cm}$. Both are much smaller than the $\mathrm{Pb} / \mathrm{SciFi}$ design used in E821. The fiber ribbons are oriented vertically so that the positrons, which are curling inward, must cross $W$ layers and initiate showers. The full sized detector will be made as one monolithic structure with dimension $15 \mathrm{~cm}$ high by 21 -cm radial. The $11-\mathrm{cm}$ depth represents more than $15 X_{0}$. Fibers will be either gathered into small bundles on the downstream side forming individual "segments" or a direct array of lightguide couplers will be attached to provide the segmentation. At the present time, we are exploring either a $5 \times 7$ array of $3 \times 3 \mathrm{~cm}^{2}$ segments or, with slight overall dimension changes to the SciFi block, a coarser array of $4 \times 5$ segments with front faces of $4 \times 4 \mathrm{~cm}^{2}$. Either segmentation will easily meet our design specifications related to pileup reduction. 

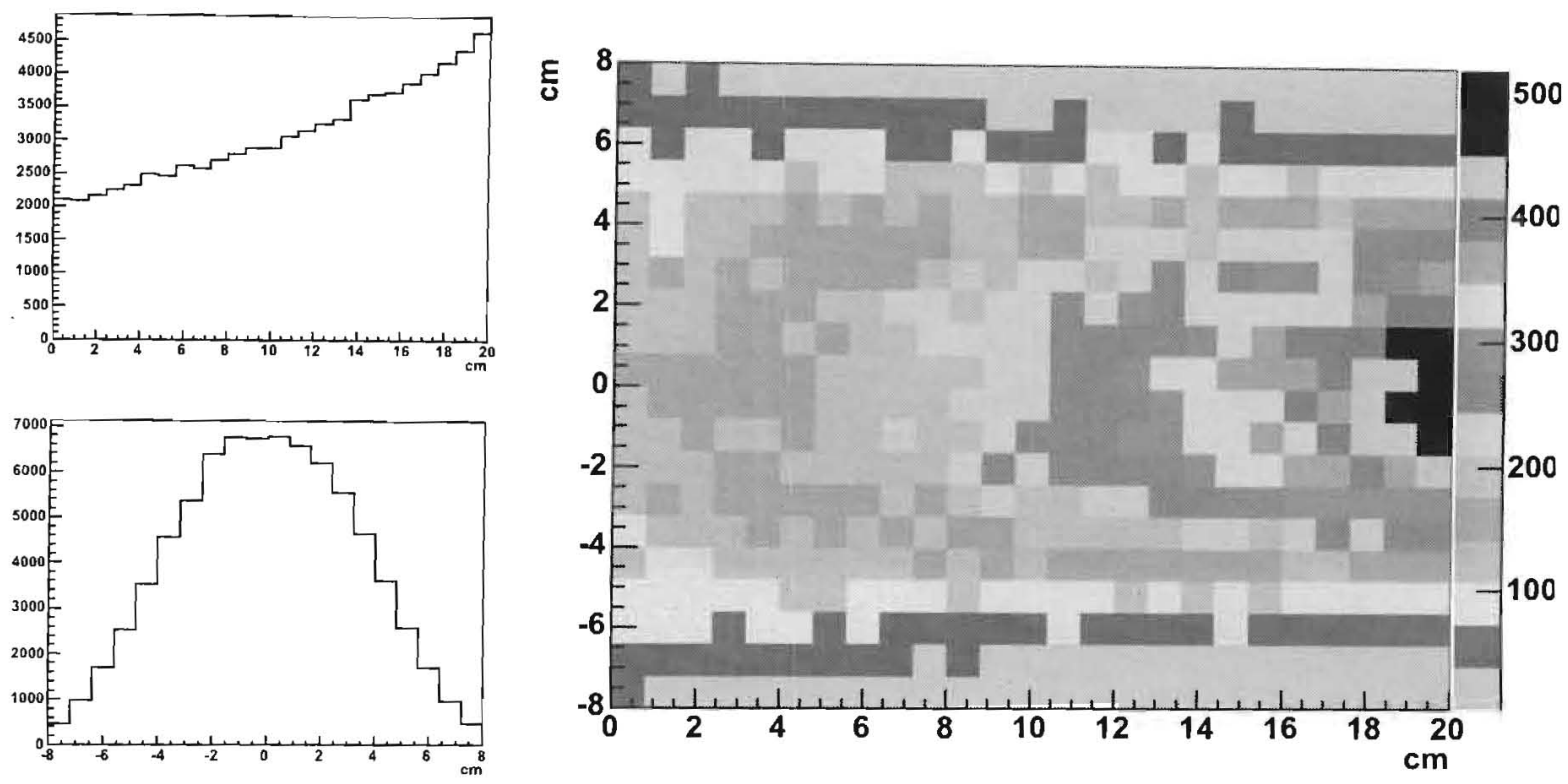

FIG. 44: Right panel: Hit position of positrons on calorimeter front face from from GEANT simulation. Top left panel: radial distribution; storage ring edge is at large $x$ values. Bottom left: vertical distribution.

The prototype is shown in Fig. 45a. The full conceptual design array of $4 \times 4 \times 11 \mathrm{~cm}^{3}$ modules is shown in Fig. 45b; 24 such arrays are required for the proposed muon $(g-2)$ experiment.

We have developed a simple and effective assembly procedure for these modules that will assure consistent uniformity of the completed modules. Quotes $[101,102]$ have been obtained for the fiber ribbons and flat tungsten plates, required for the full production of 24 calorimeter modules. The total cost of the calorimeter modules just under $\$ 0.5 \mathrm{M}$, with construction by the technicians at Illinois.

The prototype used 0.5-mm diameter BCF-20, "green-emitting" scintillating fibers obtained from Saint-Gobain Crystals [101]. These fibers were conveniently available owing to a large production for an independent project. They arrived as $12-\mathrm{cm}$ wide by $17.5-\mathrm{cm}$ long "ribbons." Each ribbon came as a self-contained structure with the individual 0.5-mm fibers held adjacent by a coating of a polyurethane-acrylic blend cement. We split each ribbon into two $6-\mathrm{cm}$ wide strips to match the tungsten plate widths. The fibers are coated with a $10-15 \mu \mathrm{m}$ thick white extra mural absorber for better light transmission. For the final 


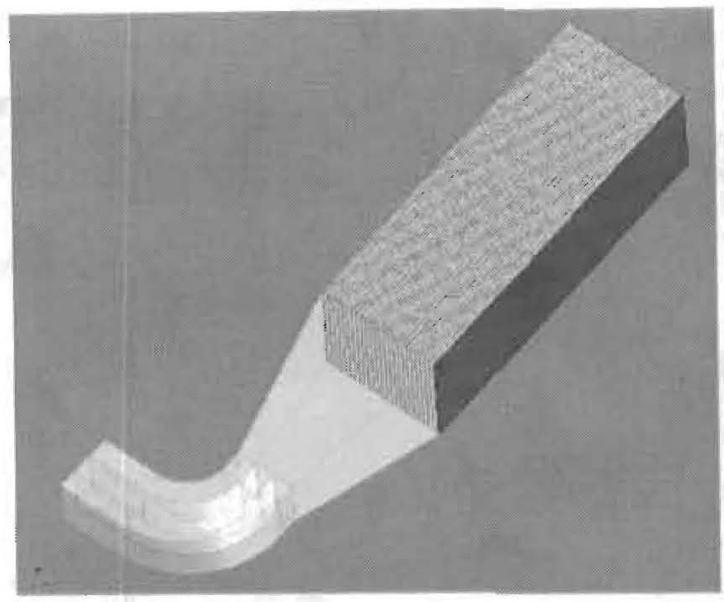

(a)Prototype module and guide

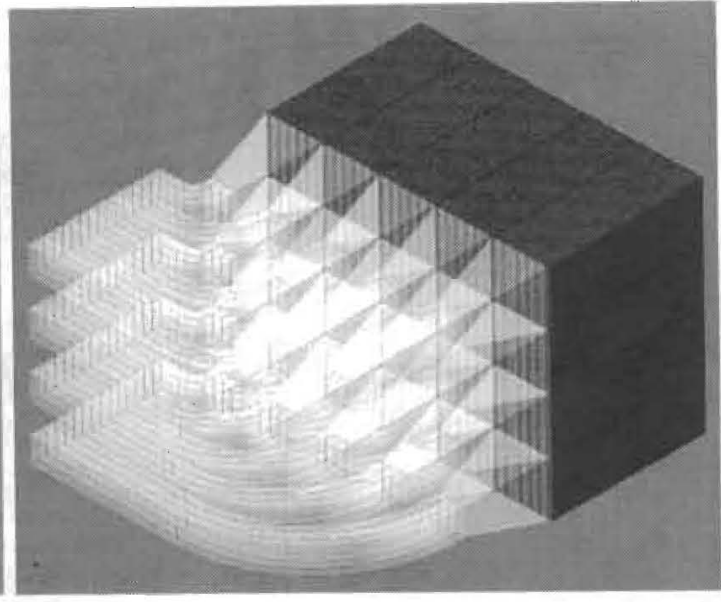

(b)Proposed array of 20 modules

FIG. 45: a) Prototype $4 \times 6 \times 17 \mathrm{~cm}^{3}$ module and b) proposed array of twenty $4 \times 4 \times 11 \mathrm{~cm}^{3}$ modules. The lightguides must curl toward the $(g-2)$ storage ring center and connect to PMTs outside of the storage ring magnetic field.

production modules, BCF-10 (or equivalent) "blue" fibers will be used to better match the quantum efficiency of the readout device and for faster time response.

The W/SciFi detector was first tested at the Paul Scherrer Institut (PSI) and at the Meson Test Beam at Fermilab. The focus of the test beam measurements was on calorimeter linearity and energy resolution. While neither beamline was optimized to provide a small momentum resolution or spot size, sufficient performance information was obtained to compare measurement to GEANT-4 based Monte Carlo simulations.

The typical $(g-2)$ energy threshold for including events is $1.8-2 \mathrm{GeV}$; positrons are only accepted above this threshold. A resolution of $\sim 10 \%$ (defined as $\sigma / E$ for a simple Gaussian fit) would give an acceptable performance for this threshold in a future $(g-2)$ experiment. The response of the prototype tungsten detector is shown in Fig. 46 for $2 \mathrm{GeV}$ electrons impacting at 5 degrees with respect to the fiber axis. An entrance cut is made using beamline wire chamber information to select the central $15 \mathrm{~mm}$ by $30 \mathrm{~mm}$ (width by height) of the 40 - by $60-\mathrm{mm}$ detector face. Even with no corrections for leakage into side detectors, or adjustments for sub-optimal light collection, or beam momentum uncertainty, the resolution at $10.1 \pm 0.3 \%$ meets the experimental goal.

Our goal is to understand the intrinsic sampling resolution of this detector and compare it to simulation. While the stochastic term is mainly determined by the sampling fluctuations 
intrinsic to the active-to-absorber material ratio and the effective layer thickness, additional contributions enter from photo-statistics. A 5\% contribution exists from photo-statistics, because the measured photoelectron (pe) yield is $400 \mathrm{pe} / \mathrm{GeV}$. This is a smaller light yield than we would expect had the lightguide been better matched in area to the photomultiplier tube and if blue fibers were used instead of green (higher quantum efficiency). Two factors that scale with energy contribute to energy degradation - the transverse leakage, and the momentum uncertainty of the test beam. The leakage can be explored with data cuts and simulation; the $\triangle P / P$ is unknown but estimated to be a few percent. In Fig. 47, we plot the FNAL data fit to

$$
\frac{\sigma(E)}{E}=\sqrt{\frac{A^{2}+\Delta_{p e}^{2}}{E}+B^{2}}
$$

Here $A / \sqrt{E}$ represents the intrinsic sampling term, $\Delta_{p e} / \sqrt{E}$ is the photo-statistics uncertainty, and $B$ is a linear term. The term $\Delta_{p e}$ is fixed at $5 \%$ and $E$ is given in GeV. The upper curve is a fit based on data where a $25 \mathrm{~mm}$ "wide cut" in the entrance width of the beam was used, while the lower curve is a fit based on a $5 \mathrm{~mm}$ "narrow cut." The change affects both the sampling and the constant term as they are not easily separable, given the statistics. The narrow cut result minimizes, but does not eliminate, the leakage, resulting in $A_{\text {meas }}=11.8 \pm 1.1 \%$ and $B_{\text {meas }}=3.7 \pm 1.3 \%$ for the stochastic and constant terms, respectively.

The sampling fluctuation component can be predicted using a complete GEANT-4 model. A plot of this resolution versus energy for simulated electrons impacting on the module center at a 5-degree angle is shown in Fig. 48. Three curves are presented representing a high-statistics "pencil beam" with a $1 \mathrm{~mm}$ spot size in both dimensions, as well a separate simulation with data-like cuts of 5 - and 25 -mm entrance widths, which match the narrow and wide definitions for the data. The most appropriate comparison to data is the narrow cut, which yields $A_{\text {sim }}=10.6 \pm 0.8 \%$ and $B_{\text {sim }}=2.9 \pm 1.1 \%$ for the stochastic and constant terms, respectively. The $B$ term is representative of the leakage, since no $\Delta P / P$ uncertainty contributes for Monte Carlo. If we deconvolute the leakage contribution from the $B$ term in the data, a $\Delta P / P$ of $\approx 2.3 \%$ is implied, which is not unreasonable.

The simulation is, not surprisingly, somewhat better than the actual prototype-- $A_{\text {sim }}=$ $10.6 \pm 0.8 \%$ vs. $A_{\text {meas }}=11.8 \pm 1.1 \%$. Detector construction imperfections can contribute, as would non-uniform light collection in the guide. However, to explore this comparison more 


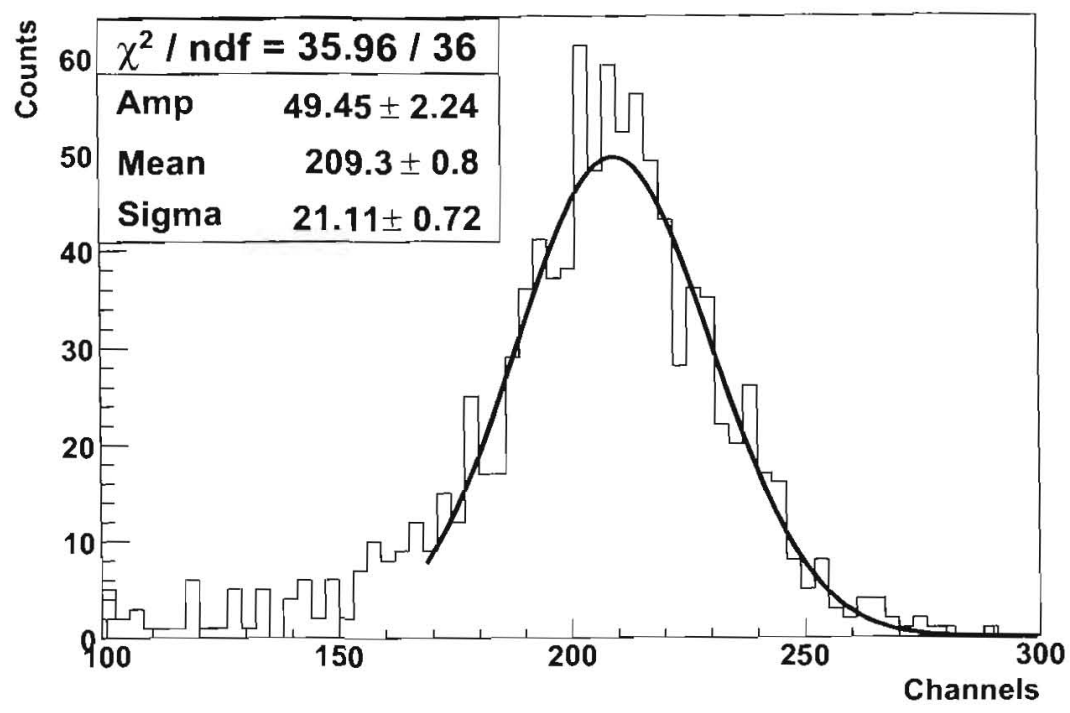

FIG. 46: Example raw W/SciFi detector ADC data for a $2 \mathrm{GeV}$ electron beam impacting at a 5 degree incidence. A modest containment cut of $15 \mathrm{~mm}$ width is made.

completely will require a larger test module with improved readout and a better controlled test beam environment. Note, that we carefully checked the GEANT-4 cut parameters, but found no dependence on them that altered our results.

Based on these studies, we intend to build a larger prototype that allows for full shower containment and readout by multiple optical devices. We are considering two options. The conservative plan is to use 29- $\mathrm{mm}$ (1-1/8 in) PMTs whose basic characteristics are understood and standard. We are using an array of 340 Photonis XP-2982, 11-stage PMTs for the muon lifetime experiment MuLan at PSI. The similar 10-stage XP-2972 is more appropriate for the New $(g-2)$ Experiment because of the high light yield from the calorimeter modules. Both tubes were carefully evaluated by us and feature similar important characteristics: low noise, high gain, no detectable after-pulses. We will have to design a robust, rate-dependent base as the initial rate of up to a few $\mathrm{MHz}$ is higher than what can be comfortably handled by the stock resistor divider network in the simplest Photonis bases. For E821, we developed a. gated base that allowed the PMT to be "turned off" during injection and turned back on within $1 \mu$ s after injection. Depending on the level of hadronic flash, we may need to incorporate this gating feature--therefore, we will plan to do so in our design.

The rapid development of silicon photomultiplier tubes (SiPMs) and their packaging 


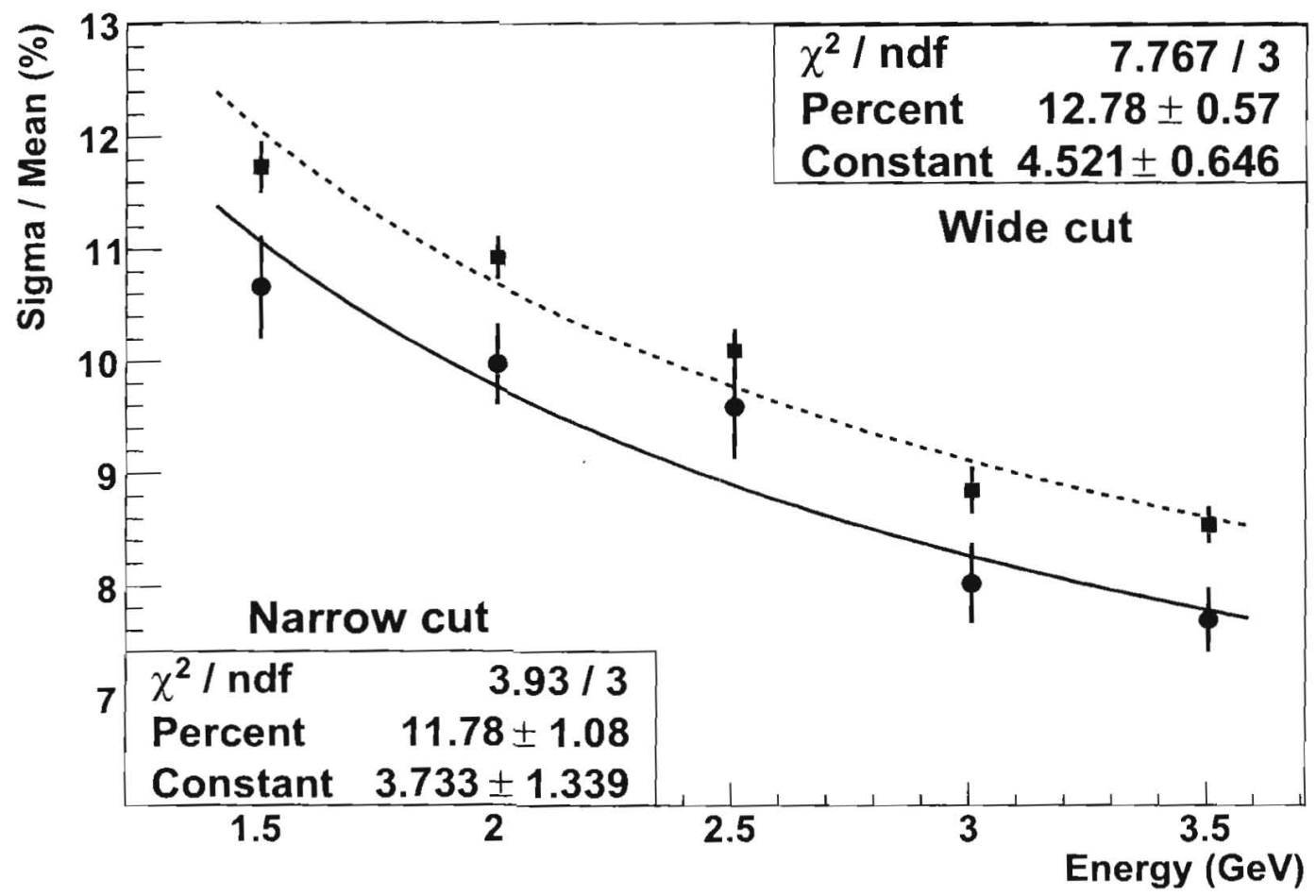

FIG. 47: Measured resolution at 5-degree impact angle versus energy. The upper curve (dotted) is a fit to data obtained requiring a 25 -mm-wide entrance cut. The lower curve (solid) is a fit to data obtained using a 5-mm-wide entrance cut. The "Percent" term represents the intrinsic sampling term ( $A$ in the text); a $5 \%$ photo-statistics stochastic term has been removed in the fit function.

into relatively large arrays is very attractive. These SiPMs are very fast and they can be mounted onboard, in the magnetic field, thus avoiding the costly and clumsy development of lightguides and external housing. We are adopting this as our baseline, given the time we have to develop this technique together with several of our new collaborators who have expertise in this field. 


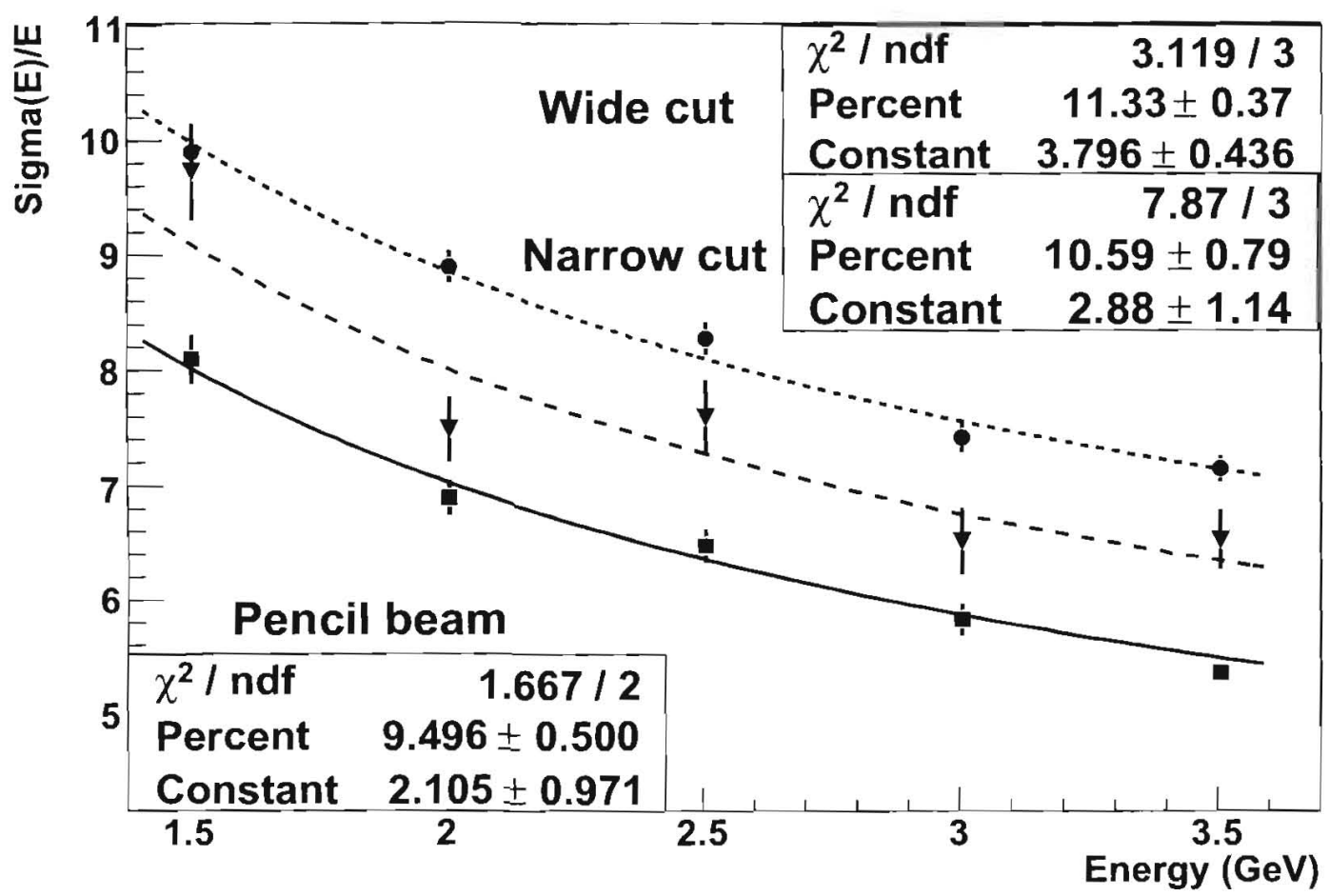

FIG. 48: Fits to resolution versus energy in the central module of an array of W/SciFi modules. Three entrance width cuts are imposed: $25 \mathrm{~mm}$ (dotted), $5 \mathrm{~mm}$ (dashed), and $1 \mathrm{~mm}$ "pencil" (solid). 


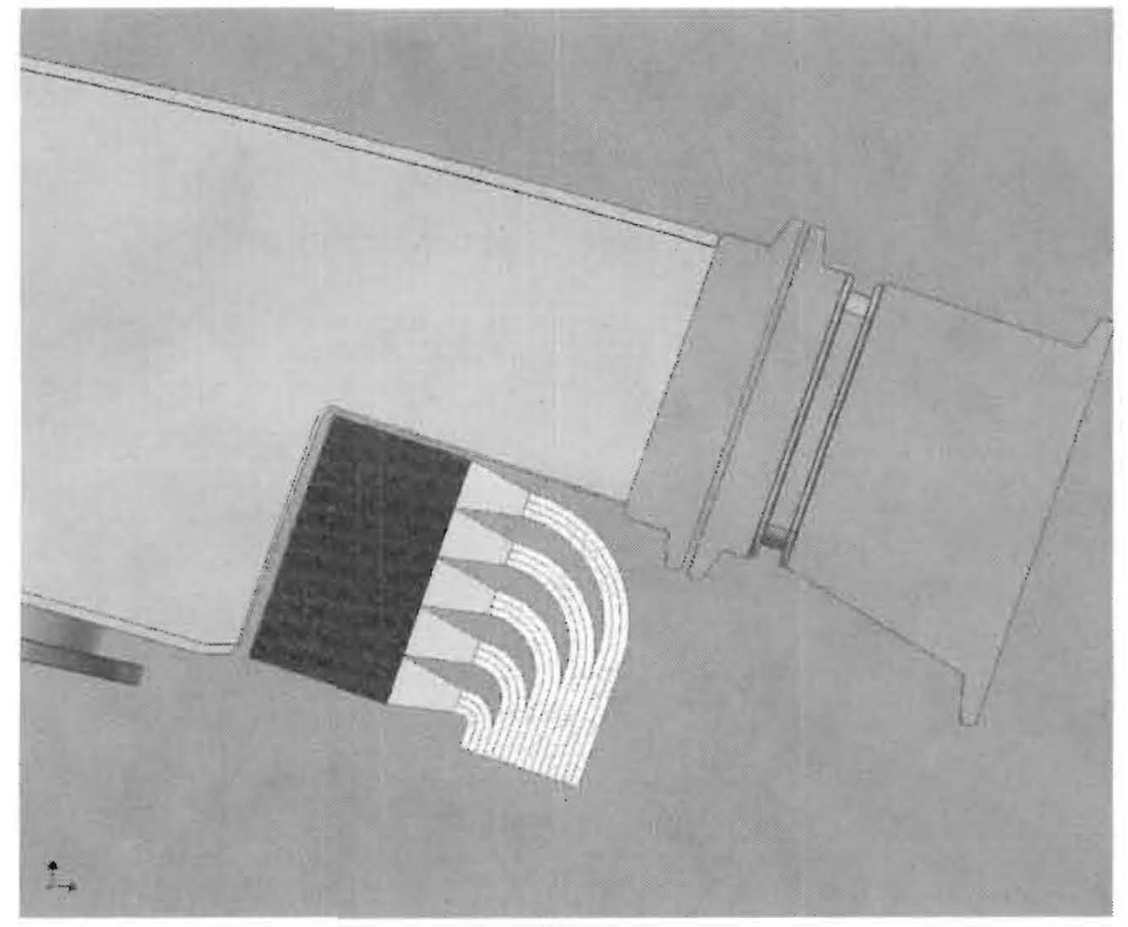

FIG. 49: Plan view of new calorimeter in the region of the bellows between vacuum chambers where the available space for lightguides is quite restricted. 